\title{
On deformability of atoms - comparative study between atoms and atomic nuclei
}

\author{
Tomoya Naito (内藤智也 $)^{1,2}$, Shimpei Endo (遠藤晋平 $)^{3,4}$, \\ Kouichi Hagino (萩野浩一) ${ }^{5}$, and Yusuke Tanimura (谷村雄 \\ 介 $)^{3,6}$ \\ ${ }^{1}$ Department of Physics, Graduate School of Science, The University of Tokyo, \\ Tokyo 113-0033, Japan \\ 2 RIKEN Nishina Center, Wako 351-0198, Japan \\ 3 Department of Physics, Tohoku University, Sendai 980-8578, Japan \\ ${ }^{4}$ Frontier Research Institute for Interdisciplinary Science, Tohoku University, \\ Sendai 980-8578, Japan \\ 5 Department of Physics, Kyoto University, Kyoto 606-8502, Japan \\ ${ }^{6}$ Graduate Program on Physics for the Universe, Tohoku University, Sendai \\ 980-8578, Japan \\ E-mail: tomoya.naito@phys.s.u-tokyo.ac.jp, \\ shimpei.endo@nucl.phys.tohoku.ac.jp, hagino.kouichi.5m@kyoto-u.ac.jp, \\ tanimura@nucl.phys.tohoku.ac.jp
}

\begin{abstract}
Atomic nuclei can be spontaneously deformed into non-spherical shapes as many-nucleon systems. We discuss to what extent a similar deformation takes place in many-electron systems. To this end, we employ several manybody methods, such as the unrestricted Hartree-Fock method, post-Hartree-Fock methods, and the density functional theory, to compute the electron density distribution in atoms. We show that the electron density distribution of openshell atoms is deformed due solely to the single-particle valence orbitals, while the core part remains spherical. This is in contrast to atomic nuclei, which can be deformed collectively. We qualitatively discuss the origin for this apparent difference between atoms and nuclei by estimating the energy change due to deformation. We find that nature of the interaction plays an essential role for the collective deformation.
\end{abstract}

Submitted to: J. Phys. B: At. Mol. Phys. 


\section{Introduction}

Atoms and atomic nuclei share common features as quantum many-body systems of interacting fermions [1]. In atoms, the inter-particle interaction is the repulsive Coulomb interaction between electrons, and there exists the spherical external Coulomb potential due to the nucleus. On the other hand, in atomic nuclei, the inter-particle interaction is the attractive nuclear force between two or more nucleons, with no external potential. Despite the differences in the fundamental interactions, many similar properties have been observed in both systems, such as the shell structure and the associated magic numbers [2, 3].

One of the most important properties of atomic nuclei is the collective nuclear deformation, which is evidenced by the characteristic rotational spectra [4, 5]. It was discussed that this collective deformation is originated from the strong attractive interaction between protons and neutrons [6, 7, 8, 9]. Here, atomic nuclei are deformed as a whole, resulting in a strong enhancement in the transition probability from the first excited state to the ground state. This is in marked contrast to trivial deformation seen in nuclei with a valence nucleon outside a doubly-magic nucleus [10, 11, 12, 13]. Since doubly-magic nuclei are, in general, spherical, such deformation is entirely due to the valence nucleon, thus of single-particle nature, unless the valence nucleon induces a strong polarization effect of the core nucleus [14. Although some of such atomic nuclei are known to be deformed beyond single-particle level, the collective deformation is known to be much more significant than such a singe-particle deformation. We note that atomic nuclei may also be deformed due to the $\alpha$-cluster formation [15, 16, 17, 18, 19, 20, 21, 22, 23, 24, 25, even though it is out of the scope of this paper. See [4, 5] for more details.

A natural question then arises: Can atoms be deformed collectively as in atomic nuclei? Notice that calculations of the atomic structure have been usually carried out by assuming spherical symmetry [26, 27, 28, and the non-sphericity has been taken care of only in a few works [29, 30. Likewise, spherical symmetry has been usually assumed to construct the pseudopotential for valence electrons in calculations of molecules and solids [31, 32, 33, 34]. It is thus widely believed that atoms are rather spherical, in stark contrast to deformed atomic nuclei. What is the physical origin of this difference? To what extent can electron density distributions in atoms be deformed collectively?

To answer these questions, in this paper we calculate electron density distributions of atoms and their deformation parameters using various quantum chemical methods without assuming spherical symmetry. Namely, we use and compare the unrestricted Hartree-Fock method [?, ?, ?, ?, 35, 36, 37, 38, 39], several post-Hartree-Fock methods [39, 40, 41, 42, 43, 44, 45, 46, 47, 48, 49, 50, 51, and the density functional theory (DFT) [52, 53, 54. By estimating the change in the energy induced by the deformation, in nuclear systems, we find that the spin-up and spin-down particles tend to be deformed in the same manner due to the attractive inter-particle nuclear interaction. In atomic systems, on the other hand, we find that the spin-up and spindown particles prefer to be deformed in the opposite manner, cancelling each other and resulting in a small deformation as a whole due to the repulsive inter-particle Coulomb interaction. We, thus, show that the difference between atoms and atomic nuclei in their deformability primarily originates from the different nature of the inter-particle interactions.

The paper is organized as follows. In section 2 , we first give a brief introduction of 
the deformation of many-body systems, and introduce a parameter $\beta$ to characterize the deformation. We then carry out quantum chemical calculations for isolated atoms and discuss their deformations in section 3 . In section 4, we qualitatively compare between atoms and nuclei using a model to estimate a change in the energy, and discuss the origin for collective deformation. We then summarize the paper in section 5 . In Appendix A, we discuss the deformation of neutron drops, that is, many-neutron systems trapped in a harmonic oscillator potential, and exemplify that nuclear systems can be deformed collectively. In Appendix B a generalization of the calculations in section 4 is presented. Appendix C summarizes the numerical data for atomic deformation calculated in the main part of the paper.

\section{Collective deformation of many-fermion systems}

\subsection{Deformation parameters}

Before we present the results of our calculations, let us briefly summarize the basic concepts of collective deformation. Atomic nuclei in the rare earth region, such as ${ }^{154} \mathrm{Sm}$ and ${ }^{168} \mathrm{Er}$, as well as those in the actinide region, such as ${ }^{234} \mathrm{U}$, often exhibit characteristic spectra, in which the energy of the state with the total angular momentum $I(I=0,2,4, \ldots)$ is proportional to $I(I+1)$. These spectra are interpreted as that those nuclei are deformed in the ground state and show the rotational spectra given by

$$
E_{\mathrm{rot}}=\frac{I(I+1) \hbar^{2}}{2 \mathcal{I}}
$$

where $\mathcal{I}$ is the moment of inertia for the rotational motion. An important fact is that a large number of nucleons in those nuclei are involved in the deformation, and thus such deformation is referred to as collective deformation. Indeed, the electromagnetic transition probability from e.g., the $I=2$ state to the $I=0$ state (i.e. the ground state) is significantly enhanced due to the collectivity of deformation [5].

The collective deformation is characterized by a non-zero value of the quadrupole moment $Q_{i j}$ defined by

$$
Q_{i j}=\int\left(3 r_{i} r_{j}-\delta_{i j} r^{2}\right) \rho(\boldsymbol{r}) d \boldsymbol{r} \quad(i, j=x, y, z),
$$

where $\rho(\boldsymbol{r})$ is the density distribution. Note that there exist several normalizations for the quadrupole moment tensor $Q$. Here, we take the normalization often used in nuclear physics [5], which is sometimes referred to as "traceless quadrupole moment" in the context of quantum chemistry.

The symmetric matrix $Q_{i j}$ can be diagonalized to define the cartesian axes, $x^{\prime}, y^{\prime}$, and $z^{\prime}$ in the intrinsic frame. With the eigenvalues of $Q_{i j}$, the deformation parameters $\beta_{k}$ can be defined as

$$
\beta_{k}=\sqrt{\frac{\pi}{5}} \frac{Q_{k}}{N_{\text {tot }}\left\langle r^{2}\right\rangle} \quad\left(k=x^{\prime}, y^{\prime}, z^{\prime}\right),
$$

where $Q_{k}$ is an eigenvalue of $Q_{i j}$ and $\left\langle r^{2}\right\rangle$ is the mean-square radius given by

$$
\left\langle r^{2}\right\rangle=\frac{\int r^{2} \rho(\boldsymbol{r}) d \boldsymbol{r}}{\int \rho(\boldsymbol{r}) d \boldsymbol{r}}=\frac{1}{N_{\text {tot }}} \int r^{2} \rho(\boldsymbol{r}) d \boldsymbol{r},
$$


with $N_{\text {tot }}$ being the total number of particles in the system. For atoms, $N_{\text {tot }}$ is the total number of electrons (the atomic number) $Z$, whereas for atomic nuclei it is the total number of nucleons (the mass number) $A$.

The deformation parameter $\beta_{j}$ is closely related to the angle-dependent radius of the system, $R(\theta, \phi)$. The radius can be expanded in multipoles with spherical harmonics $Y_{l m}$ as [5]

$$
R(\theta, \phi)=R_{0}\left[1+\sum_{l=2}^{\infty} \sum_{m=-l}^{l} a_{l m} Y_{l m}^{*}(\theta, \phi)\right] .
$$

The monopole part $(l=0)$ in the expansion is absorbed in the definition of $R_{0}$, while the dipole part $(l=1)$ need not be considered since, to the first order, it merely shifts the center of mass of the whole system without changing its shape. If one considers only the quadrupole deformation with $l=2$, after transforming to the intrinsic frame, (5) is reduced to

$$
\begin{aligned}
R\left(\theta^{\prime}, \phi^{\prime}\right) & =R_{0}\left[1+\beta \cos \gamma Y_{20}\left(\theta^{\prime}, \phi^{\prime}\right)+\frac{1}{\sqrt{2}} \beta \sin \gamma Y_{22}\left(\theta^{\prime}, \phi^{\prime}\right)+\frac{1}{\sqrt{2}} \beta \sin \gamma Y_{2-2}\left(\theta^{\prime}, \phi^{\prime}\right)\right] \\
& =R_{0}\left[1+\sqrt{\frac{5}{16 \pi}} \beta\left\{\cos \gamma\left(3 \cos ^{2} \theta^{\prime}-1\right)+\sqrt{3} \sin \gamma \sin ^{2} \theta^{\prime} \cos 2 \phi^{\prime}\right\}\right]
\end{aligned}
$$

with $a_{20}^{\prime}=\beta \cos \gamma, a_{22}^{\prime}=a_{2-2}^{\prime}=\frac{1}{\sqrt{2}} \beta \sin \gamma$, and $a_{21}^{\prime}=a_{2-1}^{\prime}=0$, where the primes denote the quantities in the intrinsic frame. Here, $\beta>0$ and $\gamma$ represent the degrees of elongation and triaxiality [5]. Due to the symmetry, $\gamma$ can be restricted to $\gamma \in[0, \pi / 3]$. In addition, $\gamma=\pi / 3$ with $\beta>0$ is identical to $\gamma=0$ with $\beta<0$. Hence, if one only considers the axially symmetric case with both positive and negative $\beta,(6)$ can be simplified further as

$$
R\left(\theta^{\prime}, \phi^{\prime}\right)=R_{0}\left[1+\sqrt{\frac{5}{16 \pi}} \beta\left(3 \cos ^{2} \theta^{\prime}-1\right)\right],
$$

with axially symmetric shape along the $z^{\prime}$ axis. In this case, the radii in the $x^{\prime}, y^{\prime}$, and $z^{\prime}$ directions are given by

$$
\begin{gathered}
R_{x^{\prime}}=R_{y^{\prime}}=R\left(\pi / 2, \phi^{\prime}\right)=R_{0}\left(1-\sqrt{\frac{5}{16 \pi}} \beta\right), \\
R_{z^{\prime}}=R\left(0, \phi^{\prime}\right)=R_{0}\left(1+2 \sqrt{\frac{5}{16 \pi}} \beta\right),
\end{gathered}
$$

respectively. Thus, the density distribution is spherical for $\beta=0$, while for $\beta>0$ and $\beta<0$ it becomes prolate and oblate shapes, respectively, as shown in figure 1 . For a positive value of $\beta$, the $z^{\prime}$ axis makes the longest axis as shown schematically in figure 2,

The quadrupole deformation parameter $\beta$ here corresponds to $\beta_{z}$ defined by (3) (to the leading order, at least for a sharp-cut density distribution with the radius $R(\theta, \phi)[5])$. Note that the condition of $Q_{x}=Q_{y}=-Q_{z} / 2$, i.e. $\beta_{x}=\beta_{y}=-\beta_{z} / 2=$ $-\beta / 2$, holds for the axially symmetric case. 


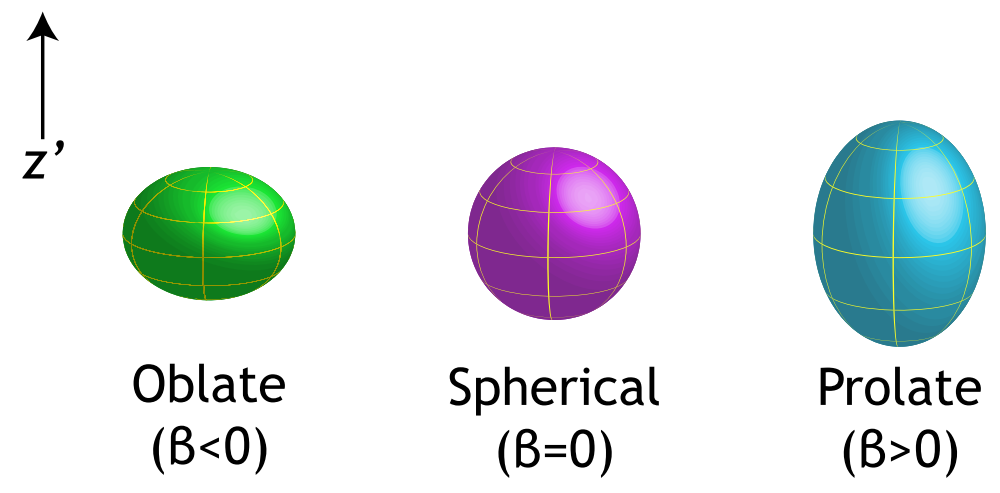

Figure 1. A schematic picture of the collective deformation.

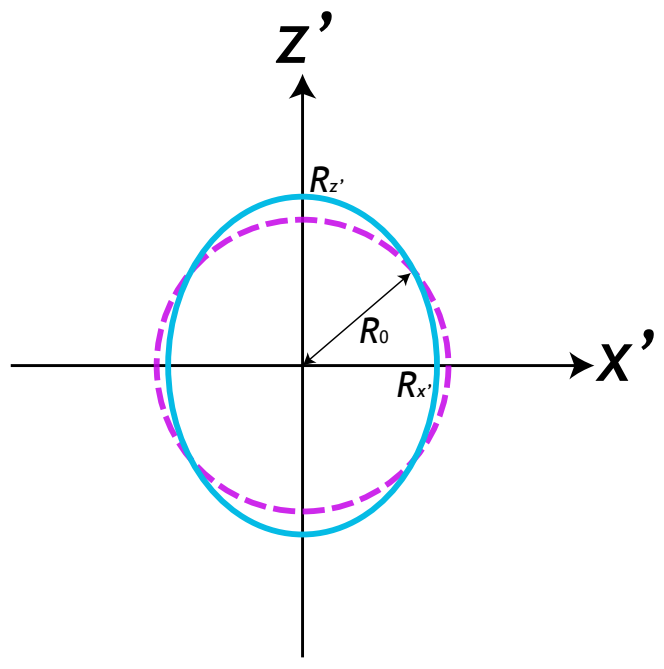

Figure 2. A schematic picture of a collective deformation in the $x^{\prime} z^{\prime}$ plane defined in the intrinsic frame. The axially symmetric shape along the $z^{\prime}$ axis is assumed. The dashed and the solid curves correspond to the deformation parameter of $\beta=0$ and 0.25 , respectively.

If the particles in different spin states undergo different deformations, as in the case for the electrons in atoms to be discussed in section 4 , spin-dependent deformation parameters $\beta_{\uparrow}$ and $\beta_{\downarrow}$ can be also defined. Since $\rho(\boldsymbol{r})=\rho_{\uparrow}(\boldsymbol{r})+\rho_{\downarrow}(\boldsymbol{r})$ holds, $Q=Q_{\uparrow}+Q_{\downarrow}$ also does, where $\rho_{\uparrow}$ and $\rho_{\downarrow}$ denote the densities of up-spin and down-spin fermions, respectively.

It should also be noted that the discussion here is based on the intrinsic frame. In the laboratory frame, there is no criterion to choose the symmetry axis (that is, the $z$ axis) and the system would show spherical distribution, even when it is deformed in the intrinsic frame.

Microscopically, the collective deformation discussed in this section is intimately related to the mean-field theory. In this theory, particles move independently in a mean field potential, which is formed self-consistently by the interaction among the particles. The collective deformation occurs as a consequence of spontaneous breaking 
of rotational symmetry. That is, a system may be intrinsically deformed and breaks the rotational symmetry even if the total Hamiltonian has the symmetry. This is actually a good advantage of the self-consistent mean-field theory used in section 3 . since a large part of the many-body correlations can be taken into account while keeping the simple picture of independent particles [5, 55, 56, 57.

\subsection{Single-particle contribution to deformation}

In connection to the deformation in atoms discussed in the next section, it is instructive to compute the deformation parameters discussed in the previous subsection for a system with a spherical core plus a valence particle. In this case, the quadrupole moment of the system entirely comes from the valence particle, since 2 vanishes for the spherical density distribution. If one assumes that the wave function for the valence particle is given by

$$
\psi_{n l m s}(\boldsymbol{r})=\frac{u_{n l}(r)}{r} Y_{l m}(\theta, \phi) \chi_{s},
$$

as a single-particle orbital in a spherical (effective) potential, where $\chi_{s}$ is the spinor of the valence particle, the quadrupole moments of the whole system read $Q_{i j}=q_{i j}^{\text {(sp) }}$ with

$$
\begin{aligned}
q_{i j}^{(\mathrm{sp})} & =\int\left(3 r_{i} r_{j}-\delta_{i j} r^{2}\right)\left|\psi_{n l m s}(\boldsymbol{r})\right|^{2} d \boldsymbol{r} \\
& =\int\left(3 \mathfrak{s}_{i} \mathfrak{s}_{j}-\delta_{i j}\right) r^{2}\left|\psi_{n l m s}(\boldsymbol{r})\right|^{2} d \boldsymbol{r} .
\end{aligned}
$$

Here, we have introduced the notation $r_{i}=r \mathfrak{s}_{i}$ with

$$
\mathfrak{s}_{i}= \begin{cases}\sin \theta \cos \phi & (i=x), \\ \sin \theta \sin \phi & (i=y), \\ \cos \theta & (i=z) .\end{cases}
$$

Substituting 10 , one finds

$$
q_{i j}^{(\mathrm{sp})}=\int_{0}^{\infty} r^{2}\left|u_{n l}(r)\right|^{2} d r \int\left(3 \mathfrak{s}_{i} \mathfrak{s}_{j}-\delta_{i j}\right)\left|Y_{l m}(\theta, \phi)\right|^{2} d \Omega,
$$

where $d \Omega$ is the angular part of $d \boldsymbol{r}$. Note that the off-diagonal components of $q_{i j}^{(\mathrm{sp})}$ vanish. The deformation parameter for the valence particle, that is, the single-particle deformation parameter, then reads,

$$
\beta_{i}^{(\mathrm{sp})}=\sqrt{\frac{\pi}{5}} \frac{q_{i i}^{(\mathrm{sp})}}{\left\langle r^{2}\right\rangle_{n l}}=\sqrt{\frac{\pi}{5}} \int\left(3 \mathfrak{s}_{i}^{2}-1\right)\left|Y_{l m}(\theta, \phi)\right|^{2} d \Omega .
$$

Notice that the radial integral in 13 is cancelled with the mean-square radius

$$
\left\langle r^{2}\right\rangle_{n l}=\int_{0}^{\infty} r^{2}\left|u_{n l}(r)\right|^{2} d r
$$

The deformation parameter of the whole system (i.e. the core plus the valence particle) is then given by

$$
\beta_{i}=\sqrt{\frac{\pi}{5}} \frac{q_{i}^{(\mathrm{sp})}}{N_{\mathrm{tot}}\left\langle r^{2}\right\rangle}
$$


where $\left\langle r^{2}\right\rangle=\left[\left(N_{\text {tot }}-1\right)\left\langle r^{2}\right\rangle_{\text {core }}+\left\langle r^{2}\right\rangle_{n l}\right] / N_{\text {tot }}$ is the mean-square radius of the whole system with $\left\langle r^{2}\right\rangle_{\text {core }}$ being that of the core. If one assumes the radius of the whole system is similar to the radius of the valence orbital, $\left\langle r^{2}\right\rangle \simeq\left\langle r^{2}\right\rangle_{n l}$, which is reasonable in the case of atomic nuclei, the deformation parameter is given by

$$
\beta_{i} \simeq \frac{\beta_{i}^{(\mathrm{sp})}}{N_{\mathrm{tot}}} .
$$

In contrast, in the case of atoms, valence electrons have a larger spatial distribution and contribute more significantly than inner core electrons to the mean-square radius $\left\langle r^{2}\right\rangle$. If one assumes $\left\langle r^{2}\right\rangle \simeq\left\langle r^{2}\right\rangle_{n l} / N_{\text {tot }}$, i.e. only one electron mainly contributes to $\left\langle r^{2}\right\rangle$, one obtains

$$
\beta_{i} \simeq \beta_{i}^{(\mathrm{sp})} .
$$

Notice that when the number of valence electrons is $N_{\text {val }}$, the deformation parameter is, instead, approximately given by

$$
\beta_{i} \simeq \frac{\sum_{\mathrm{val}} \beta_{i}^{(\mathrm{sp})}}{N_{\mathrm{val}}} .
$$

Let us evaluate explicitly the single-particle deformation parameter for the pure $p$ and $d$ orbitals. To this end, we define the indices of the real spherical harmonics as follows [58]:

$$
\begin{aligned}
Y_{p}^{x}(\theta, \phi) & =\sqrt{\frac{3}{4 \pi}} \sin \theta \cos \phi, \\
Y_{p}^{y}(\theta, \phi) & =\sqrt{\frac{3}{4 \pi}} \sin \theta \sin \phi, \\
Y_{p}^{z}(\theta, \phi) & =\sqrt{\frac{3}{4 \pi}} \cos \theta,
\end{aligned}
$$

and

$$
\begin{aligned}
Y_{d}^{x y}(\theta, \phi) & =\sqrt{\frac{15}{16 \pi}} \sin ^{2} \theta \sin 2 \phi, \\
Y_{d}^{y z}(\theta, \phi) & =\sqrt{\frac{15}{16 \pi}} \sin 2 \theta \sin \phi, \\
Y_{d}^{z x}(\theta, \phi) & =\sqrt{\frac{15}{16 \pi}} \sin 2 \theta \cos \phi, \\
Y_{d}^{x^{2}-y^{2}}(\theta, \phi) & =\sqrt{\frac{15}{16 \pi}} \sin ^{2} \theta \cos 2 \phi, \\
Y_{d}^{z^{2}}(\theta, \phi) & =\sqrt{\frac{5}{16 \pi}}\left(3 \cos ^{2} \theta-1\right) .
\end{aligned}
$$

Using these notations, the deformation parameter $\beta_{i}^{(\mathrm{sp})}$ for $Y_{p}^{j}$ is found to be

$$
\beta_{i}^{(\mathrm{sp})}= \begin{cases}\frac{4}{5} \sqrt{\frac{\pi}{5}}=0.6341 & (i=j), \\ -\frac{2}{5} \sqrt{\frac{\pi}{5}}=-0.3171 & \text { (Otherwise) } .\end{cases}
$$


For the $d$-orbitals, the deformation parameter for $Y_{d}^{j k}((j, k)=(x, y),(y, z),(z, x))$ is given by

$$
\beta_{i}^{(\mathrm{sp})}= \begin{cases}-\frac{4}{7} \sqrt{\frac{\pi}{5}}=-0.4530 & (i \neq j \text { and } i \neq k), \\ \frac{2}{7} \sqrt{\frac{\pi}{5}}=0.2265 & \text { (Otherwise) }\end{cases}
$$

while the deformation parameters for $Y_{d}^{x^{2}-y^{2}}$ and $Y_{d}^{z^{2}}$ read

$$
\beta_{i}^{(\mathrm{sp})}= \begin{cases}-\frac{4}{7} \sqrt{\frac{\pi}{5}}=-0.4530 & (i=z), \\ \frac{2}{7} \sqrt{\frac{\pi}{5}}=0.2265 & \text { (Otherwise) }\end{cases}
$$

and

$$
\beta_{i}^{(\mathrm{sp})}= \begin{cases}\frac{4}{7} \sqrt{\frac{\pi}{5}}=0.4530 & (i=z), \\ -\frac{2}{7} \sqrt{\frac{\pi}{5}}=-0.2265 & \text { (Otherwise) },\end{cases}
$$

respectively. Note that due to higher-order deformations, the $d$ orbitals are not axially symmetric except the $d_{z^{2}}$ orbital, even though triaxiality vanishes $(\gamma=0)$.

\section{Quantum Chemical Calculation}

In this section, a possibility of the deformation of isolated atoms is studied numerically with the computational code GAUSSIAN [59]. In the GaUSSIAN code, the wave function and the energy of the ground state of atomic and molecular systems are numerically calculated by using the Gaussian-type basis expansion. With this code, all-electron calculation can be performed for a wide range of atoms $(Z \leq 54)$.

First, our calculation setup is explained in section 3.1. Then, the results and their basic explanations are presented in sections 3.2 and 3.3 where we shall see that open-shell atoms have non-zero deformation parameters. To understand clearly the mechanism of such deformations, we discuss the results in more details in section 3.4 by analyzing the deformations of each single-particle orbital.

For convenience, the Hartree atomic unit $m_{e}=\hbar=e^{2}=a_{\mathrm{B}}=4 \pi \varepsilon_{0}=1$ is used, where $m_{e}$ is the mass of electrons, $\varepsilon_{0}$ is the permittivity of vacuum, and $a_{\mathrm{B}}=5.2918 \ldots \times 10^{-11} \mathrm{~m}$ is the Bohr radius 60, 61]. The unity of the electric quadrupole moment in the Hartree atomic unit corresponds to $4.4866 \ldots \times$ $10^{-40} \mathrm{C} \mathrm{m}^{2}=1.3450 \ldots$ Debye $\AA$. Here, we use quadrupole moment of density itself, instead of charge density, and thus the electric quadrupole moment corresponds to $-e$ $(<0)$ times of $Q_{k}$ in this paper.

\subsection{Calculation setup and deformation parameters}

An isolated neutral atom with the atomic number $Z$ in the non-relativistic scheme is described by the Hamiltonian

$$
H=-\frac{1}{2} \sum_{j=1}^{Z} \Delta_{j}+\sum_{j=1}^{Z} V_{\mathrm{ext}}\left(\boldsymbol{r}_{j}\right)+\sum_{1 \leq j<k \leq Z} V_{\mathrm{int}}\left(\boldsymbol{r}_{j}, \boldsymbol{r}_{k}\right),
$$


where $\boldsymbol{r}_{j}$ is the coordinate of the particle $j$. The external potential $V_{\text {ext }}$ and the electron-electron interaction $V_{\text {int }}$ read

$$
\begin{aligned}
V_{\mathrm{ext}}(\boldsymbol{r}) & =-\frac{Z}{r}, \\
V_{\mathrm{int}}\left(\boldsymbol{r}_{j}, \boldsymbol{r}_{k}\right) & =\frac{1}{r_{j k}},
\end{aligned}
$$

respectively, where $r=|\boldsymbol{r}|$ and $r_{j k}=\left|\boldsymbol{r}_{j}-\boldsymbol{r}_{k}\right|$. Here, the atomic nucleus is assumed to be a point charge with infinitely heavy mass, and the spin-orbit interaction is neglected.

In this paper, the many-body Schrödinger equation is approximately solved using mainly the unrestricted Hartree-Fock (UHF) method [35, 36, 37, 38, 39. The quadrupole deformation parameters of atoms have not yet been directly observed with sufficient accuracy to compare with our theoretical results. Moreover, it is generally known to be difficult to systematically estimate theoretical errors in quantum chemical calculations. For this reason, we regard the difference among the results of various methods and bases as crude estimates of the error in the quadrupole moment $Q_{i}$ and the correspoinding deformation parameter $\beta$.

To assess the dependence of the many-body methods, several post-HartreeFock methods and the DFT [52, 53, 54] with the PZ81 local density approximation (LDA) exchange-correlation functional 62] are also employed. As for the postHartree-Fock methods, we choose the configuration interaction [44, 45, 47, 51] with double excitations (CID) and single-double excitations (CISD), the coupled-cluster method [41, 42, 43, with double excitations (CCD) and single-double excitations (CCSD), and Møller-Plesset many-body perturbation theory [40] with second (MP2), third (MP3), and fourth (MP4) orders [46, 49, 48, 50]. For the MP4 calculation, the excitations are restricted to single, double, and quadruple excitations (MP4SDQ), which is simply referred to as MP4. The calculations are performed with these post Hartree-Fock methods, i.e. CID, CISD, CCD, CCSD, MP2, MP3, and MP4, based on the UHF calculation. In these post Hartree-Fock calculations, the core is not treated as frozen.

To perform the many-body calculations, the basis expansions with the 631+G 63, 64, dAug-CC-pV5Z [65, 66], and STO-3G 67] bases are used. It should be noted that the different bases are optimized to different ranges of atoms [59]: The STO-3G basis can be applied from $\mathrm{H}$ atom to Xe atom. The applicable ranges of the 6-31+G and the dAug-CC-pV5Z bases are limited to atoms from $\mathrm{H}$ to $\mathrm{Kr}$. In addition, the dAug-CC-pV5Z basis cannot be applied to $\mathrm{Mg}, \mathrm{K}$, or $\mathrm{Ca}$ atoms. In this paper, we mainly employ the $6-31+\mathrm{G}$ basis, while the results with the dAug-CC-pV5Z and STO-3G bases are also shown in Appendix C.

To perform the calculation with the GAUSSIAN code, the multiplicity of the ground states has to be assumed, which are taken from the database provided by the National Institute of Standards and Technology (NIST) 68. We do not assume any symmetry for the initial condition, nor use the filling approximation, in which valence orbitals in open shells are filled with equal occupation probabilities I With this procedure, non-spherical electron density can be described properly. After the

$\ddagger$ For example, in the case of $\mathrm{B}, 2 p_{x}, 2 p_{y}$, and $2 p_{z}$ orbitals are occupied with the probability of $1 / 3$. Notice that the wave function in the filling approximation is different from the coherent superposition of those orbitals, $\mid$ val $\rangle=\left(\left|2 p_{x}\right\rangle+\left|2 p_{y}\right\rangle+\left|2 p_{z}\right\rangle\right) / \sqrt{3}$, even though both of them lead to a spherical density distribution. 
calculation is converged, the valence-electron configurations are evaluated by using the natural orbital analysis [69, 70, 71, 72].

After we obtain the electron density $\rho(\boldsymbol{r})$ with these quantum chemical methods, the mean-square radius $\left\langle r^{2}\right\rangle$ and the quadrupole moments $Q_{i}$ are calculated according to (2) and (4), respectively. Here, we define the coordinate frame so that the quadrupole moment tensor $Q$ is diagonalized (see (3)).

\subsection{Systematic behaviors of atomic deformations and their basis dependence}

Let us now numerically investigate deformation in atoms. We first show in figure 3 the deformation parameter $\beta$ for atoms from $\operatorname{Li}(Z=3)$ to $\operatorname{Kr}(Z=36)$ calculated with the unrestricted Hartree-Fock method with the $6-31+\mathrm{G}$ basis. See Table C1 in Appendix $\mathrm{C}$ for the actual values. In figure 3 , we use different symbols to classify the nature of the atoms: The filled circles show noble-gas (i.e. closed-shell) atoms. The filled squares show half-closed-shell atoms; atoms with three valence electrons occupying the outer-most $p$ orbitals, or with five valence electrons occupying the outer-most $d$ orbitals. The triangles, the inverse triangles, and the diamonds show the others; atoms with $s, p$, and $d$ orbitals for the outer-most open shell, respectively [73]. Note that the octupole moments for all the calculated atoms are found to be zero.

Spherical atoms: According to figure 3, all the noble-gas atoms are spherical $(\beta=0)$, as is expected. Such trivial results are guaranteed by the fact that all the orbitals for any given principal and azimuthal quantum numbers, $n$ and $l$, are either fully occupied or unoccupied, and thus their distributions are, in total, spherical. In other words, $\sum_{m=-l}^{l}\left|Y_{l m}(\theta, \phi)\right|^{2}=(2 l+1) / 4 \pi$ is a constant, independent of the angles $\theta$ and $\phi$.

For the other atoms, the electronic configuration of their cores is the same as that of the noble-gas atoms. Hence, as long as the core density is not deformed due to non-trivial many-body effects, i.e. the polarization due to the interaction between the core and valence electrons, the deformation of the atom is expected to come only from the valence electrons. Possibility of such non-trivial deformation will be studied in details in section 3.4. but we can easily conclude before going to such details that the $s$-block and half-closed-shell atoms should be spherical $(\beta=0)$, as shown in figure 3 . The valence electrons of the $s$-block atoms by definition only occupy an $s$ orbital, and the many-body effects between the valence electrons and the spherically symmetric core necessarily results in a spherical electron density distribution. This is the case also for the half-closed-shell atoms: Atom with three electrons occupying valence $p$ orbitals, or those with five electrons occupying valence $d$ orbitals, are spherical in both cases, since $\alpha$-spin electrons occupy all the $(2 l+1)$ states with $m=-l,-l+1, \ldots, l$ due to the Hund rule [74, 75, 76, 77, 78.

Open-shell atoms: For the other open-shell atoms ( $p$-block and $d$-block atoms), the deformation parameters $\beta$ are generally non-zero. The deformation parameters for the atoms with the same group show similar tendencies, i.e. $|\beta| \simeq 0.1-0.3$ for the $p$-block atoms and $|\beta| \lesssim 0.01$ for the $d$-block atoms. This shows that the nature of the few valence open-shell electrons plays a major role in determining $\beta$ of the whole atom. In the context of nuclear physics, an atomic nucleus with the deformation parameter of $\simeq \pm 0.1$ or larger is regarded as a deformed nucleus (see Appendix A). It is thus tempting to regard the atoms with $|\beta| \gtrsim 0.1$ as significantly deformed as nuclei.

However, this deformation originates from single-particle valence orbitals and thus it is misleading to regard it as collective deformation. For example, the deformation of the 
$p$-block atoms can be understood as the valence $p$ orbitals mainly contributing to the deformation. Indeed, $|\beta| \simeq 0.1-0.3$ of the $p$-block atoms in figure 3 are similar values in order of magnitude as that of a single $p$ orbital given by $\sqrt{18}$ ) and $(22)$. On the other hand, the deformations of the $d$-block atoms are much smaller than those of the $p$-block atoms (see and compare with (23)). This implies that one cannot regard the deformation of $d$-block atoms as originating only from the single-orbital deformation. The mechanism of the deformation and the physical origin of this difference will be discussed in section 3.4. We will conclude there that the $p$-block atoms can be regarded as an almost inert core plus valence $p$-electrons, while many-body effects between the core and the valence electrons are relevant in the $d$-block atoms. In the $d$-block atoms, the core electrons tend to collectively cancel the deformation of the valence $d$ orbitals.

Exceptionally $\beta=0$ open-shell atoms are $\mathrm{V}(Z=23)$, Co $(Z=27), \mathrm{Cu}(Z=29)$, and $\mathrm{Zn}(Z=30)$. In the case of $\mathrm{Cu}$ and $\mathrm{Zn}$ atoms, ten of the valence electrons completely occupy the valence $d$ orbital and the remaining one or two valence electrons occupy the $s$ orbital. Thus, they are trivially spherical, in the same manner as the $s$ block atoms. In the case of $\mathrm{V}$ and Co atoms, on the other hand, three or eight valence electrons occupy the $d$ orbitals. At first sight, such open-shell $d$-block atoms do not seem to be trivially $\beta=0$. However, we note that they can be $\beta=0$ if $d_{x^{2}-y^{2}}$ and $d_{z^{2}}$ orbitals are occupied (unoccupied) at the same time $\S$. For example, $d^{8}$ occupation may lead to $\beta=0$ if the unoccupied orbitals are $d_{x^{2}-y^{2}}$ and $d_{z^{2}}$ orbitals. This is the case for Co atom. For $\mathrm{V}$ atom, $d^{3}$ occupation leads to $\beta=0$ because $d_{x^{2}-y^{2}}$ and $d_{z^{2}}$ orbitals are occupied simultaneously.

Basis dependence: In order to guarantee that our results presented in this section and in figure 3 do not depend on a specific choice of the basis in the calculation, we repeat calculations with different basis sets: In addition to the $6-31+\mathrm{G}$ basis used in figure 3 and Table C1 in Appendix C, we also use the dAug-CC-pV5Z and STO-3G bases. The results are shown in Table $\mathrm{C} 2$ and Table $\mathrm{C} 3$ in Appendix C respectively. We find that as long as the configurations of the valence electrons are the same, the deformation parameters $\beta$ are essentially the same, hence basis independent. We note, however, that there are a few results with the STO-3G basis which are different from those with the dAug-CC-pV5Z and 6-31+G bases, even when the configurations are the same. This may be because the STO-3G basis set spans a smaller space than the other basis sets, and thus the tail region of the electron density distribution $\rho(\boldsymbol{r})$ is not accurately described compared with the others.

\subsection{Many-body method dependence}

Our results presented in the previous section essentially remain the same, even if we employ other many-body methods than the unrestricted Hartree-Fock (UHF) method. As benchmark examples, $\mathrm{Al}(Z=13), \mathrm{Cu}(Z=29)$, and $\mathrm{Ga}(Z=31)$ are selected. In figure 4 , we show the deformation parameters $\beta$ for those atoms calculated with a wide variety of different many-body methods with the $6-31+\mathrm{G}$ basis. The data are shown in Table C4 in Appendix C. We find that the deformation parameters $\beta$ calculated with the post-Hartree-Fock methods (CCD, CCSD, CID, CISD, MP2, MP3, and MP4) are almost the same as those with the UHF. The excellent agreement of the UHF and the post-Hartree-Fock methods in figure 4 implies that the correlations beyond Hartree-Fock may be irrelevant to the quadrupole deformation.

$\S$ Higher-order deformations, such as hexadecapole deformation, exist even though they are considerably small. 

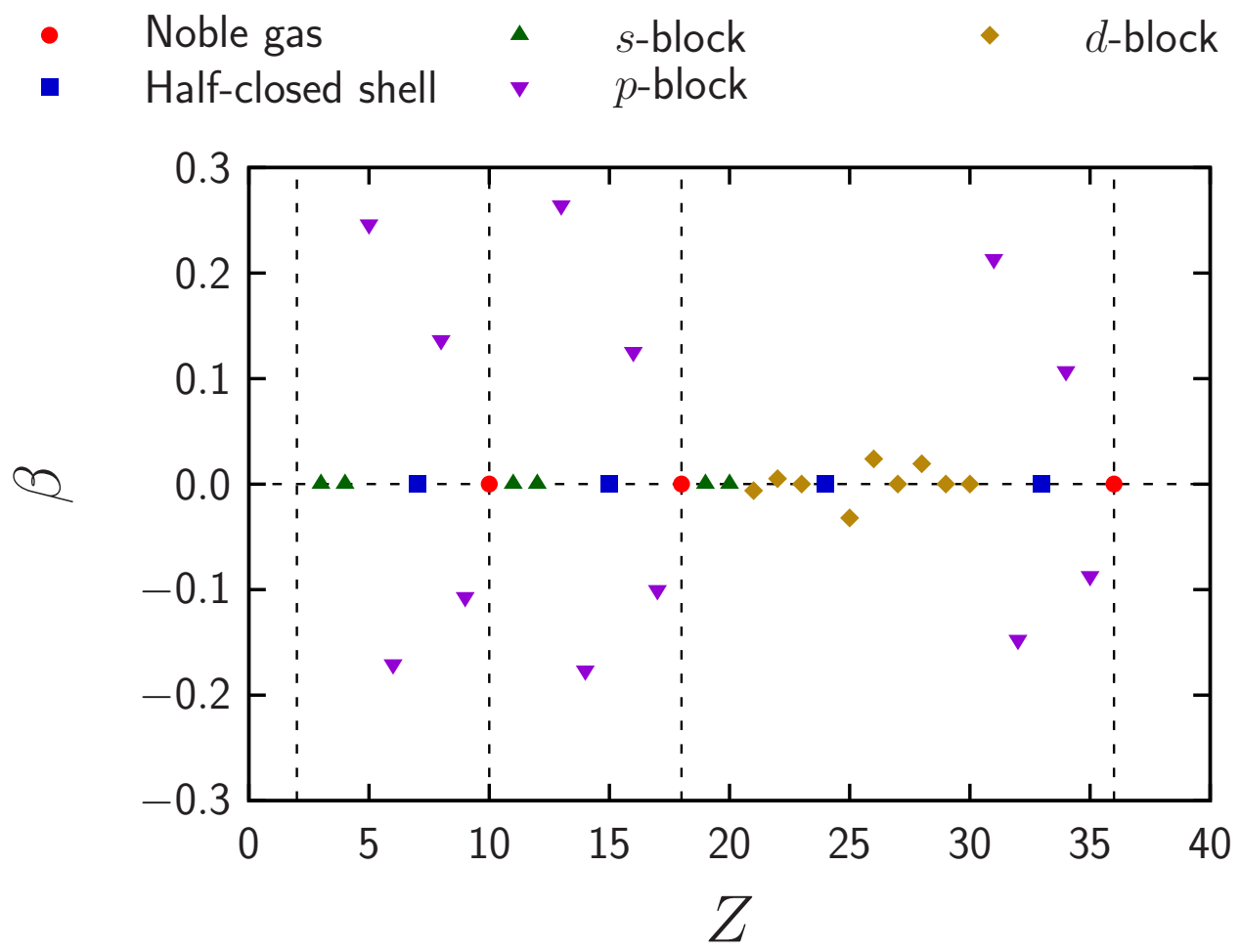

Figure 3. Deformation parameter $\beta$ as a function of the atomic number $Z$ from $\mathrm{Li}(Z=3)$ to $\mathrm{Kr}(Z=36)$ calculated with the unrestricted Hartree-Fock method with the $6-31+\mathrm{G}$ basis. The filled circles and the filled squares show noble-gas and half-closed-shell atoms, respectively. The triangles, the inverse-triangles, and the diamonds show the others; atoms with the outer-most open-shell $s$ orbitals ( $s$ block), $p$ orbitals ( $p$ block), and $d$ orbitals ( $d$ block), respectively. The vertical dashed lines denote the closed-shell atoms.

We have also performed the DFT calculation (indicated by LDA in figure 4) and found that the result also agrees excellently with the UHF and post-Hartree-Fock methods. Since the DFT method is not directly linked to the UHF calculation, there is no a priori reason why $\beta$ should be the same. In principle, owing to the HohenbergKohn theorem, the DFT calculation provides the exact, i.e. the full CI, density, if the exact exchange-correlation functional were known. In practice, the DFT results should depend on the choice of the employed exchange-correlation functional, since only approximated functional have been known. The excellent agreement between the DFT (LDA) and the other methods in figure 4, therefore, guarantees that finite deformation parameters presented in the previous section and following sections are not at all artifacts of a specific many-body approximation method.

We note that all the above results and discussions in this section hold true for the other bases: We have performed the same calculation also with dAug-CC-pV5Z basis as shown in Table C5 in Appendix C and obtained essentially the same results as those with the $6-31+\mathrm{G}$ basis in figure 4 and Table $\mathrm{C} 4$ 
On deformability of atoms

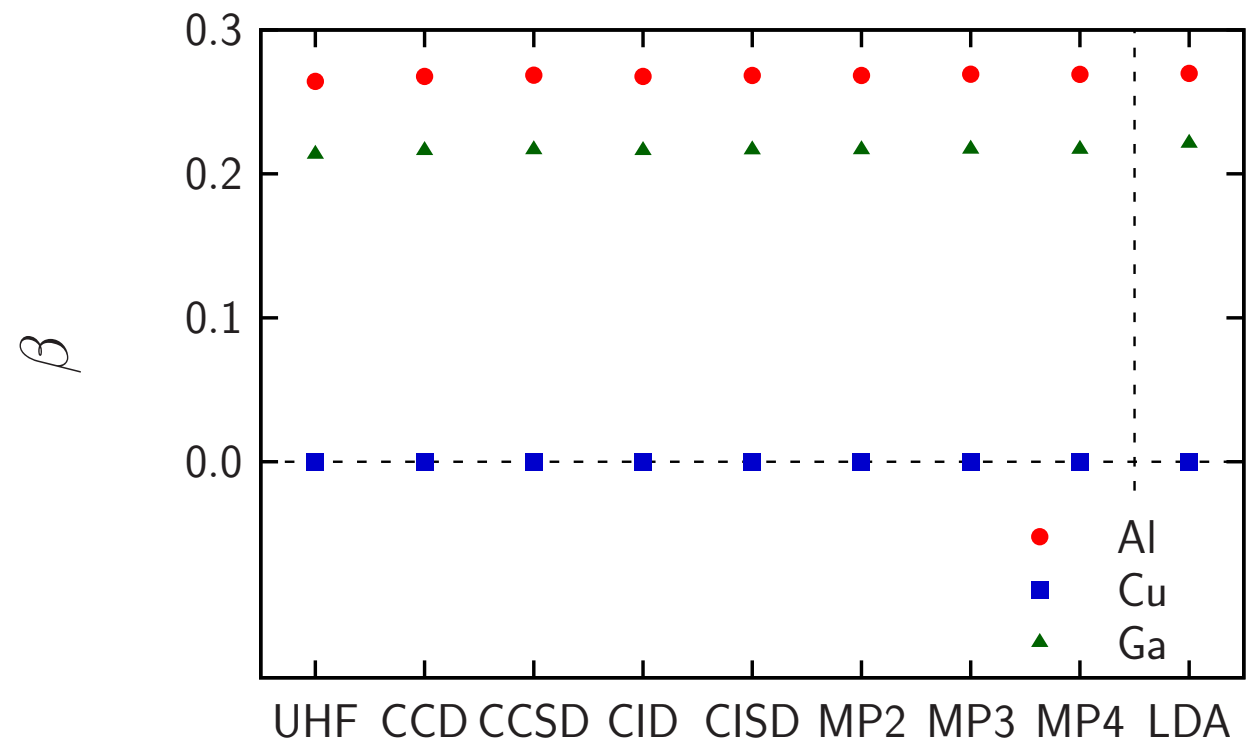

Figure 4. Dependence of the deformation parameter $\beta$ on many-body methods with the $6-31+\mathrm{G}$ basis. Data for $\mathrm{Al}(Z=13), \mathrm{Cu}(Z=29)$, and $\mathrm{Ga}(Z=31)$ are shown with circles, squares, and triangles, respectively. For more details, see the text.

\subsection{Analyses with single-particle orbitals}

To understand further the physical mechanism of the atomic deformations, let us define the deformation parameter of each single-particle orbital $\beta_{\max }^{(\mathrm{sp})}$ by $\beta_{i}^{(\mathrm{sp})}$ for the direction of the symmetry axis of the orbital. Here, the symmetry axis of the orbital may be different from that of the whole atoms, i.e. the $i$ axis for $p_{i}$ orbital, the $k$ axis for $d_{i j}$ orbital $(i \neq j \neq k)$, and the $z$ axis for the other $d$ orbitals. Angular-momentum mixing can occur due to the deformation, so that single-particle orbitals in these methods can, in general, show $\beta$ different from those of the pure spherical harmonics. Specifically, as we will see, an $s$ orbital can show a non-zero $\beta$ due to the mixture with $d$ orbitals. Note that $\beta_{\max }^{(\mathrm{sp})}$ is the deformation parameter for each single-particle orbital (see (14)), which is divided by the radius of each orbital. Therefore, $\beta_{i}$ of the whole atom is not a simple sum of $\beta_{\max }^{(\mathrm{sp})}$. Thus, a care must be taken when using them, but they are still useful in understanding the mechanism of the deformation microscopically.

Deformation of single-particle orbitals: Table 1 shows $\beta_{\max }^{(\mathrm{sp})}$ for selected valence orbitals of some open-shell atoms. All the data are calculated by the unrestricted Hartree-Fock calculation with the $6-31+\mathrm{G}$ basis. First, we find that the deformation parameters of the valence $p$ and $d$ orbitals are almost the same as those of the pure $p$ and $d$ orbitals given by (22)-(23c). This suggests that even when the atom is deformed, the single-particle orbitals of the valence $p$ and $d$ electrons are described almost as a product of the radial function and the pure spherical harmonics. In other words, the valence $p$ and $d$ electrons feel an almost perfectly spherical effective (Hartree-Fock or Kohn-Sham) potential. We can, therefore, conclude that the density 
of the electrons is almost perfectly spherical in the central region due to the strong Coulomb interaction of the nucleus, resulting in the spherical effective potential for the valence electrons. The electron density is deformed only in the surface and the tail regions of the atoms described by the valence electrons.

Deformations in the surface and degeneracy lifting: Such deformations lead to a non-spherical effective potential in the surface and the tail regions. There are several evidences for this. Firstly, the outer-most $s$ orbital can have non-zero $\beta$ and become non-spherical. This is shown in Table 1 as a non-zero $\beta$ of the $4 s$ orbitals, which tend to cancel the deformation of the valence $3 d$ orbitals. This is due to the screening effect. That is, the outer-most $4 s$ electrons try to cancel the deformed electron density of the open-shell valence $3 d$ electrons. We will discuss this $s$-orbital deformation in more details later in this section taking Sc atom as an example.

Secondly, the orbitals with the same $n$ and $l$ become non-degenerate in energy in some cases. This can be seen in Table 2, where the quadrupole moment of $q_{\max }^{(\mathrm{sp})}$, the square radius $\left\langle r^{2}\right\rangle$, and the eigenenergies of the single-particle orbitals $\varepsilon_{j}$, for Sc atoms are shown. As one can see, the eigenenergies of the $p_{z}$ states become different from those of $p_{x}$ and $p_{y}$ states. Since the deformation is axially symmetric and thus there is only one specific direction, i.e. the orbitals along $x$ and $y$ axes $\left(p_{x}\right.$ and $p_{y}$ orbitals or $d_{y z}$ and $d_{z x}$ orbitals) remain degenerate. This is similar to the Stark effect 79 if the difference of spherical and deformed effective potentials is treated as an external electric field. We note, however, that the energy difference for the core orbitals is the order of 0.05 Hartree $\simeq 1 \mathrm{eV}$ or less, and is rather small. This is because they have small overlap with the valence wave functions creating the non-spherical effective potential.

On the other hand, for the open-shell outer-most valence electrons, such liftings of degeneracy can be relevant. For example, the outer-most electron of $\mathrm{Al}$ atom occupies the $3 p_{z}$ orbital. If the system is completely spherical, the $3 p_{z}$ orbital is degenerate with the $3 p_{x}$ and $3 p_{y}$ orbitals, while due to the deformation the $3 p_{x}$ and $3 p_{y}$ orbitals are non-degenerate to the $3 p_{z}$ orbital. The eigenenergy of the $3 p_{z}$ orbital is -0.2099 Hartree, while those of the $3 p_{x}$ and $3 p_{y}$ orbitals are 0.0121 Hartree. Due to this degeneracy lifting, the last electron prefers to occupy the $3 p_{z}$ orbital instead of the equal filling of the $3 p_{x}, 3 p_{y}$, and $3 p_{z}$ orbitals, leading to the deformation. It is noted that this degeneracy lifting of $p$ states results from the second-order perturbation: It is caused by the deformation of the core and the $3 s$ orbital, which is induced by the existence of the $3 p_{z}$ electron.

These results suggest that the dominant part of the effective potential is created by the spherical central region. The non-spherical contribution to the effective potential of the valence deformed electrons is sub-dominant and can be treated as small correction.

Screening effect: Let us scrutinize deformations of each orbital with $\operatorname{Sc}(Z=21)$ atom in Table 2 (results for other atoms are also shown in Table C6 C10 in Appendix C). Here, Sc atom has filled $1 s-3 p$ orbitals, in addition to one $3 d_{x y}$ electron and two $4 s$ electrons. The quadrupole moments $q_{z}^{(\mathrm{sp})}$ of the filled $1 s-3 p$ orbitals completely cancel each other, resulting in undeformed spherical core. The square radii of the $1 s-3 p$ orbitals are much smaller than those of $3 d_{x y}$ and $4 s$ orbitals, so that we can essentially regard Sc atom as a system of one $3 d_{x y}$ electron and two $4 s$ electrons orbiting around an almost perfectly inert small spherical core. The electron in the $3 d_{x y}$ in the $\alpha$-spin state is oblate $q_{z}^{(\mathrm{sp})}=-2.1642<0$. This results in an oblate shape of Sc atom as a 
On deformability of atoms

Table 1. Deformation parameters for the selected single-particle orbitals.

\begin{tabular}{llr}
\hline Atom & Orbital & \multicolumn{1}{c}{$\beta_{\max }^{(\mathrm{sp})}$} \\
\hline $\mathrm{Al}$ & $\alpha 3 p_{z}$ & 0.6341 \\
\hline $\mathrm{Sc}$ & $\alpha 3 p_{z}$ & 0.6341 \\
$\mathrm{Sc}$ & $\alpha 3 p_{x}$ & 0.6341 \\
$\mathrm{Sc}$ & $\alpha 3 d_{y z}$ & -0.4530 \\
$\mathrm{Sc}$ & $\alpha 4 s$ & -0.0098 \\
$\mathrm{Sc}$ & $\beta 4 s$ & 0.0830 \\
\hline $\mathrm{Ti}$ & $\alpha 3 p_{x}$ & 0.6341 \\
$\mathrm{Ti}$ & $\alpha 3 p_{z}$ & 0.6341 \\
$\mathrm{Ti}$ & $\alpha 3 d_{z x}$ & -0.4530 \\
$\mathrm{Ti}$ & $\alpha 4 s$ & -0.0063 \\
$\mathrm{Ti}$ & $\beta 4 s$ & -0.0530 \\
\hline $\mathrm{Ni}$ & $\alpha 3 p_{z}$ & 0.6341 \\
$\mathrm{Ni}$ & $\alpha 3 d_{x^{2}-y^{2}}$ & -0.4530 \\
$\mathrm{Ni}$ & $\alpha 3 d_{x y}$ & -0.4530 \\
$\mathrm{Ni}$ & $\alpha 3 d_{y z}$ & -0.4530 \\
$\mathrm{Ni}$ & $\alpha 3 d_{z^{2}}$ & 0.4388 \\
$\mathrm{Ni}$ & $\alpha 4 s$ & -0.0163 \\
\hline $\mathrm{Ga}$ & $\alpha 3 p_{x}$ & 0.6341 \\
$\mathrm{Ga}$ & $\alpha 3 p_{z}$ & 0.6341 \\
$\mathrm{Ga}$ & $\alpha 3 d_{x^{2}-y^{2}}$ & -0.4530 \\
$\mathrm{Ga}$ & $\alpha 3 d_{y z}$ & -0.4530 \\
$\mathrm{Ga}$ & $\alpha 3 d_{z^{2}}$ & 0.4576 \\
$\mathrm{Ga}$ & $\alpha 4 s$ & -0.0008 \\
$\mathrm{Ga}$ & $\alpha 4 p_{z}$ & 0.6341 \\
\hline & &
\end{tabular}

whole, but the value $q_{z}^{(\mathrm{sp})}=-0.4189$ is much smaller than the quadrupole moment of the $3 d_{x y}$ electron. This can be explained by the screening effect: We can see in Table 2 that the electron in the $4 s$ orbital with $\beta$-spin state deforms so significantly that the quadrupole moment of the $\beta 4 s$ electron cancels that of the $\alpha 3 d_{x y}$ orbital. To be more precise, $\left|q_{z}^{(\mathrm{sp})}\right|$ of $\beta 4 s$ is almost the same but slightly smaller than $\left|q_{z}^{(\mathrm{sp})}\right|$ of $\alpha 3 d_{x y}$, which suggests that the quadrupole moment of the $\alpha 3 d_{x y}$ electron is almost but not perfectly cancelled, resulting in a small but finite deformation of Sc atom. On the other hand, the $4 s$ electron in the other spin state (i.e. $\alpha 4 s$ ) is deformed much less than $\beta 4 s$ electron, and does not contribute so much to the screening. This is because the Coulomb repulsion, which is the key for the screening effect (see section 4), is relevant between different spin states, while the Pauli principle makes the effects of the Coulomb repulsion between the same spins weaker.

Similar situations also occur in the other atoms presented in Table 1. For Ti atom (see also Table $\mathrm{C} 8$ in Appendix C), the large deformation is due to the $\alpha 3 d_{y z}$ and the $\alpha 3 d_{z x}$ orbitals, and in total the deformation is prolate $(\beta>0)$. The $\beta 4 s$ orbital is deformed to cancel the deformation of the $\alpha 3 d$ orbitals. The $\alpha 4 s$ orbital is also deformed but because the effects of the Coulomb repulsion is weakened by the Pauli principle, the deformation is much smaller. For Ni atom (Table C9 in Appendix C), the large prolate deformation created by the $\beta 3 d_{z^{2}}$ orbital is partly, but not fully, cancelled by the $\alpha 4 s$ electron $\|$.

\| Since the eigenenergies of $d_{x^{2}-y^{2}}$ and $d_{z^{2}}$ are identical if the system is completely spherical, the 
In the case of Ga atom (see also Table C10 in Appendix C), the large prolate deformation is due to the $\alpha 4 p_{z}$ orbital $q_{z}^{(\mathrm{sp})}=11.1659>0$. With the same argument, we might expect that the $\beta 4 s$ orbital should be largely deformed and cancel that of the $\alpha 4 p_{z}$ orbital. However, the $\beta 4 s$ orbital has rather small quadrupole moment $q_{z}^{(\mathrm{sp})}=0.0139$. It, therefore, does not cancel the deformation of the $\alpha 4 p_{z}$ orbital, resulting in $Q_{z}=11.1576$ of Ga atom as a whole. This is because $\left\langle r^{2}\right\rangle$ of the $4 s$ orbital is much smaller than that of the $\alpha 4 p_{z}$ orbital, and thus the wave function of the $4 s$ orbital does not have enough overlap region with the $\alpha 4 p_{z}$ orbital to cancel its deformation.

We can also see the importance of the radii by comparing Sc, Ti, and Ni data in Tables 2, C8, and C9, respectively. For Sc atom, the quadrupole moment of the valence $d$ electron is almost perfectly cancelled. On the other hand, it is marginally cancelled for $\mathrm{Ti}$ atom, while it is slightly cancelled for $\mathrm{Ni}$ atom. This behavior can be easily understood by noting that radius of the valence $d$ orbital gets smaller as the atomic number increases: $\left\langle r^{2}\right\rangle=3.8,2.9$, and $1.5 \mathrm{Bohr}^{2}$ for $\mathrm{Sc}, \mathrm{Ti}$, and $\mathrm{Ni}$ atoms, respectively. The overlap between the valence $d$ orbital and the $4 s$ orbital gets smaller, which explains why the screening effects gets weaker as the atomic number increases in these atoms.

Moreover, the deformation of the $d$-block atoms becomes further smaller due to the radius of the valence single-particle orbitals, compared to that of the $p$-block atoms. The order of $\sum_{m}\left\langle r^{2}\right\rangle_{n 1}=3\left\langle r^{2}\right\rangle_{n 1}$ for the valence $p$ orbital is the same as that of $Z\left\langle r^{2}\right\rangle$ for the whole atom and thus $|\beta|$ of the $p$-block open-shell atoms are large, whereas the order of $\sum_{m}\left\langle r^{2}\right\rangle_{n 2}=5\left\langle r^{2}\right\rangle_{n 2}$ for the valence $d$ orbital is smaller than that of $Z\left\langle r^{2}\right\rangle$, since the $s$ orbital with the principal quantum number $n+1$ is, in general, also occupied.

Systematic behavior of $\beta$ : With all these physical arguments, we can now fully understand the results in figure 3 . We have found there that the $p$-block and $d$-block atoms can be deformed, but the deformation is much smaller for the $d$-block atoms compared with the $p$-block atoms showing single-particle-like deformation. This result can be explained by noting that the screening effects of the outer-most $s$ orbital are rather significant for the $d$-block atoms, as discussed above for $\mathrm{Sc}, \mathrm{Ti}$, and $\mathrm{Ni}$ atoms. On the other hand, the outer-most valence $p$ orbital of the $p$-block atom has much smaller overlap with the outer-most $s$ orbital, so that the deformation caused by the valence $p$ orbital remains unscreened.

This difference can also be understood by using deformation of the effective potential. As discussed in the beginning of this subsection, the effective potential remains almost spherical, while in the tail region it becomes anisotropic mainly due to the outer-most $p$ or $d$ orbitals. Consequently, such deformation can affect the orbitals which have larger spatial distribution. On the one hand, the outer-most $s$ orbital of the $d$-block atoms, which has comparable $\left\langle r^{2}\right\rangle$ to the valence orbital, is thus significantly affected by the anisotropic effective potential created by the valence $d$ electrons. Accordingly, the $s$ orbital can be deformed largely. On the other hand, the outer-most $s$ orbital of the $p$-block atoms has smaller $\left\langle r^{2}\right\rangle$ than the outer-most

state with the last outer-most electron occupying $d_{x^{2}-y^{2}}$ is almost degenerate with the state with $d_{z^{2}}$. The deformation parameters $\beta$ of these two states may have opposite sign, since $\beta^{(\mathrm{sp})}$ of $d_{x^{2}-y^{2}}$ and $d_{z^{2}}$ orbitals have the same absolute value but with the opposite sign (see 23b and 23c]). Therefore, there exist two states close in energy to each other which have opposite sign of $\beta$ with almost the same absolute value. 
$p$ orbital. Thus, the $s$ orbital cannot be affected by such deformation of the effective potential, and, as a result, deformed largely.

Since deformation of the $p$-block atoms originates from single-particle-like deformation, atoms whose configurations of valence electrons are $p^{1}$ and $p^{4}$ always show prolate $(\beta>0)$ deformation and the last electron occupies $p_{z}$ orbital. In contrast, atoms whose configurations of valence electrons are $p^{2}$ and $p^{5}$ always show oblate $(\beta<0)$ deformation and the last two electrons occupy $p_{x}$ and $p_{y}$ orbital. Since deformation of the $d$-block atoms induces the screening as discussed above, the deformation is small and there is no obvious tendency.

Table 2. Mean-square radius $\left\langle r^{2}\right\rangle_{n l}$, single-particle quadrupole moment $q_{z}^{(\mathrm{sp})}$, and deformation parameter $\beta_{z}^{(\mathrm{sp})}$ for single-particle orbitals of Sc atom calculated by the unrestricted Hartree-Fock method with $6-31+\mathrm{G}$ basis. The single-particle energy of each orbital $\varepsilon_{j}$ and corresponding orbital are also shown. The Hartree atomic unit is used for $\varepsilon_{j},\left\langle r^{2}\right\rangle_{n l}$, and $q_{z}^{(\mathrm{sp})}$.

\begin{tabular}{rlrrrr}
\hline Spin & Orbital & \multicolumn{1}{c}{$\varepsilon_{j}$} & $\left\langle r^{2}\right\rangle_{n l}$ & \multicolumn{1}{c}{$q_{z}^{(\mathrm{sp})}$} & \multicolumn{1}{c}{$\beta_{z}^{(\mathrm{sp})}$} \\
\hline$\alpha$ & $1 s$ & -165.8952 & 0.0073 & 0.0000 & 0.0000 \\
$\alpha$ & $2 s$ & -19.0956 & 0.1393 & 0.0000 & 0.0000 \\
$\alpha$ & $2 p_{z}$ & -15.6861 & 0.1162 & 0.0930 & 0.6341 \\
$\alpha$ & $2 p_{x}$ & -15.6830 & 0.1164 & -0.0466 & -0.3171 \\
$\alpha$ & $2 p_{y}$ & -15.6830 & 0.1164 & -0.0466 & -0.3171 \\
$\alpha$ & $3 s$ & -2.5988 & 1.3396 & -0.0031 & -0.0018 \\
$\alpha$ & $3 p_{x}$ & -1.6232 & 1.6265 & -0.6506 & -0.3171 \\
$\alpha$ & $3 p_{y}$ & -1.6232 & 1.6265 & -0.6506 & -0.3171 \\
$\alpha$ & $3 p_{z}$ & -1.5893 & 1.6107 & 1.2886 & 0.6341 \\
$\alpha$ & $3 d_{x y}$ & -0.3375 & 3.7873 & -2.1642 & -0.4530 \\
$\alpha$ & $4 s$ & -0.2171 & 17.4198 & -0.2152 & -0.0098 \\
\hline$\beta$ & $1 s$ & -165.8951 & 0.0073 & 0.0000 & 0.0000 \\
$\beta$ & $2 s$ & -19.0832 & 0.1391 & 0.0001 & 0.0007 \\
$\beta$ & $2 p_{z}$ & -15.6837 & 0.1162 & 0.0929 & 0.6341 \\
$\beta$ & $2 p_{x}$ & -15.6657 & 0.1161 & -0.0464 & -0.3171 \\
$\beta$ & $2 p_{y}$ & -15.6657 & 0.1161 & -0.0464 & -0.3171 \\
$\beta$ & $3 s$ & -2.5451 & 1.3370 & 0.0387 & 0.0230 \\
$\beta$ & $3 p_{z}$ & -1.5798 & 1.6156 & 1.2925 & 0.6341 \\
$\beta$ & $3 p_{x}$ & -1.5273 & 1.6196 & -0.6478 & -0.3171 \\
$\beta$ & $3 p_{y}$ & -1.5273 & 1.6196 & -0.6478 & -0.3171 \\
$\beta$ & $4 s$ & -0.2051 & 18.5388 & 1.9406 & 0.0830 \\
\hline Total & & & 53.1312 & -0.4189 & \\
\hline
\end{tabular}

Single-particle versus many-body effects: As shown above, the deformations of the electron density in atoms at most originate from the single-particle orbitals of a few valence electrons, and thus there is no collective deformation. Rather, many-body effects in atoms disfavor deformations. With such tiny deformations, the effective potential is slightly deformed only in the surface and the tail (i.e. the valence electron) regions, but the dominant part remains spherical. This justifies the use of spherically symmetric effective potentials or density functionals in the conventional atomic structure calculations [80, 33, 28.

This is in stark contrast with nuclear systems. It is well known that nuclei can be deformed significantly via collective many-body effects, and they often have $|\beta| \gtrsim 0.3[5,81$. In Appendix A we exemplify this with model calculations for nuclear 
many-body systems. In the following section, we attempt to explain with a simple qualitative model why there is no collective deformation in the atoms whereas it exists in atomic nuclei.

\section{Qualitative discussion}

In the previous section and in Appendix A, we have shown that electrons in atoms are much less likely to deform than nucleons in atomic nuclei. We argue in this section that this difference physically originates from the nature of the inter-particle interactions: the repulsive Coulomb interaction between the electrons and the attractive nuclear interaction between the nucleons. It should be noticed that the isoscalar protonneutron interaction, which is strong attractive, has been argued to play an important role in nuclear deformation [6, 7, 8, 9]. While this is true in general, we show in Appendix A that neutron drops can also be deformed even though there is only one type of particles, and thus, there is no neutron-proton interaction. The deformations in the neutron drops can be qualitatively understood by our analytical argument given in this section.

To illustrate this point, we closely follow 82,83 . We consider a system with $N$ particles where the total-spin component, denoted as $S_{z}$, is conserved; $\left[\hat{H}, S_{z}\right]=0$. By taking the $N$-body wave function as a simultaneous eigenstate of $S_{z}$ and $\hat{H}$, the number of particles in each spin state is conserved, and we can regard the system as composed of $N_{\uparrow}$ spin-up and $N_{\downarrow}$ spin-down particles $\left(N=N_{\uparrow}+N_{\downarrow}\right)$. We consider deforming wave functions $\Psi_{\mathrm{sph}}\left(\boldsymbol{r}_{1}, \ldots, \boldsymbol{r}_{N}\right)$ associated with a spherical density with the following coordinate transformation:

$$
\begin{aligned}
& \Psi_{\mathrm{def}}\left(x_{1}, y_{1}, z_{1}, \ldots, x_{N}, y_{N}, z_{N}\right) \\
= & e^{N_{\uparrow}\left(2 \beta_{\uparrow}-\alpha_{\uparrow}\right) / 2} e^{N_{\downarrow}\left(2 \beta_{\downarrow}-\alpha_{\downarrow}\right) / 2} \Psi_{\mathrm{sph}}\left(X_{1}, Y_{1}, Z_{1}, \ldots, X_{N}, Y_{N}, Z_{N}\right),
\end{aligned}
$$

where $X_{j}=e^{\beta_{\uparrow}} x_{j}, Y_{j}=e^{\beta_{\uparrow}} y_{j}$, and $Z_{j}=e^{-\alpha_{\uparrow}} z_{j}$ for $j=1,2, \ldots, N_{\uparrow}$ and $X_{j}=e^{\beta_{\downarrow}} x_{j}$, $Y_{j}=e^{\beta_{\downarrow}} y_{j}$, and $Z_{j}=e^{-\alpha_{\downarrow}} z_{j}$ for $j=N_{\uparrow}+1, N_{\uparrow}+2, \ldots, N$. The coefficient $e^{N_{a}\left(2 \beta_{a}-\alpha_{a}\right) / 2}(a=\uparrow, \downarrow)$ is due to the normalization, which appears since, in the $\alpha_{a} \neq 2 \beta_{a}$ case, the volume of the system is not conserved. Correspondingly, the single-particle densities of up- and down-spin states are also deformed as follows:

$$
\begin{aligned}
& \rho_{\text {def }, \uparrow}(x, y, z)=e^{2 \beta_{\uparrow}-\alpha_{\uparrow}} \rho_{\mathrm{sph}, \uparrow}\left(e^{\beta_{\uparrow}} x, e^{\beta_{\uparrow}} y, e^{-\alpha_{\uparrow}} z\right), \\
& \rho_{\text {def }, \downarrow}(x, y, z)=e^{2 \beta_{\downarrow}-\alpha_{\downarrow}} \rho_{\mathrm{sph}, \downarrow}\left(e^{\beta_{\downarrow}} x, e^{\beta_{\downarrow}} y, e^{-\alpha_{\downarrow}} z\right) .
\end{aligned}
$$

The coefficient $e^{2 \beta_{a}-\alpha_{a}}$ also originates from the normalization coefficient in (27), or equivalently, the particle-number conservation;

$$
\int \rho_{\mathrm{def}, a}(\boldsymbol{r}) d \boldsymbol{r}=\int \rho_{\mathrm{sph}, a}(\boldsymbol{r}) d \boldsymbol{r}=N_{a} .
$$

Due to the saturation property, the atomic nuclei are expected to be deformed with the constant volume. For such deformation, $\alpha_{a}=2 \beta_{a}$ holds. Furthermore, as seen in section 3, atoms are also deformed with $\alpha=2 \beta$. Hence, in the following discussion, we will show the calculation with $\alpha_{a}=2 \beta_{a}$, and results with $\alpha_{a} \neq 2 \beta_{a}$ will be shown in Appendix B, where we will reach essentially the same conclusion as those shown in this section. Note that $\alpha_{a}=2 \beta_{a}$ corresponds to the quadrupole deformation: 
It makes the wave function shrunken in the $x$ - and $y$-axes, and elongated in the $z$-axis directions when $\beta_{a}$ is positive. The quadrupole deformation of a wave function $\left|\Psi_{\mathrm{sph}}\right\rangle$ associated with a spherical density for the Hamiltonian $\hat{H}=\hat{T}+V_{\text {ext }}+V_{\text {int }}$, consisting of the kinetic energy operator $\hat{T}$, central external potential $V_{\text {ext }}$, and the interaction $V_{\text {int }}$, can be equivalently performed with the following canonical transformation:

$$
\left|\Psi_{\text {def }}\right\rangle=e^{\beta_{\uparrow}\left[\hat{H}, \hat{Q}_{\uparrow}\right]} e^{\beta_{\downarrow}\left[\hat{H}, \hat{Q}_{\downarrow}\right]}\left|\Psi_{\text {sph }}\right\rangle .
$$

Here, $\hat{Q}_{a}$ is the quadrupole operator for each spin state defined as

$$
\begin{aligned}
& \hat{Q}_{\uparrow}=m \sum_{j=1}^{N_{\uparrow}}\left[z_{j}^{2}-\frac{1}{2}\left(x_{j}^{2}+y_{j}^{2}\right)\right], \\
& \hat{Q}_{\downarrow}=m \sum_{j=N_{\uparrow}+1}^{N}\left[z_{j}^{2}-\frac{1}{2}\left(x_{j}^{2}+y_{j}^{2}\right)\right],
\end{aligned}
$$

where $m$ and $x_{j}, y_{j}, z_{j}$ are the mass and coordinates of the particles.

When the absolute value of the deformation parameter $\left|\beta_{a}\right|$ is small, the one-body density $\rho_{\mathrm{def}, a}$ of the system is written as follows:

$$
\begin{aligned}
\rho_{\mathrm{def}, a}(x, y, z)= & \rho_{\mathrm{sph}, a}\left(e^{\beta_{a}} x, e^{\beta_{a}} y, e^{-2 \beta_{a}} z\right) \\
= & \rho_{\mathrm{sph}, a}(r)+f_{a}^{(1)}(\boldsymbol{r}) \frac{d \rho_{\mathrm{sph}, a}(r)}{d r}+f_{a}^{(2)}(\boldsymbol{r}) \frac{d^{2} \rho_{\mathrm{sph}, a}(r)}{d r^{2}}+O\left(\beta^{3}\right) \\
f_{a}^{(1)}(\boldsymbol{r})= & \beta_{a} \frac{x^{2}+y^{2}-2 z^{2}}{r}+\beta_{a}^{2} \frac{x^{2}+y^{2}+4 z^{2}}{r} \\
& -\frac{\beta_{a}^{2}}{2} \frac{x^{4}+y^{4}+4 z^{4}}{r^{3}}+\beta_{a}^{2} \frac{2\left(x^{2}+y^{2}\right) z^{2}-x^{2} y^{2}}{r^{3}} \\
= & -\sqrt{\frac{16 \pi}{5}} \beta_{a} r Y_{20}(\theta, \phi)+\beta_{a}^{2}\left[2 r+\sqrt{\frac{16 \pi}{5}} r Y_{20}(\theta, \phi)\right]-\frac{8 \pi}{5} \beta_{a}^{2} r\left[Y_{20}(\theta, \phi)\right]^{2} \\
f_{a}^{(2)}(\boldsymbol{r})= & \frac{\beta_{a}^{2}}{2} \frac{x^{4}+y^{4}+4 z^{4}}{r^{2}}-\beta_{a}^{2} \frac{2\left(x^{2}+y^{2}\right) z^{2}-x^{2} y^{2}}{r^{2}} \\
= & \frac{8 \pi}{5} \beta_{a}^{2} r^{2}\left[Y_{20}(\theta, \phi)\right]^{2}
\end{aligned}
$$

Let us consider how the energy of the system changes when we induce an infinitesimal quadrupole deformation $\left|\beta_{a}\right| \ll 1$ with this transformation to a spherically symmetric system $\rho_{\mathrm{sph}}(\boldsymbol{r})=\rho_{\mathrm{sph}}(r)$.

Kinetic energy: The kinetic energy reads

$$
\begin{aligned}
T_{\text {def }, a} & =\left\langle\Psi_{\text {def }, a}|\hat{T}| \Psi_{\text {def }, a}\right\rangle \\
& =\left(\frac{2}{3} e^{2 \beta_{a}}+\frac{1}{3} e^{-4 \beta_{a}}\right)\left\langle\Psi_{\mathrm{sph}, a}|\hat{T}| \Psi_{\mathrm{sph}, a}\right\rangle \\
& =\left(\frac{2}{3} e^{2 \beta_{a}}+\frac{1}{3} e^{-4 \beta_{a}}\right) T_{\mathrm{sph}, a}
\end{aligned}
$$


One can therefore see that the kinetic energy increases with the deformation

$$
\Delta T_{a}:=T_{\text {def }, a}-T_{\mathrm{sph}, a} \simeq 4 \beta_{a}^{2} T_{\mathrm{sph}, a}>0 .
$$

We also note that the deformation of a spin component does not affect the kinetic energy of the other spin component.

Central external potential energy: The central external potential energy of a system with density $\rho$ is, in general, written as

$$
E_{\mathrm{ext}}=\int \rho(\boldsymbol{r}) V_{\mathrm{ext}}(r) d \boldsymbol{r} .
$$

Therefore, by using (33), the central external potential changes as

$$
\begin{aligned}
& \Delta E_{\mathrm{ext}, a} \\
:= & E_{\mathrm{ext}, a}^{\mathrm{def}}-E_{\mathrm{ext}, a}^{\mathrm{sph}} \\
= & \int\left[\rho_{\mathrm{def}, a}(\boldsymbol{r})-\rho_{\mathrm{sph}, a}(r)\right] V_{\mathrm{ext}}(r) d \boldsymbol{r} \\
\simeq & \int f_{a}^{(1)}(\boldsymbol{r}) \frac{d \rho_{\mathrm{sph}, a}(r)}{d r} V_{\mathrm{ext}}(r) d \boldsymbol{r}+\int f_{a}^{(2)}(\boldsymbol{r}) \frac{d^{2} \rho_{\mathrm{sph}, a}(r)}{d r^{2}} V_{\mathrm{ext}}(r) d \boldsymbol{r} \\
= & \frac{32 \pi}{5} \beta_{a}^{2} \int_{0}^{\infty} \frac{d \rho_{\mathrm{sph}, a}(r)}{d r} V_{\mathrm{ext}}(r) r^{3} d r+\frac{8 \pi}{5} \beta_{a}^{2} \int_{0}^{\infty} \frac{d^{2} \rho_{\mathrm{sph}, a}(r)}{d r^{2}} V_{\mathrm{ext}}(r) r^{4} d r \\
= & -\frac{32 \pi}{5} \beta_{a}^{2} \int_{0}^{\infty} \rho_{\mathrm{sph}, a}(r) \frac{d}{d r}\left[V_{\mathrm{ext}}(r) r^{3}\right] d r+\frac{8 \pi}{5} \beta_{a}^{2} \int_{0}^{\infty} \rho_{\mathrm{sph}, a}(r) \frac{d^{2}}{d r^{2}}\left[V_{\mathrm{ext}}(r) r^{4}\right] d r,
\end{aligned}
$$

where we have assumed $V_{\text {ext }}(r) \sim r^{d}(d>-3)$ at $r \rightarrow 0$ in performing the partial integral in the final line.

Let us consider in particular the power-law type of external potential $V_{\text {ext }}(r)=$ $c r^{d}$ with given constants $c$ and $d$. Substituting this form into $\sqrt{39}$, one obtains

$$
\Delta E_{\mathrm{ext}, a} \simeq\left[-\frac{8}{5}(d+3)+\frac{2}{5}(d+4)(d+3)\right] \beta_{a}^{2} E_{\mathrm{ext}, a}^{\mathrm{sph}}
$$

In the case of the Coulomb potential $(d=-1), 40$ reads

$$
\Delta E_{\mathrm{ext}, a} \simeq-\frac{4}{5} \beta_{a}^{2} E_{\mathrm{ext}, a}^{\mathrm{sph}}>0 .
$$

In the case of the harmonic oscillator potential $(d=2)$, 40 reads

$$
\Delta E_{\mathrm{ext}, a} \simeq 4 \beta_{a}^{2} E_{\mathrm{ext}, a}^{\mathrm{sph}}>0 .
$$

One can therefore see that the external potential energy increases with the deformation for both the Coulomb and harmonic oscillator potentials. As will be discussed later, they represent the Coulomb potential created by the atomic nucleus in atomic systems, and an external harmonic trap for a neutron drop discussed in Appendix A. respectively. We also note that the deformation of a spin component does not affect the potential energy of the other spin component.

Interaction energy: The interaction part of the energy, on the other hand, can either decrease or increase, and hence, favor or disfavor deformation depending on the 
nature of the interaction. Also, as we shall show, the spins play an important role. To see this point, let us consider the direct (Hartree) part of the two-body interaction energy between the $a$ and $b$ spin states characterized by one-body density $\rho_{\text {def, } a}(\boldsymbol{r})$ :

$$
E_{\mathrm{int}, a b}^{\mathrm{def}}=\frac{1}{2} \iint V_{\mathrm{int}}^{a b}\left(\left|\boldsymbol{r}-\boldsymbol{r}^{\prime}\right|\right) \rho_{\mathrm{def}, a}(\boldsymbol{r}) \rho_{\mathrm{def}, b}\left(\boldsymbol{r}^{\prime}\right) d \boldsymbol{r} d \boldsymbol{r}^{\prime},
$$

where $V_{\text {int }}^{a b}$ is the interaction between a particle with spin $a$ and that with spin $b$. The total interaction energy, as long as we only consider the direct part, can then be written as $E_{\text {int, tot }}^{\text {def }}=E_{\text {int, } \uparrow \uparrow}^{\text {def }}+2 E_{\text {int, } \uparrow \downarrow}^{\text {def }}+E_{\text {int, }, \downarrow}^{\text {def }}$. Notice that the multipole expansion of the interaction reads

$$
V_{\mathrm{int}}^{a b}\left(\left|\boldsymbol{r}-\boldsymbol{r}^{\prime}\right|\right)=\sum_{l, m} V_{l}^{a b}\left(r, r^{\prime}\right) Y_{l m}(\theta, \phi) Y_{l m}^{*}\left(\theta^{\prime}, \phi^{\prime}\right) .
$$

Substituting (33) and (44) into (43), one obtains the change of the interaction energy induced by the deformation $\Delta E_{\mathrm{int}, a b}:=E_{\mathrm{int}, a b}^{\mathrm{def}}-E_{\mathrm{int}, a b}^{\mathrm{sph}}$ as

$$
\Delta E_{\mathrm{int}, a b}=\beta_{a}^{2} \Delta \varepsilon_{\mathrm{int}, a b}^{(1)}+\beta_{b}^{2} \Delta \varepsilon_{\mathrm{int}, a b}^{(2)}+\beta_{a} \beta_{b} \Delta \varepsilon_{\mathrm{int}, a b}^{(3)}+O\left(\beta^{3}\right),
$$

with

$$
\begin{aligned}
\Delta \varepsilon_{\mathrm{int}, a b}^{(1)}= & \frac{16 \pi}{5} \int_{0}^{\infty} \int_{0}^{\infty} \frac{d \rho_{\mathrm{sph}, a}(r)}{d r} \rho_{\mathrm{sph}, b}\left(r^{\prime}\right) V_{0}^{a b}\left(r, r^{\prime}\right) r^{3} r^{\prime 2} d r d r^{\prime} \\
& +\frac{4 \pi}{5} \int_{0}^{\infty} \int_{0}^{\infty} \frac{d^{2} \rho_{\mathrm{sph}, a}(r)}{d r^{2}} \rho_{\mathrm{sph}, b}\left(r^{\prime}\right) V_{0}^{a b}\left(r, r^{\prime}\right) r^{4} r^{\prime 2} d r d r^{\prime} \\
\Delta \varepsilon_{\mathrm{int}, a b}^{(2)}= & \frac{16 \pi}{5} \int_{0}^{\infty} \int_{0}^{\infty} \rho_{\mathrm{sph}, a}(r) \frac{d \rho_{\mathrm{sph}, b}\left(r^{\prime}\right)}{d r^{\prime}} V_{0}^{a b}\left(r, r^{\prime}\right) r^{2} r^{\prime 3} d r d r^{\prime} \\
& +\frac{4 \pi}{5} \int_{0}^{\infty} \int_{0}^{\infty} \rho_{\mathrm{sph}, a}(r) \frac{d^{2} \rho_{\mathrm{sph}, b}\left(r^{\prime}\right)}{d r^{\prime 2}} V_{0}^{a b}\left(r, r^{\prime}\right) r^{2} r^{\prime 4} d r d r^{\prime}=\Delta \varepsilon_{\mathrm{int}, b a}^{(1)}, \\
\Delta \varepsilon_{\mathrm{int}, a b}^{(3)}= & \frac{8 \pi}{5} \int_{0}^{\infty} \int_{0}^{\infty} \frac{d \rho_{\mathrm{sph}, a}(r)}{d r} \frac{d \rho_{\mathrm{sph}, b}\left(r^{\prime}\right)}{d r^{\prime}} V_{2}^{a b}\left(r, r^{\prime}\right) r^{3} r^{\prime 3} d r d r^{\prime} .
\end{aligned}
$$

To be more explicit, for the parallel spin states, one finds

$$
\Delta E_{\mathrm{int}, a a}=\beta_{a}^{2}\left(\Delta \varepsilon_{\mathrm{int}, a a}^{(1)}+\Delta \varepsilon_{\mathrm{int}, a a}^{(2)}+\Delta \varepsilon_{\mathrm{int}, a a}^{(3)}\right)+O\left(\beta^{3}\right)
$$

and for the anti-parallel spin states $(a \neq b)$

$$
\Delta E_{\mathrm{int}, a b}=\beta_{\uparrow}^{2} \Delta \varepsilon_{\mathrm{int}, a b}^{(1)}+\beta_{\downarrow}^{2} \Delta \varepsilon_{\mathrm{int}, a b}^{(2)}+\beta_{\uparrow} \beta_{\downarrow} \Delta \varepsilon_{\mathrm{int}, a b}^{(3)}+O\left(\beta^{3}\right) .
$$

First, let us consider the case of atomic nuclei. We note that the interaction between nucleons in vacuum are not yet rigorously known, but it basically comprises of relatively long-range attractive interactions originating from exchanges of several mesons and a short-range repulsive interaction [84, 85, 86, 87, 88. These combined together have a net attractive effect between nucleons, which results in a bound state of a proton and a neutron (i.e. deuteron) [89, 90, and in a large negative scattering length between nucleons [86, 87. We also note that an effective in-medium nucleon-nucleon interaction used in nuclear many-body calculations in general has a net attractive 
effect [91]. For our qualitative discussion in this section, a simple delta-function interaction $V_{\text {int }}^{a b}(\boldsymbol{r})=-g_{a b} \delta(\boldsymbol{r})$ suffices to model this net nuclear interaction between the nucleons. The parameter $g_{a b}$ is positive when the interaction is attractive, such as for nuclear systems, whereas it is negative when the interaction is repulsive. The multipole expansion of this interaction reads

$$
V_{0}^{a b}\left(r, r^{\prime}\right)=V_{2}^{a b}\left(r, r^{\prime}\right)=-g_{a b} \frac{\delta\left(r-r^{\prime}\right)}{r r^{\prime}} .
$$

Substituting (49) into 45] and (46), one obtains

$$
\begin{aligned}
& \Delta E_{\mathrm{int}, a b} \\
\simeq & -\frac{16 \pi g_{a b}}{5} \beta_{a}^{2} \int_{0}^{\infty} \frac{d \rho_{\mathrm{sph}, a}(r)}{d r} \rho_{\mathrm{sph}, b}(r) r^{3} d r-\frac{4 \pi g_{a b}}{5} \beta_{a}^{2} \int_{0}^{\infty} \frac{d^{2} \rho_{\mathrm{sph}, a}(r)}{d r^{2}} \rho_{\mathrm{sph}, b}(r) r^{4} d r \\
& -\frac{16 \pi g_{a b}}{5} \beta_{b}^{2} \int_{0}^{\infty} \rho_{\mathrm{sph}, a}(r) \frac{d \rho_{\mathrm{sph}, b}(r)}{d r} r^{3} d r-\frac{4 \pi g_{a b}}{5} \beta_{b}^{2} \int_{0}^{\infty} \rho_{\mathrm{sph}, a}(r) \frac{d^{2} \rho_{\mathrm{sph}, b}(r)}{d r^{2}} r^{4} d r \\
& -\frac{8 \pi g_{a b}}{5} \beta_{a} \beta_{b} \int_{0}^{\infty} \frac{d \rho_{\mathrm{sph}, a}(r)}{d r} \frac{d \rho_{\mathrm{sph}, b}(r)}{d r} r^{4} d r \\
= & \frac{4 \pi g_{a b}}{5}\left(\beta_{a}-\beta_{b}\right)^{2} \int_{0}^{\infty} \frac{d \rho_{\mathrm{sph}, a}(r)}{d r} \frac{d \rho_{\mathrm{sph}, b}(r)}{d r} r^{4} d r .
\end{aligned}
$$

Thus, on the one hand, the interaction energy for the same spin component does not change with the deformation: $\Delta E_{\text {int }, \uparrow \uparrow}=\Delta E_{\text {int, } \downarrow \downarrow}=0$. This can be also shown using (43) without using the multipole expansion. This is due to a scaling symmetry of the delta function interaction. On the other hand, the interaction energy of the anti-parallel spin states $E_{\text {int, }}^{\text {def }}$ can be non-zero. Indeed, in the nuclear system, the one-body density is more or less constant in the interior region due to its saturation property, $d \rho_{\mathrm{sph}} / d r \simeq 0$, and it suddenly decreases at the surface region $d \rho_{\mathrm{sph}} / d r<0$. Thus, it is reasonable to assume $d \rho_{\mathrm{sph}} / d r \leq 0$ in the most region. Therefore, for the anti-parallel spin states $a \neq b$, the integral of 50 is positive. For the attractive interaction $g_{\uparrow \downarrow}>0$, it is energetically favorable to have $\beta_{a}=\beta_{b}$, so that there is no energy increase with the deformation, i.e. $\Delta E_{\text {int }, \uparrow \downarrow} \simeq 0$. Hence, the spin-up and -down particles deform in the same manner, i.e. $\rho_{\text {def }, \uparrow}(\boldsymbol{r})=\rho_{\text {def }, \downarrow}(\boldsymbol{r})$. We note that we have assumed in the above argument the simplest delta-function interaction. In reality, the modern nuclear effective interaction, such as the Skyrme interaction, contains the terms simulating finite-range effects (e.g., $t_{1}$ and $t_{2}$ terms in A.2). This finite-range effects can make the energy of the system decrease with the nuclear deformation, in contrast to what have been found above with a delta interaction. The above argument can thus only explain why the condition $\beta_{a}=\beta_{b}$ is favored: Once the nuclear deformation occurs with the finite-range effects or other reasons, the nuclei deform in a spin-independent manner due to the attractive nature of interaction.

In contrast, if the interaction was repulsive $g_{\uparrow \downarrow}<0$, the energy can decrease with the deformation $\Delta E_{\text {int }, \uparrow \downarrow}<0$. In particular, the condition $\beta_{a}=-\beta_{b}$ is favored, so that the spin-up and spin-down particles undergo the opposite quadrupole deformation. They cancel with each other when one considers the total density $\rho_{\text {def }}(\boldsymbol{r})=\rho_{\text {def }, \uparrow}(\boldsymbol{r})+\rho_{\text {def, } \downarrow}(\boldsymbol{r})$, so that the net deformation of the whole system is suppressed.

Next, let us consider the case of atoms. The multipole expansion of the Coulomb 
interaction reads

$$
V_{l}\left(r, r^{\prime}\right)=\frac{4 \pi}{2 l+1} \frac{r_{<}^{l}}{r_{>}^{l+1}},
$$

where $r_{<}$and $r_{>}$are smaller and greater ones of $r$ and $r^{\prime}$, respectively. Substituting (51) into 46), one obtains

$$
\begin{aligned}
\Delta \varepsilon_{\mathrm{int}, a b}^{(1)}= & \frac{64 \pi^{2}}{5} \int_{0}^{\infty} \int_{0}^{\infty} \frac{d \rho_{\mathrm{sph}, a}(r)}{d r} \rho_{\mathrm{sph}, b}\left(r^{\prime}\right) \frac{1}{r_{>}} r^{3} r^{\prime 2} d r d r^{\prime} \\
& +\frac{16 \pi^{2}}{5} \int_{0}^{\infty} \int_{0}^{\infty} \frac{d^{2} \rho_{\mathrm{sph}, a}(r)}{d r^{2}} \rho_{\mathrm{sph}, b}\left(r^{\prime}\right) \frac{1}{r_{>}} r^{4} r^{\prime 2} d r d r^{\prime} \\
= & \frac{16 \pi^{2}}{5} \int_{0}^{\infty} \int_{0}^{\infty} \theta\left(r-r^{\prime}\right) \frac{d \rho_{\mathrm{sph}, a}(r)}{d r} \rho_{\mathrm{sph}, b}\left(r^{\prime}\right) r^{2} r^{\prime 2} d r d r^{\prime}, \\
\Delta \varepsilon_{\mathrm{int}, a b}^{(3)}= & \frac{32 \pi^{2}}{25} \int_{0}^{\infty} \int_{0}^{\infty} \frac{d \rho_{\mathrm{sph}, a}(r)}{d r} \frac{d \rho_{\mathrm{sph}, b}\left(r^{\prime}\right)}{d r^{\prime}} \frac{r_{<}^{2}}{r_{>}^{3}} r^{3} r^{\prime 3} d r d r^{\prime} \\
= & \frac{32 \pi^{2}}{25} \int_{0}^{\infty} \int_{0}^{\infty} \frac{d \rho_{\mathrm{sph}, a}(r)}{d r} \frac{d \rho_{\mathrm{sph}, b}\left(r^{\prime}\right)}{d r^{\prime}}\left[\theta\left(r-r^{\prime}\right) r^{\prime 5}+\theta\left(r^{\prime}-r\right) r^{5}\right] d r d r^{\prime},
\end{aligned}
$$

where $\theta(r)$ is the Heaviside step function defined by

$$
\theta\left(r-r^{\prime}\right)= \begin{cases}0 & \left(r<r^{\prime}\right) \\ 1 & \left(r>r^{\prime}\right)\end{cases}
$$

In the atomic system, the one-body density $\rho_{\mathrm{sph}}$ is generally a decreasing function of $r$, except for the surface region (which will be discussed in this section), so that it is reasonable to assume $d \rho_{\mathrm{sph}} / d r \leq 0$ in the most region. Hence, one can find $\Delta \varepsilon_{\mathrm{int}, a b}^{(1)}=\Delta \varepsilon_{\mathrm{int}, b a}^{(2)}<0$ and $\Delta \varepsilon_{\mathrm{int}, a b}^{(3)}>0$. One can also see for the parallel spin states

$$
\begin{aligned}
& \Delta \varepsilon_{\mathrm{int}, a a}^{(1)}+\Delta \varepsilon_{\mathrm{int}, a a}^{(2)}+\Delta \varepsilon_{\mathrm{int}, a a}^{(3)} \\
& =\frac{32 \pi^{2}}{5} \int_{0}^{\infty} \int_{0}^{\infty} \theta\left(r-r^{\prime}\right) \frac{d \rho_{\mathrm{sph}, a}(r)}{d r} \rho_{\mathrm{sph}, a}\left(r^{\prime}\right)\left(r^{2}-r^{\prime 2}\right) r^{\prime 2} d r d r^{\prime}<0 .
\end{aligned}
$$

Therefore, when one considers the interaction energy between the parallel spin [equation (47)], one finds that the right-hand side of is negative, and thus the Coulomb interaction favors deformation. This is a well-known fact in a nuclear fission problem, when the deformed Coulomb energy is evaluated with the liquiddrop model [92, 5]. In addition, if one considers the interaction between the different spin [equation (48]), deformation with the different $\operatorname{sign} \beta_{\uparrow}=-\beta_{\downarrow}$ is energetically favored because of the positive $\Delta \varepsilon_{\text {int, }, \downarrow}^{(3)}$. The effect of $\Delta \varepsilon_{\text {int, }, \downarrow}^{(1)}$ and $\Delta \varepsilon_{\text {int, }, \downarrow}^{(2)}$ also favors deformation because $\Delta \varepsilon_{\text {int, }, \downarrow}^{(1)}=\Delta \varepsilon_{\text {int, } \downarrow \uparrow}^{(2)}<0$. Therefore, the Coulomb repulsive interaction favors spin-dependent deformation: $\rho_{\uparrow}$ and $\rho_{\downarrow}$ makes the opposite quadrupole deformations. When one considers the deformation of the total density $\rho_{\text {def }}(\boldsymbol{r})=\rho_{\text {def, } \uparrow}(\boldsymbol{r})+\rho_{\text {def, } \downarrow}(\boldsymbol{r})$, they cancel with each other and the total deformation would be small. These conclusions are in line with those for the repulsive delta function interaction. However, in contrast to the delta function interaction, there exists nonvanishing contribution from the parallel spin states $\Delta E_{\mathrm{int}, a a}$. 
Overall discussion: From these qualitative discussions, we can see that difference in the nature of the interaction is the key in understanding why atoms do not tend to deform collectively while nuclei do. The kinetic energy and the central external potential energy increase with the deformation, hence disfavor the deformation (see (37), (41), and (42)). The interaction energy, on the other hand, may decrease with the deformation depending on the nature of the interaction. In this connection, we mention that the well-known Hund rule [75, 74, 76, 77, 78, is also intimately related to the repulsive nature of electron-electron interaction, as has been argued in 93. If this decrease is greater than the increase of the kinetic and external potential energies, the system would deform. One finds above that an attractive short-range interaction, modeled by the delta function interaction, favors deformation in a spin-independent manner $\beta_{\uparrow}=\beta_{\downarrow}$ (see (50)). The repulsive Coulomb interaction energy, on the other hand, favors spin-dependent deformation $\beta_{\uparrow}=-\beta_{\downarrow}$, in a manner to cancel with each other the deformation of the total density $\rho_{\text {def }}(\boldsymbol{r})=\rho_{\text {def, } \uparrow}(\boldsymbol{r})+\rho_{\text {def, } \downarrow}(\boldsymbol{r})$ (see (54) and its discussion below). The former represents nuclear systems, and the latter represents atomic systems. The above results thus qualitatively explain why nuclear systems can deform significantly while atomic systems cannot. Moreover, according to the discussion with short-range repulsive interaction $\left(g_{\uparrow \downarrow}<0\right)$, this behavior depends on whether the interaction is repulsive or attractive, while the range of the interaction does not play an important role. The above argument based on two internal states, spin up and down states, can be readily extended to nuclear systems by considering four internal states, proton-up, proton-down, neutron-up, neutron-down states. While this provides a qualitative way to understand the nuclear deformations, it is important to notice that there are several spin-isospin channels in a two-nucleon system, and the relative strength of a nucleon-nucleon interaction for different channels may become rather important in nuclear structure. In particular, it is known that the protonneutron isoscalar interaction plays an important role in nuclear deformation, as has been discussed in $[6,7,8,9$. While this is true in general, we notice that the neutron drops can also be deformed even without the proton-neutron isoscalar interaction, as shown in Appendix A.

This model discussion is closely related to the screening effects of electrons numerically found and discussed in section 3. The screening effect occurs due to the repulsive Coulomb interaction, which aims to decrease any charge imbalance. If, for example, the spin-up electrons make a positive quadrupole deformation, the down spins attempt to make a negative quadrupole deformation to attain the charge neutrality. Therefore, the above argument based on the multipole component of the interaction is consistent with the screening effect. Indeed, we have found in section 3 that the deformation of the valence $d$-orbital is cancelled by the deformation of the opposite spin in the $s$-orbital for the $d$-block atoms. This is in line with the above model calculation. We have also found in section 3 that the deformations of atoms occur only in the valence electron region for $p$ - and $d$-block atoms. We can also explain this by using the above model: The central external potential energy, favoring spherical shape, is great in the central region. Therefore, even if the interaction energy is negative and favors deformation, it is difficult to overcome the positive potential energy. It is thus rather unlikely for the central region of the atoms to undergo significant deformations. In the valence electron region, on the other hand, the potential energy becomes small and it is easier for the interaction energy to overcome it. This qualitatively explains why atoms can be deformed only in the valence electron region. 
As the above discussions for the quadrupole deformation may also apply to the higher order multipole deformations at any order, we can conjecture the following statement for general infinitesimal deformations: The multipole component of the inter-particle interaction must be attractive for the system to undergo significant collective deformations. The Coulomb interaction between the electrons has repulsive multipole components for all orders, and therefore the deformations of up-spin and down-spin states cancel with each other for all multipole orders, resulting in a small total deformation as numerically found in the previous section. The nuclear interaction, on the other hand, may undergo spin-independent deformations when a multipole component of the interaction is attractive and strong enough.

We note that we have assumed in the above model argument that $d \rho_{\mathrm{sph}} / d r \leq 0$. While this assumption is valid for closed shell atoms and also for open-shell atoms in their central region, the electron density usually oscillates for open-shell atoms in the surface region: The electron density in the surface region is mostly determined by the wave function of the outer-most valence electrons $\psi_{n l m s}(r, \theta, \phi)$, which show oscillations with $r$ when $n>1$. Thus, the above model argument in the valence electron region should be taken with some cautions.

We also note that we have neglected in the above discussion the exchangecorrelation part in evaluating the interaction energy [equation (43)]. In the HartreeFock theory and the DFT, the exchange or exchange-correlation part is generally found to be sub-dominant than the direct part contributions for electrons in atoms [80, 54, 33, 94. We have, therefore, only considered the direct part in our basic qualitative discussions above, while one needs to include the exchange-correlation part for more elaborated descriptions. In particular, for electrons in the surface region of atoms (i.e. the valence orbital), the exchange-correlation part becomes more important compared with the central region, because the density is rather small and thus the electrons are strongly correlated [95, 80, 54 . Our model discussion on the surface region of atoms, therefore, should be at best qualitative one. In the nuclear DFT, on the other hand, the direct and exchange parts are usually not considered separately. Rather, a short-range attractive effective interaction $V_{\text {int }}\left(\left|\boldsymbol{r}-\boldsymbol{r}^{\prime}\right|\right)$ in (43) is often used whose shape and parameters are chosen to reproduce well nuclear properties measured in experiments [96, 97, 91. Thus, the above argument based on (43), ostensibly considering only the direct part of interaction energy, naturally contains many-body correlation effects, including those due to the three-body and tensor interaction, and therefore should be valid even though atomic nuclei are rather strongly correlated.

While we have shown that atoms in their ground states are unlikely to be deformed significantly, we may expect larger deformations in more exotic atomic systems. As can be seen in our qualitative discussion above, atoms may be deformed if the condition $d \rho_{\mathrm{sph}} / d r \leq 0$ does not hold or if the exchange-correlation term dominates the direct term. These are often the cases for highly excited atoms. In particular, we can expect that Rydberg atoms [98, 99, 100, which have electrons in excited states with extremely large principal quantum numbers, may show significant deformations. While the Rydberg atoms with one electron excited to such orbital will trivially show a deformed shape representing the single-particle orbital, they may show non-trivial collective deformations when more than one electrons are excited in the Rydberg orbits [100]. 


\section{Conclusion}

We have investigated numerically whether the electron density distribution of isolated atoms can be deformed. To this end, we have calculated the deformation parameters for various atoms with a wide variety of different many-body methods, and have found that the noble-gas, half-shell-closed, and $s$-block atoms are spherical, while the $p$ - and $d$-block atoms are deformed. The deformations of the $p$ - and $d$-block atoms are neither collective nor significantly deformed. We have shown that the core part remains spherical even for these atoms, and their deformations originate from a few valence electrons. Therefore, the deformations of the atoms are at most of a singleparticle nature of a few valance electrons: Atoms do not deform collectively, in contrast to nuclei. We have also shown that the many-body effects of electrons in atoms tend to make the deformations smaller due to the screening effect.

Owing to the deformation, the self-consistent effective mean-field potential for $p$ and $d$-block atoms is slightly deformed in the surface and the tail region. Therefore, the core electrons are still eigenstates of angular momentum and are described by single spherical harmonics, whereas the valence electrons are not. We have found numerically that the degeneracy of the valence electrons are indeed lifted due to this effect, but the effect is 0.05 Hartree or much less. Hence, calculations of the atomic structure with spherical symmetry are still justified.

We have compared the atomic deformation to the nuclear deformation by using a qualitative model. We have argued that the difference between them originates from the properties of the inter-particle interactions. On the one hand, in the case of atomic nuclei, the interaction is of attractive in net, and the deformation of the spin-up component favors to be the same as that of the spin-down component. Consequently, the collective deformation can occur. On the other hand, in the case of atoms, the interaction is purely repulsive, and the deformation of the spin-up component favors to be the opposite of that of the spin-down component, cancelling each other. Accordingly, the collective deformation does not occur. The apparent difference originates from the sign of the inter-particle interaction, rather than its range.

While the collective deformations of nuclei have clearly been observed by the gamma-ray spectroscopy of their rotational spectra of (1), the atomic deformations studied in this work are neither significant nor collective, and therefore should not show similar rotational spectra. One rather needs to directly probe the density distribution of the electrons to experimentally test the deformations found by our quantum chemical calculations. It would be rather challenging but we expect that they may be directly observed with recent atomic and molecular physics technology, such as photo-ionization microscopy [101, 102, or tomographic imaging [103].

\section{Acknowledgment}

We thank Gianluca Colò, Yoshiko Kanada-En'yo, Haozhao Liang, Takashi Nakatsukasa, and Kenichi Yoshida for fruitful discussion. The RIKEN iTHEMS program, the JSPS Grant-in-Aid for JSPS Fellows under Grant No. JP19J20543, and JSPS KAKENHI Grant Nos. JP19K03861, JP19K21028, and JP21H00116 are acknowledged. The numerical calculations were performed on cluster computers at the RIKEN iTHEMS program. 


\section{Appendix A. Deformation of neutron drop in a harmonic trap}

In this Appendix, we discuss an illustrative case of deformed systems: that is, a neutron drop trapped in a spherical harmonic potential. Even though neutron drops are fictitious, they provide useful systems to benchmark many-body theories [104, 105, 106, 107, 108, 109, 110, 111, As the density can be controlled by changing the strength of the trapping potential. They have also been utilized in connection with neutronrich nuclei and neutron stars [112]. By using this model, we show in this Appendix that, within HF theory, the nuclear systems are indeed collectively deformed due to the attractive nuclear force: The systems are collectively deformed when the inter-particle attraction is strong enough, while they do not if the interaction is repulsive.

Although the interaction between neutrons is attractive, in contrast to the interaction between electrons, typical nuclear interactions are not strong enough so that systems consisting solely of neutrons do not bound by themselves. To study properties of many-neutron systems, one thus needs to introduce an external confining potential, analogously to the electron-nucleus external potential in atoms. In this Appendix, we use an isotropic harmonic oscillator potential,

$$
V_{\mathrm{ext}}(r)=\frac{1}{2} m_{n} \Omega^{2} r^{2},
$$

where $m_{n}=939.6 \mathrm{MeV} / c^{2}$ is the neutron mass [113], to localize the neutrons in neutron drops. In this study, we set $\hbar \Omega=5 \mathrm{MeV}$.

To take into account the interaction between neutrons, we employ the HF theory with the Skyrme effective interaction [96, 97. The Skyrme interaction is a zero-range force with momentum and density dependences, given by

$$
\begin{aligned}
v_{\mathrm{Sk}}= & t_{0}\left(1+x_{0} P_{\sigma}\right) \delta\left(\boldsymbol{r}_{1}-\boldsymbol{r}_{2}\right)-\frac{t_{1}}{8}\left(1+x_{1} P_{\sigma}\right)\left[\overleftarrow{\nabla}^{2} \delta\left(\boldsymbol{r}_{1}-\boldsymbol{r}_{2}\right)+\delta\left(\boldsymbol{r}_{1}-\boldsymbol{r}_{2}\right) \vec{\nabla}^{2}\right] \\
& +\frac{t_{2}}{4}\left(1+x_{2} P_{\sigma}\right)\left[\overleftarrow{\nabla} \delta\left(\boldsymbol{r}_{1}-\boldsymbol{r}_{2}\right) \cdot \vec{\nabla}\right]+\frac{t_{3}}{6}\left(1+x_{3} P_{\sigma}\right) \delta\left(\boldsymbol{r}_{1}-\boldsymbol{r}_{2}\right)\left\{\rho\left(\frac{\boldsymbol{r}_{1}+\boldsymbol{r}_{2}}{2}\right)\right\}^{\alpha} \\
& +i \frac{W_{0}}{4}\left[\overleftarrow{\nabla} \times \delta\left(\boldsymbol{r}_{1}-\boldsymbol{r}_{2}\right) \vec{\nabla}\right] \cdot\left(\boldsymbol{\sigma}_{1}+\boldsymbol{\sigma}_{2}\right),
\end{aligned}
$$

where $\vec{\nabla}=\vec{\nabla}_{1}-\vec{\nabla}_{2}$ acting on the right and $\overleftarrow{\nabla}=\overleftarrow{\nabla}_{1}-\overleftarrow{\nabla}_{2}$ acting on the left, and $P_{\sigma}=\left(1+\boldsymbol{\sigma}_{1} \cdot \boldsymbol{\sigma}_{2}\right) / 2$ is the spin-exchange operator. The coefficients $t_{i}, x_{i}(i=0$ $3), \alpha$, and $W_{0}$ are the parameters to be adjusted to properties of the homogeneous nucleon gas, called nuclear matter, and experimentally observed nuclear properties. Among these, the $t_{0}$ and $t_{3}$ terms determine the bulk property of nuclei. The former is attractive, and the latter with density dependence $(\alpha>0)$ is repulsive. The repulsive $t_{3}$ term, which becomes dominant over the other terms at high densities, is responsible for the saturation of nuclear matter density.

In our study, we adopt the $\mathrm{SkM}^{*}$ parameter set 114 given in Table A1. In order to see how the deformation property depends on the strength and the sign of the interaction, we scale the strength parameters of the Skyrme interaction as $t_{i} \rightarrow f t_{i}$ for $i=0,1,2$ and $t_{3} \rightarrow|f| t_{3}$ with $f$ being varied within the range $[-1,1]$, where $f=1$ corresponds to the original. The $t_{3}$ term is kept repulsive to avoid collapse of the system. In our numerical calculations, reflection and axial symmetries are imposed.

To find HF solutions for a neutron drop, we perform several HF iterations for each system starting from prolate and oblate configurations. Most often, a prolate (oblate) 
Table A1. Values of $t_{0}\left(\mathrm{MeV} \mathrm{fm}^{3}\right), t_{1}\left(\mathrm{MeV} \mathrm{fm}^{5}\right), t_{2}\left(\mathrm{MeV} \mathrm{fm}^{5}\right), t_{3}$ $\left(\mathrm{MeV} \mathrm{fm}^{3(1+\alpha)}\right), x_{0}, x_{1}, x_{2}, x_{3}, \alpha$, and $W_{0}\left(\mathrm{MeV} \mathrm{fm}^{5}\right)$ of the $\mathrm{SkM}^{*}$ parameter set [114.

\begin{tabular}{cccccccccc}
\hline$t_{0}$ & $t_{1}$ & $t_{2}$ & $t_{3}$ & $x_{0}$ & $x_{1}$ & $x_{2}$ & $x_{3}$ & $\alpha$ & $W_{0}$ \\
\hline-2645 & 410 & -135 & 15595 & 0.09 & 0 & 0 & 0 & $1 / 6$ & 130 \\
\hline
\end{tabular}

initial condition ends up with a prolate (oblate) solution for open-shell systems. For closed-shell systems, on the other hand, a spherical solution is obtained regardless of the initial deformation if it is energetically favored.

Figure A1 shows the deformation parameters of the neutron drops ${ }^{8-40} n$ with attractive $(f>0)$ interactions. Here, ${ }^{N} n$ denotes the system with $N$ neutrons. The spherical solutions are shown by open circles. The prolate and oblate solutions are respectively plotted by solid and open symbols. Squares, diamonds, and triangles correspond to the solutions for $f=1,0.6$, and 0.2 , respectively. As can be seen from the figure, many of the neutron drops examined here have both prolate and oblate solutions, often at similar energies to each other. The quadrupole deformation tends to be smaller as the strengths of the attractive interaction decrease. This behavior of the deformation can be understood as follows: as the interaction is weakened, the effect of the spherical external field becomes more significant relative to the interaction, which is consistent with the discussion shown in section 4. As a result, the system tends to adjust its density distribution closer to the spherical shape to reduce the potential energy of the external field. The systems with $N=8,16,20,32,34$, and 40 are spherical for all the values of the scaling parameter $f$. They correspond to closed-shell configurations, e.g., $\left(1 s_{1 / 2}\right)^{2}\left(1 p_{3 / 2}\right)^{4}\left(1 p_{1 / 2}\right)^{2}$ for ${ }^{8} n,\left(1 s_{1 / 2}\right)^{2}\left(1 p_{3 / 2}\right)^{4}\left(1 p_{1 / 2}\right)^{2}\left(1 d_{5 / 2}\right)^{6}\left(2 s_{1 / 2}\right)^{2}$ for ${ }^{16} n$, and $\left(1 s_{1 / 2}\right)^{2}\left(1 p_{3 / 2}\right)^{4}\left(1 p_{1 / 2}\right)^{2}\left(1 d_{5 / 2}\right)^{6}\left(2 s_{1 / 2}\right)^{2}\left(1 d_{3 / 2}\right)^{4}$ for ${ }^{20} n$.

We have also carried out HF calculations with repulsive $(f<0)$ interactions, but we obtained spherical solutions for only a few systems with closed-shell configurations, and no deformed solution was found. Although the pairing correlation would stabilize the convergence and allows us to study repulsive systems as well as the attractive ones, in this appendix we only consider systems with variable attractive interaction within the HF theory.

In figures $\mathrm{A} 2$ and $\mathrm{A} 3$, we show the neutron density distributions of prolate and oblate solutions, respectively, of ${ }^{14} n$ for different values of $f$. In both cases, we see clearly that the density becomes tighter and more deformed as $f$ changes from 0.2 to 1.

Evidently, neutrons trapped in an isotropic harmonic-oscillator potential can be collectively deformed for open-shell systems, in contrast to electrons in atoms, as is argued in section 4. As expected, the spherically symmetric external field tends to suppress the deformation parameter as well as the radius of the system. This conclusion is consistent with the qualitative discussion in section 4

\section{Appendix B. Generalization of Section 4}

In this Appendix, we will show the general case (i.e. $\alpha_{a} \neq 2 \beta_{a}$ ) of the calculations shown in section 4 . Firstly, the single-particle densities of up- and down-spin states 


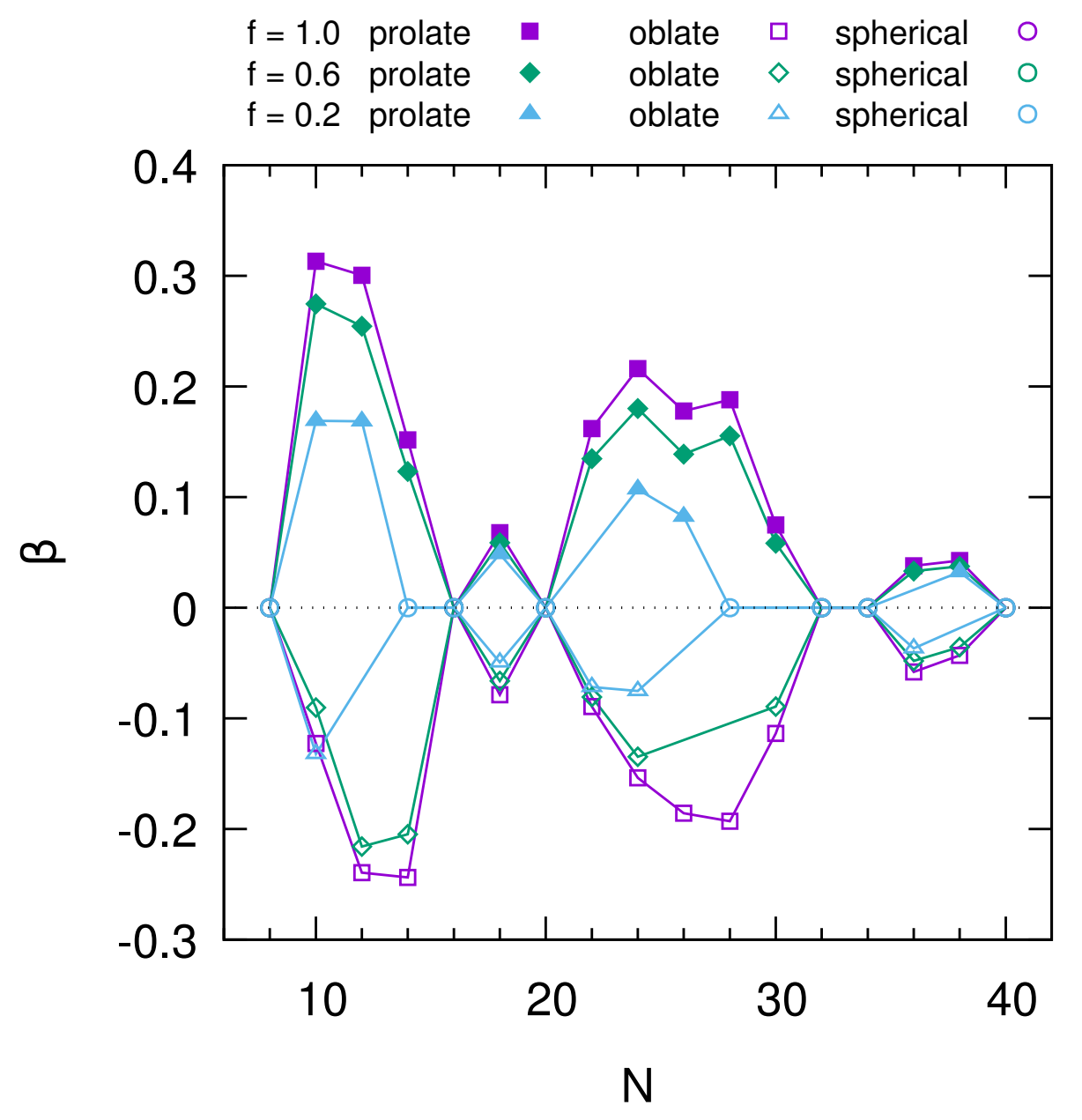

Figure A1. Deformation parameters of HF solutions for the neutron drops ${ }^{8-40} n$ with $\hbar \Omega=5 \mathrm{MeV}$. Here, $f$ is the scaling factor of the strengths of Skyrme interaction (see the main text for details). When there are two solutions both on prolate and oblate sides, both are plotted in the figure.

are defined as follows:

$\rho_{\text {def, } a}(\boldsymbol{r})=\frac{1}{2} \sum_{j=1}^{N} \int \Psi_{\text {def }}^{*}\left(\boldsymbol{r}_{1}, \ldots, \boldsymbol{r}_{N}\right)\left(1 \pm \sigma_{z j}\right) \delta\left(\boldsymbol{r}-\boldsymbol{r}_{j}\right) \Psi_{\text {def }}\left(\boldsymbol{r}_{1}, \ldots, \boldsymbol{r}_{N}\right) d \boldsymbol{r}_{1} \ldots d \boldsymbol{r}_{N}$,

where + in $1 \pm \sigma_{z j}$ for $a=\uparrow$ and - for $a=\downarrow$. For simplicity, the spin coordinates of particles ( $\uparrow$ for particle $j=1,2, \ldots, N_{\uparrow}$ and $\uparrow$ for particle $j=N_{\uparrow}+1$, $\left.N_{\uparrow}+2, \ldots, N\right)$ are omitted. Using the notation of $\boldsymbol{R}_{a}, \boldsymbol{r}, \boldsymbol{R}_{j}$, and $\boldsymbol{r}_{j}$ which denote $\boldsymbol{R}_{a}=\left(e^{\beta_{a}} x, e^{\beta_{a}} y, e^{-\alpha_{a}} z\right), \boldsymbol{r}=(x, y, z), \boldsymbol{R}_{j}=\left(e^{\beta_{j}} x, e^{\beta_{j}} y, e^{-\alpha_{j}} z\right), \boldsymbol{r}_{j}=\left(x_{j}, y_{j}, z_{j}\right)$, 


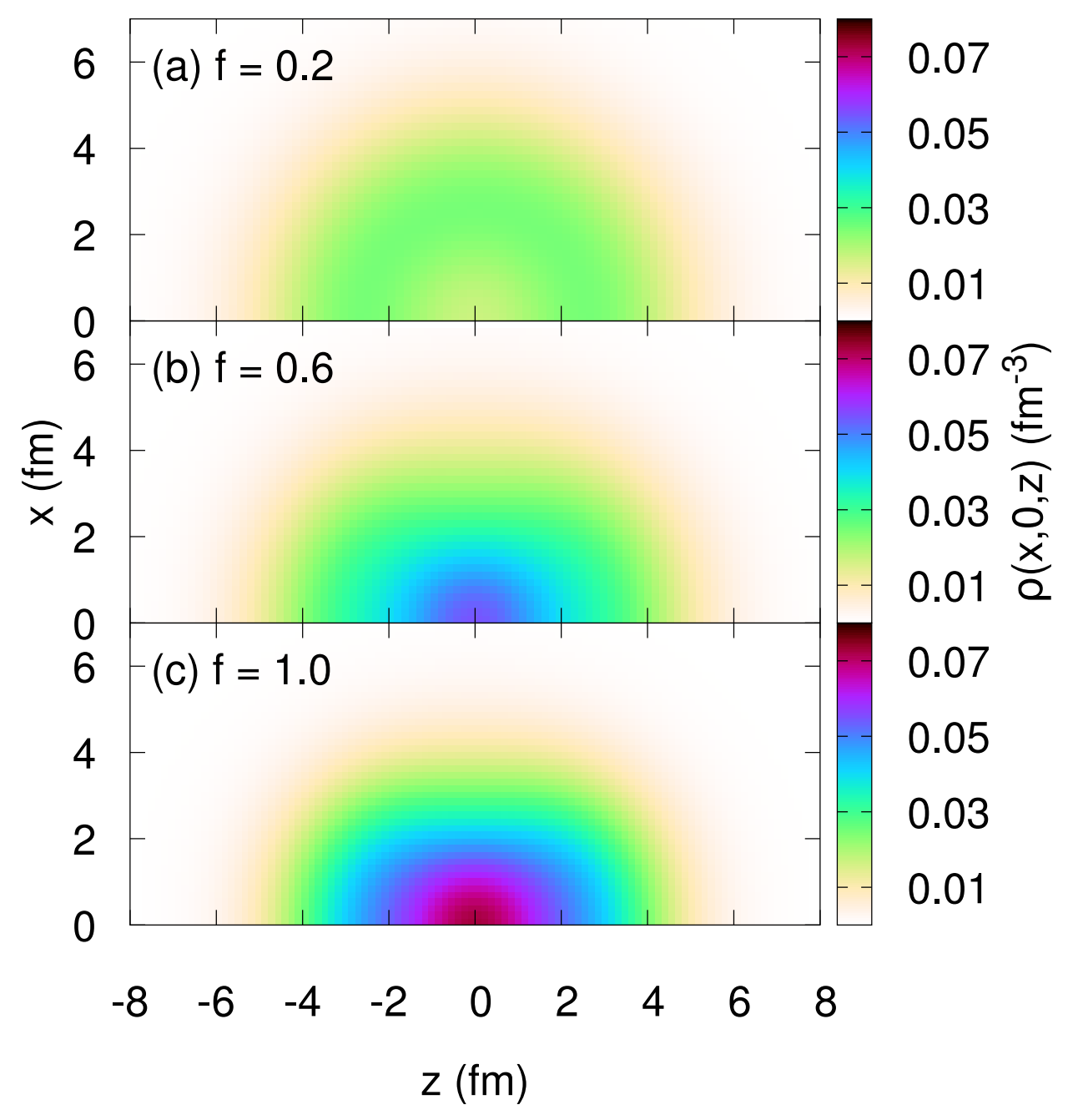

Figure A2. Densities of the prolate HF solutions on $x z$ plane of ${ }^{14} n$ for (a) $f=0.2$, (b) $f=0.6$, and (c) $f=1.0$. The horizontal $(z)$ axis is the axis of symmetry.

and the relation $\delta\left(\boldsymbol{r}-\boldsymbol{r}_{j}\right)=e^{-\left(\alpha_{a}-2 \beta_{a}\right)} \delta\left(\boldsymbol{R}_{a}-\boldsymbol{R}_{j}\right)$, one obtains

$\rho_{\text {def, } a}(\boldsymbol{r})$

$=\frac{1}{2} \sum_{j=1}^{N} \int \Psi_{\mathrm{sph}}^{*}\left(\boldsymbol{R}_{1}, \ldots, \boldsymbol{R}_{N}\right)\left(1 \pm \sigma_{z j}\right) \delta\left(\boldsymbol{r}-\boldsymbol{r}_{j}\right) \Psi_{\mathrm{sph}}\left(\boldsymbol{R}_{1}, \ldots, \boldsymbol{R}_{N}\right) d \boldsymbol{R}_{1} \ldots d \boldsymbol{R}_{N}$

$=\frac{1}{2} \sum_{j=1}^{N} e^{2 \beta_{a}-\alpha_{a}} \int \Psi_{\mathrm{sph}}^{*}\left(\boldsymbol{R}_{1}, \ldots, \boldsymbol{R}_{N}\right)\left(1 \pm \sigma_{z j}\right) \delta\left(\boldsymbol{R}_{a}-\boldsymbol{R}_{j}\right) \Psi_{\mathrm{sph}}\left(\boldsymbol{R}_{1}, \ldots, \boldsymbol{R}_{N}\right) d \boldsymbol{R}_{1} \ldots d \boldsymbol{R}_{N}$

$=e^{2 \beta_{a}-\alpha_{a}} \rho_{\mathrm{sph}, a}\left(\boldsymbol{R}_{a}\right)$. 


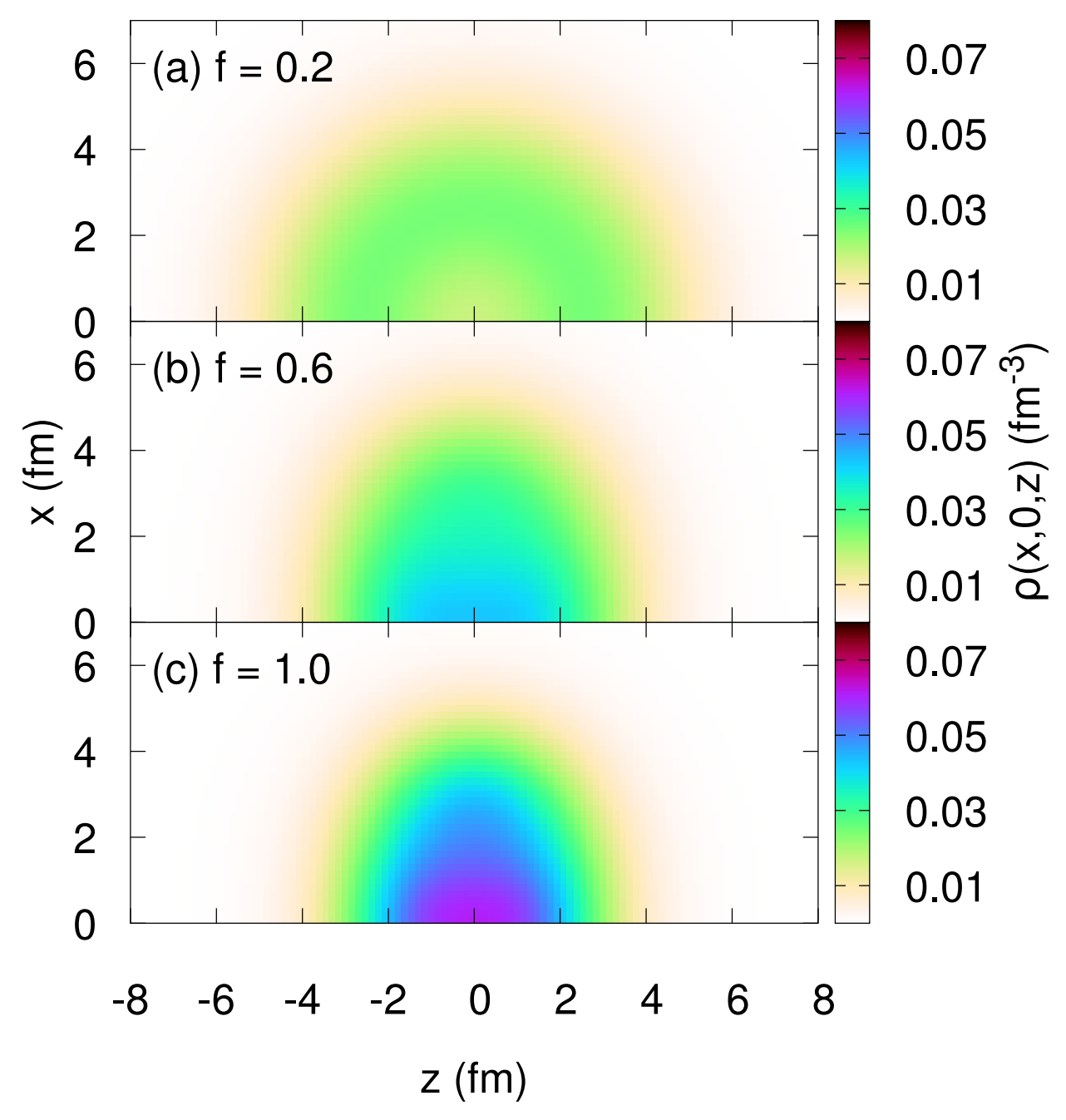

Figure A3. Same as figure A2 but for the oblate HF solutions.

First of all, when the absolute value of the deformation parameters $\left|\alpha_{a}\right|$ and $\left|\beta_{a}\right|$ 
are small, the one-body density $\rho_{\text {def, } a}$ of the system reads

$$
\begin{aligned}
\rho_{\mathrm{def}, a}(x, y, z)= & \rho_{\mathrm{sph}, a}\left(e^{\beta_{a}} x, e^{\beta_{a}} y, e^{-\alpha_{a}} z\right) \\
= & {\left[1+\left(2 \beta_{a}-\alpha_{a}\right)+\frac{1}{2}\left(2 \beta_{a}-\alpha_{a}\right)^{2}\right] \rho_{\mathrm{sph}, a}(r) } \\
& +f_{a}^{(1)}(\boldsymbol{r}) \frac{d \rho_{\mathrm{sph}, a}(r)}{d r}+f_{a}^{(2)}(\boldsymbol{r}) \frac{d^{2} \rho_{\mathrm{sph}, a}(r)}{d r^{2}}+O\left(\beta^{3}\right), \\
f_{a}^{(1)}(\boldsymbol{r})= & {\left[1+\left(2 \beta_{a}-\alpha_{a}\right)\right]\left(\beta_{a} \frac{x^{2}+y^{2}}{r}-\alpha_{a} \frac{z^{2}}{r}\right) } \\
& +\beta_{a}^{2} \frac{x^{2}+y^{2}}{r}+\alpha_{a}^{2} \frac{z^{2}}{r}-\frac{\beta_{a}^{2}}{2} \frac{x^{4}+y^{4}}{r^{3}}-\frac{\alpha_{a}^{2} z^{4}}{2}-\beta_{a}^{2} \frac{x^{2} y^{2}}{r^{3}}+\alpha_{a} \beta_{a} \frac{\left(x^{2}+y^{2}\right) z^{2}}{r^{3}}, \\
f_{a}^{(2)}(\boldsymbol{r})= & \frac{\beta_{a}^{2}}{2} \frac{x^{4}+y^{4}}{r^{2}}+\frac{\alpha_{a}^{2}}{2} \frac{z^{4}}{r^{2}}+\beta_{a}^{2} \frac{x^{2} y^{2}}{r^{2}}-\alpha_{a} \beta_{a} \frac{\left(x^{2}+y^{2}\right) z^{2}}{r^{2}} .
\end{aligned}
$$

The angular integration of $f_{a}^{(1)}$ and $f_{a}^{(2)}$ can be performed analytically as

$$
\begin{aligned}
& F_{a}^{(1)}(r):=\int f_{a}^{(1)}(\boldsymbol{r}) d \Omega=\frac{2 \pi}{15} r\left(17 \alpha_{a}^{2}-36 \alpha_{a} \beta_{a}+52 \beta_{a}^{2}-10 \alpha_{a}+20 \beta_{a}\right), \\
& F_{a}^{(2)}(r):=\int f_{a}^{(2)}(\boldsymbol{r}) d \Omega=\frac{2 \pi}{15} r^{2}\left(3 \alpha_{a}^{2}-4 \alpha_{a} \beta_{a}+8 \beta_{a}^{2}\right),
\end{aligned}
$$

respectively. Let us consider using this transformation how the energy of the system changes when an infinitesimal quadrupole deformation $\left|\alpha_{a}\right| \ll 1$ and $\left|\beta_{a}\right| \ll 1$ is induced to a spherically symmetric system $\rho_{\text {sph }}(\boldsymbol{r})=\rho_{\text {sph }}(r)$.

Kinetic energy: The kinetic energy reads

$$
\begin{aligned}
T_{\text {def }, a} & =\left\langle\Psi_{\text {def }, a}|\hat{T}| \Psi_{\text {def }, a}\right\rangle \\
& =\left(\frac{2}{3} e^{2 \beta_{a}}+\frac{1}{3} e^{-2 \alpha_{a}}\right)\left\langle\Psi_{\mathrm{sph}, a}|\hat{T}| \Psi_{\mathrm{sph}, a}\right\rangle \\
& =\left(\frac{2}{3} e^{2 \beta_{a}}+\frac{1}{3} e^{-2 \alpha_{a}}\right) T_{\mathrm{sph}, a} .
\end{aligned}
$$

Central external potential energy: By using $(B .3)$ and the angular integral 
(B.6) and (B.7), the central external potential changes as

$$
\begin{aligned}
& \Delta E_{\mathrm{ext}, a} \\
\simeq & {\left[\left(2 \beta_{a}-\alpha_{a}\right)+\frac{1}{2}\left(2 \beta_{a}-\alpha_{a}\right)^{2}\right] \int \rho_{\mathrm{sph}, a}(r) V_{\mathrm{ext}}(r) d \boldsymbol{r} } \\
& +\int f_{a}^{(1)}(\boldsymbol{r}) \frac{d \rho_{\mathrm{sph}, a}(r)}{d r} V_{\mathrm{ext}}(r) d \boldsymbol{r}+\int f_{a}^{(2)}(\boldsymbol{r}) \frac{d^{2} \rho_{\mathrm{sph}, a}(r)}{d r^{2}} V_{\mathrm{ext}}(r) d \boldsymbol{r} \\
= & 4 \pi\left[\left(2 \beta_{a}-\alpha_{a}\right)+\frac{1}{2}\left(2 \beta_{a}-\alpha_{a}\right)^{2}\right] \int_{0}^{\infty} \rho_{\mathrm{sph}, a}(r) V_{\mathrm{ext}}(r) r^{2} d r \\
& +\int_{0}^{\infty} F_{a}^{(1)}(r) \frac{d \rho_{\mathrm{sph}, a}(r)}{d r} V_{\mathrm{ext}}(r) r^{2} d r+\int_{0}^{\infty} F_{a}^{(2)}(r) \frac{d^{2} \rho_{\mathrm{sph}, a}(r)}{d r^{2}} V_{\mathrm{ext}}(r) r^{2} d r \\
= & 4 \pi\left[\left(2 \beta_{a}-\alpha_{a}\right)+\frac{1}{2}\left(2 \beta_{a}-\alpha_{a}\right)^{2}\right] \int_{0}^{\infty} \rho_{\mathrm{sph}, a}(r) V_{\mathrm{ext}}(r) r^{2} d r \\
& -\int_{0}^{\infty} \rho_{\mathrm{sph}, a}(r) \frac{d}{d r}\left[F_{a}^{(1)}(r) V_{\mathrm{ext}}(r) r^{2}\right] d r+\int_{0}^{\infty} \rho_{\mathrm{sph}, a}(r) \frac{d^{2}}{d r^{2}}\left[F_{a}^{(2)}(r) V_{\mathrm{ext}}(r) r^{2}\right] d r,
\end{aligned}
$$

where the external potential $V_{\text {ext }}(r) \sim r^{d}(d>-3)$ is assumed to behave as $r \rightarrow 0$. We assume the form of the external potential as $V_{\mathrm{ext}}(r)=c r^{d}$ with given constants $c$ and $d$. Substituting this form into $(\mathrm{B} .9)$, we get

$$
\begin{aligned}
\Delta E_{\mathrm{ext}, a}= & {\left[-\left(2 \beta_{a}-\alpha_{a}\right)+\frac{1}{2}\left(2 \beta_{a}-\alpha_{a}\right)^{2}\right.} \\
& -\frac{1}{30}(d+3)\left(17 \alpha_{a}^{2}-36 \alpha_{a} \beta_{a}+52 \beta_{a}^{2}-10 \alpha_{a}+20 \beta_{a}\right) \\
& \left.+\frac{1}{30}(d+4)(d+3)\left(3 \alpha_{a}^{2}-4 \alpha_{a} \beta_{a}+8 \beta_{a}^{2}\right)\right] E_{\mathrm{ext}, a}^{\mathrm{sph}} .
\end{aligned}
$$

In the case of the Coulomb potential $(d=-1)$, B.10 reads

$$
\begin{aligned}
& \Delta E_{\mathrm{ext}, a} \\
= & {\left[-\left(2 \beta_{a}-\alpha_{a}\right)+\frac{1}{2}\left(2 \beta_{a}-\alpha_{a}\right)^{2}-\frac{1}{15}\left(8 \alpha_{a}^{2}-24 \alpha_{a} \beta_{a}+28 \beta_{a}^{2}-10 \alpha_{a}+20 \beta_{a}\right)\right] E_{\mathrm{ext}, a}^{\mathrm{sph},} }
\end{aligned}
$$

while in the case of the harmonic oscillator potential $(d=2),(\mathrm{B} .10)$ reads

$$
\begin{aligned}
& \Delta E_{\mathrm{ext}, a} \\
= & {\left[-\left(2 \beta_{a}-\alpha_{a}\right)+\frac{1}{2}\left(2 \beta_{a}-\alpha_{a}\right)^{2}+\frac{1}{6}\left(\alpha_{a}^{2}+12 \alpha_{a} \beta_{a}-4 \beta_{a}^{2}-10 \alpha_{a}+20 \beta_{a}\right)\right] E_{\mathrm{ext}, a}^{\mathrm{sph}} . }
\end{aligned}
$$


Interaction energy: Substituting $(\mathrm{B} .3)$ and 44 into 43 , one obtains

$$
\begin{aligned}
E_{\mathrm{int}, a b}^{\mathrm{def}} \simeq & c_{a} c_{b} E_{\mathrm{int}, a b}^{\mathrm{sph}} \\
& +\frac{c_{a}}{2} \int_{0}^{\infty} \int_{0}^{\infty} F_{b}^{(1)}\left(r^{\prime}\right) \rho_{\mathrm{sph}, a}(r) \frac{d \rho_{\mathrm{sph}, b}\left(r^{\prime}\right)}{d r^{\prime}} V_{0}^{a b}\left(r, r^{\prime}\right) r^{2} r^{\prime 2} d r d r^{\prime} \\
& +\frac{c_{b}}{2} \int_{0}^{\infty} \int_{0}^{\infty} F_{a}^{(1)}(r) \frac{d \rho_{\mathrm{sph}, a}(r)}{d r} \rho_{\mathrm{sph}, b}\left(r^{\prime}\right) V_{0}^{a b}\left(r, r^{\prime}\right) r^{2} r^{\prime 2} d r d r^{\prime} \\
& +\frac{c_{a}}{2} \int_{0}^{\infty} \int_{0}^{\infty} F_{b}^{(2)}\left(r^{\prime}\right) \rho_{\mathrm{sph}, a}(r) \frac{d^{2} \rho_{\mathrm{sph}, b}\left(r^{\prime}\right)}{d r^{\prime 2}} V_{0}^{a b}\left(r, r^{\prime}\right) r^{2} r^{\prime 2} d r d r^{\prime} \\
& +\frac{c_{b}}{2} \int_{0}^{\infty} \int_{0}^{\infty} F_{a}^{(2)}(r) \frac{d^{2} \rho_{\mathrm{sph}, a}(r)}{d r^{2}} \rho_{\mathrm{sph}, b}\left(r^{\prime}\right) V_{0}^{a b}\left(r, r^{\prime}\right) r^{2} r^{\prime 2} d r d r^{\prime} \\
& +\frac{1}{8 \pi} \int_{0}^{\infty} \int_{0}^{\infty} F_{a}^{(1)}(r) F_{b}^{(1)}\left(r^{\prime}\right) \frac{d \rho_{\mathrm{sph}, a}(r)}{d r} \frac{d \rho_{\mathrm{sph}, b}\left(r^{\prime}\right)}{d r^{\prime}} V_{0}^{a b}\left(r, r^{\prime}\right) r^{2} r^{\prime 2} d r d r^{\prime} \\
& +\frac{8 \pi}{45}\left(\alpha_{a}+\beta_{a}\right)\left(\alpha_{b}+\beta_{b}\right) \int_{0}^{\infty} \int_{0}^{\infty} \frac{d \rho_{\mathrm{sph}, a}(r)}{d r} \frac{d \rho_{\mathrm{sph}, b}\left(r^{\prime}\right)}{d r^{\prime}} V_{2}^{a b}\left(r, r^{\prime}\right) r^{3} r^{\prime 3} d r d r^{\prime},
\end{aligned}
$$

where $c_{a}=1+\left(2 \beta_{a}-\alpha_{a}\right)+\frac{1}{2}\left(2 \beta_{a}-\alpha_{a}\right)^{2}$.

In the case of atomic nuclei, substituting 49 into B.13), one obtains

$$
\begin{aligned}
E_{\mathrm{int}, a b}^{\mathrm{def}} \simeq & c_{a} c_{b} E_{\mathrm{int}, a b}^{\mathrm{sph}} \\
& -\frac{\pi c_{a} g_{a b}}{15}\left(17 \alpha_{b}^{2}-36 \alpha_{b} \beta_{b}+52 \beta_{b}^{2}-10 \alpha_{b}+20 \beta_{b}\right) \int_{0}^{\infty} \rho_{\mathrm{sph}, a}(r) \frac{d \rho_{\mathrm{sph}, b}(r)}{d r} r^{3} d r \\
& -\frac{\pi c_{b} g_{a b}}{15}\left(17 \alpha_{a}^{2}-36 \alpha_{a} \beta_{a}+52 \beta_{a}^{2}-10 \alpha_{a}+20 \beta_{a}\right) \int_{0}^{\infty} \rho_{\mathrm{sph}, b}(r) \frac{d \rho_{\mathrm{sph}, a}(r)}{d r} r^{3} d r \\
& -\frac{\pi c_{a} g_{a b}}{15}\left(3 \alpha_{b}^{2}-4 \alpha_{b} \beta_{b}+8 \beta_{b}^{2}\right) \int_{0}^{\infty} \rho_{\mathrm{sph}, a}(r) \frac{d^{2} \rho_{\mathrm{sph}, b}(r)}{d r^{2}} r^{4} d r \\
& -\frac{\pi c_{b} g_{a b}}{15}\left(3 \alpha_{a}^{2}-4 \alpha_{a} \beta_{a}+8 \beta_{a}^{2}\right) \int_{0}^{\infty} \rho_{\mathrm{sph}, b}(r) \frac{d^{2} \rho_{\mathrm{sph}, a}(r)}{d r^{2}} r^{4} d r \\
& -\frac{\pi g_{a b}}{450}\left(17 \alpha_{a}^{2}-36 \alpha_{a} \beta_{a}+52 \beta_{a}^{2}-10 \alpha_{a}+20 \beta_{a}\right) \\
& \times\left(17 \alpha_{b}^{2}-36 \alpha_{b} \beta_{b}+52 \beta_{b}^{2}-10 \alpha_{b}+20 \beta_{b}\right) \int_{0}^{\infty} \frac{d \rho_{\mathrm{sph}, a}(r)}{d r} \frac{d \rho_{\mathrm{sph}, b}(r)}{d r} r^{4} d r \\
& -\frac{8 \pi g_{a b}}{45}\left(\alpha_{a}+\beta_{a}\right)\left(\alpha_{b}+\beta_{b}\right) \int_{0}^{\infty} \frac{d \rho_{\mathrm{sph}, a}(r)}{d r} \frac{d \rho_{\mathrm{sph}, b}(r)}{d r} r^{4} d r .
\end{aligned}
$$


In the case of atoms, substituting (51) into $\mathrm{B} .13$, one obtains

$$
\begin{aligned}
E_{\mathrm{int}, a b}^{\mathrm{def}} \simeq & c_{a} c_{b} E_{\mathrm{int}, a b}^{\mathrm{sph}} \\
+ & \frac{4 \pi^{2} c_{a}}{15}\left(17 \alpha_{b}^{2}-36 \alpha_{b} \beta_{b}+52 \beta_{b}^{2}-10 \alpha_{b}+20 \beta_{b}\right) \\
\quad & \quad \int_{0}^{\infty} \int_{0}^{\infty} \rho_{\mathrm{sph}, a}(r) \frac{d \rho_{\mathrm{sph}, b}\left(r^{\prime}\right)}{d r^{\prime}} \frac{1}{r_{>}} r^{2} r^{\prime 3} d r d r^{\prime} \\
+ & \frac{4 \pi^{2} c_{b}}{15}\left(17 \alpha_{a}^{2}-36 \alpha_{a} \beta_{a}+52 \beta_{a}^{2}-10 \alpha_{a}+20 \beta_{a}\right) \\
& \times \int_{0}^{\infty} \int_{0}^{\infty} \frac{d \rho_{\mathrm{sph}, a}(r)}{d r} \rho_{\mathrm{sph}, b}\left(r^{\prime}\right) \frac{1}{r_{>}} r^{3} r^{\prime 2} d r d r^{\prime} \\
+ & \frac{4 \pi^{2} c_{a}}{15}\left(3 \alpha_{b}^{2}-4 \alpha_{b} \beta_{b}+8 \beta_{b}^{2}\right) \int_{0}^{\infty} \int_{0}^{\infty} \rho_{\mathrm{sph}, a}(r) \frac{d^{2} \rho_{\mathrm{sph}, b}\left(r^{\prime}\right)}{d r^{\prime 2}} \frac{1}{r_{>}} r^{2} r^{\prime 4} d r d r^{\prime} \\
+ & \frac{4 \pi^{2} c_{b}}{15}\left(3 \alpha_{a}^{2}-4 \alpha_{a} \beta_{a}+8 \beta_{a}^{2}\right) \int_{0}^{\infty} \int_{0}^{\infty} \frac{d^{2} \rho_{\mathrm{sph}, a}(r)}{d r^{2}} \rho_{\mathrm{sph}, b}\left(r^{\prime}\right) \frac{1}{r_{>}} r^{4} r^{\prime 2} d r d r^{\prime} \\
+ & \frac{2 \pi^{2}}{225}\left(17 \alpha_{a}^{2}-36 \alpha_{a} \beta_{a}+52 \beta_{a}^{2}-10 \alpha_{a}+20 \beta_{a}\right) \\
\quad \times\left(17 \alpha_{b}^{2}-36 \alpha_{b} \beta_{b}+52 \beta_{b}^{2}-10 \alpha_{b}+20 \beta_{b}\right) & \times \int_{0}^{\infty} \int_{0}^{\infty} \frac{d \rho_{\mathrm{sph}, a}(r)}{d r} \frac{d \rho_{\mathrm{sph}, b}\left(r^{\prime}\right)}{d r^{\prime}} \frac{1}{r_{>}} r^{3} r^{\prime 3} d r d r^{\prime} \\
+ & \frac{32 \pi^{2}}{225}\left(\alpha_{a}+\beta_{a}\right)\left(\alpha_{b}+\beta_{b}\right) \int_{0}^{\infty} \int_{0}^{\infty} \frac{d \rho_{\mathrm{sph}, a}(r)}{d r} \frac{d \rho_{\mathrm{sph}, b}\left(r^{\prime}\right)}{d r^{\prime}} \frac{r_{<}^{2}}{r_{>}^{3}} r^{3} r^{\prime 3} d r d r^{\prime} .
\end{aligned}
$$

Substituting $\alpha_{a}=2 \beta_{a}$ into equations shown here, one can obtain equations shown in section 4. As long as the small deformation is considered, the conclusion shown in section 4 remains.

\section{Appendix C. Numerical data}

All the numerical data related to the main part of the paper are shown in Tables C1 C10. 
On deformability of atoms

Table C1. The total energies, the root-mean-square radii, $Z\left\langle r^{2}\right\rangle$, the quadrupole moments, $Q$, and the deformation parameters, $\beta$, calculated with the unrestricted Hartree-Fock method with the 6-31+G basis. Configurations of the valence electrons calculated by the natural orbital analysis are also shown.

The Hartree atomic unit is used for total energies, $Z\left\langle r^{2}\right\rangle$, and $Q$.

\begin{tabular}{|c|c|c|c|c|c|c|c|c|c|c|c|}
\hline Atom & $\mathrm{z}$ & Mult. & Energy & $z\left\langle r^{2}\right\rangle$ & $Q_{x}$ & $Q_{y}$ & $Q_{z}$ & $\beta_{x}$ & $\beta_{y}$ & $\beta_{z}$ & Config. \\
\hline $\mathrm{Li}$ & 3 & 2 & -7.4315 & 18.2800 & 0.0000 & 0.0000 & 0.0000 & 0.0000 & 0.0000 & 0.0000 & $2 s^{1.00}$ \\
\hline $\mathrm{Be}$ & 4 & 1 & -14.5696 & 17.3430 & 0.0000 & 0.0000 & 0.0000 & 0.0000 & 0.0000 & 0.0000 & $2 s^{2.00}$ \\
\hline $\mathrm{B}$ & 5 & 2 & -24.5237 & 15.9020 & -2.4718 & -2.4718 & +4.9435 & -0.1232 & -0.1232 & +0.2464 & $2 s^{2.00} 2 p^{1.00}$ \\
\hline $\mathrm{C}$ & 6 & 3 & -37.6809 & 13.8214 & +1.4877 & +1.4877 & -2.9754 & +0.0853 & +0.0853 & -0.1706 & $2 s^{2.00} 2 p^{2.00}$ \\
\hline $\mathrm{N}$ & 7 & 4 & -54.3863 & 12.1756 & 0.0000 & 0.0000 & 0.0000 & 0.0000 & 0.0000 & 0.0000 & $2 s^{2.00} 2 p^{3.00}$ \\
\hline $\mathrm{O}$ & 8 & 3 & -74.7835 & 11.3088 & -0.9747 & -0.9747 & $\begin{array}{r}0.0000 \\
+1.9496\end{array}$ & -0.0683 & -0.0683 & $\begin{array}{r}0.0000 \\
+0.1367\end{array}$ & $2 s^{2.00} 2 p^{3.99} 3 p^{0.01}$ \\
\hline $\mathrm{F}$ & 9 & 2 & -99.3675 & 10.3172 & +0.6952 & +0.6952 & -1.3904 & +0.0534 & +0.0534 & $\begin{array}{l}+0.1301 \\
-0.1068\end{array}$ & $2 s^{2.00} 2 p^{5.00}$ \\
\hline $\mathrm{Ne}$ & 10 & 1 & -128.4835 & 9.4321 & 0.0000 & 0.0000 & 0.0000 & 0.0000 & 0.0000 & 0.0000 & $2 s^{2.00} 2 p^{6.00}$ \\
\hline $\mathrm{Na}$ & 11 & 2 & -161.8414 & 27.1816 & 0.0000 & 0.0000 & 0.0000 & 0.0000 & 0.0000 & 0.0000 & $3 s^{1.00}$ \\
\hline $\mathrm{Mg}$ & 12 & 1 & -199.5953 & 29.6206 & 0.0000 & 0.0000 & 0.0000 & 0.0000 & 0.0000 & $\begin{array}{l}0.0000 \\
0.0000\end{array}$ & $3 s^{2.00}$ \\
\hline $\mathrm{Al}$ & 13 & 2 & -241.8545 & 33.4055 & -5.5694 & -5.5694 & +11.1387 & -0.1322 & -0.1322 & $\begin{array}{r}0.0000 \\
+0.2643\end{array}$ & $3 s^{2.00} 3 p^{1.00}$ \\
\hline $\mathrm{Si}$ & 14 & 3 & -288.8288 & 32.2974 & +3.5934 & +3.5934 & -7.1869 & +0.0882 & +0.0882 & -0.1764 & $3 s^{2.00} 3 p^{2.00}$ \\
\hline $\mathrm{P}$ & 15 & 4 & -340.6894 & 30.3267 & 0.0000 & 0.0000 & 0.0000 & 0.0000 & $\begin{array}{r}+0.0882 \\
0.0000\end{array}$ & $\begin{array}{r}-0.1704 \\
0.0000\end{array}$ & $3 s^{2.00} 3 p^{3.00}$ \\
\hline s & 16 & 3 & -397.4722 & 29.2999 & -2.3199 & -2.3199 & $\begin{array}{r}+4.6397 \\
\end{array}$ & -0.0628 & -0.0628 & +0.1255 & $3 s^{2.00} 3 p^{4.00}$ \\
\hline $\mathrm{Cl}$ & 17 & 2 & -459.4438 & 27.7141 & $\begin{array}{r}+1.7509 \\
+1.75\end{array}$ & $\begin{array}{r}1.015 \\
+1.7509\end{array}$ & -3.5020 & +0.0501 & $\begin{array}{l}-0.0020 \\
+0.0501\end{array}$ & $\begin{array}{l}+0.1200 \\
-0.1002\end{array}$ & $3 s^{2.00} 3 p^{5.00}$ \\
\hline Ar & 18 & 1 & -526.7731 & 26.0836 & 0.0000 & 0.0000 & 0.0000 & 0.0000 & 0.0000 & 0.0000 & $3 s^{2.00} 3 p^{6.00}$ \\
\hline $\mathrm{K}$ & 19 & 2 & -599.1190 & 51.2038 & 0.0000 & 0.0000 & 0.0000 & 0.0000 & 0.0000 & 0.0000 & $4 s^{1.00}$ \\
\hline $\mathrm{Ca}$ & 20 & 1 & -676.7079 & 56.6004 & 0.0000 & 0.0000 & 0.0000 & 0.0000 & 0.0000 & $\begin{array}{l}0.0000 \\
0.0000\end{array}$ & $4 s^{2.00}$ \\
\hline $\mathrm{Sc}$ & 21 & 2 & -759.6763 & 53.1310 & $\begin{array}{r}0.0000 \\
+0.2094\end{array}$ & $\begin{array}{r}0.0000 \\
+0.2094\end{array}$ & -0.4189 & $\begin{array}{r}0.0003 \\
+0.0031\end{array}$ & $\begin{array}{r}0.0000 \\
+0.0031\end{array}$ & $\begin{array}{r}0.0000 \\
-0.0062\end{array}$ & $4 s^{1.99} 3 d^{1.00}$ \\
\hline $\mathrm{Ti}$ & 22 & 3 & -848.3215 & 50.2245 & -0.1626 & -0.1626 & +0.3250 & -0.0026 & -0.0026 & +0.0051 & $4 s^{1.99} 3 d^{2.00}$ \\
\hline $\mathrm{V}$ & 23 & 4 & -942.7941 & 47.6799 & 0.0000 & 0.0000 & 0.0000 & 0.0000 & $\begin{array}{r}-0.0020 \\
0.0000\end{array}$ & $\begin{array}{r}+0.0051 \\
0.0000\end{array}$ & $4 s^{1.99} 3 d^{3.00} s^{0.01}$ \\
\hline $\mathrm{Cr}$ & 24 & 7 & -1043.2424 & 37.9685 & 0.0000 & 0.0000 & 0.0000 & 0.0000 & 0.0000 & 0.0000 & $4 s^{1.00} 3 d^{5.00}$ \\
\hline $\mathrm{Mn}$ & 25 & 6 & -1149.6148 & 37.0228 & +0.7490 & $\begin{array}{r}0.7490 \\
+0.749\end{array}$ & -1.4982 & +0.0160 & $\begin{array}{r}0.0000 \\
+0.0160\end{array}$ & -0.0321 & $4 s^{1.00} 3 d^{5.98} 4 d^{0.02}$ \\
\hline $\mathrm{Fe}$ & 26 & 5 & -1262.2043 & 35.7532 & -0.5400 & -0.5400 & +1.0797 & -0.0120 & -0.0120 & +0.0239 & $4 s^{1.00} 3 d^{6.99} 4 d^{0.01}$ \\
\hline $\mathrm{Co}$ & 27 & 4 & -1381.1643 & 34.5419 & 0.0000 & 0.0000 & 0.0000 & 0.0000 & 0.0000 & 0.0000 & $4 s^{1.00} 3 d^{8.00}$ \\
\hline $\mathrm{Ni}$ & 28 & 3 & -1506.5944 & 33.4315 & -0.4075 & -0.4075 & +0.8148 & -0.0097 & -0.0097 & $\begin{array}{r}0.0000 \\
+0.0193\end{array}$ & $4 s^{1.00} 3 d^{9.00}$ \\
\hline $\mathrm{Cu}$ & 29 & 2 & -1638.6938 & 32.2616 & 0.0000 & 0.0000 & $\begin{array}{r}+0.0140 \\
0.0000\end{array}$ & $\begin{array}{r}-0.0098 \\
0.0000\end{array}$ & $\begin{array}{r}-0.0091 \\
0.0000\end{array}$ & $\begin{array}{r}+0.0190 \\
0.0000\end{array}$ & $4 s^{1.00} 3 d^{10.00}$ \\
\hline $\mathrm{Zn}$ & 30 & 1 & $\begin{array}{r}-1050.0530 \\
-1777.5332\end{array}$ & 35.0221 & 0.0000 & 0.0000 & 0.0000 & 0.0000 & 0.0000 & $\begin{array}{l}0.0000 \\
0.0000\end{array}$ & $4 s^{2.00} 3 d^{10.00}$ \\
\hline $\mathrm{Ga}$ & 31 & 2 & -1921.1399 & 41.4244 & -5.5787 & -5.5787 & +11.1575 & -0.1068 & -0.1068 & $\begin{array}{r}0.0000 \\
+0.2135\end{array}$ & $4 s^{2.00} 4 p^{1.00}$ \\
\hline $\mathrm{Ge}$ & 32 & 3 & -2073.0829 & 42.1351 & +3.9175 & +3.9175 & -7.8353 & +0.0737 & +0.0737 & -0.1474 & $4 s^{2.00} 4 p^{2.00}$ \\
\hline $\mathrm{As}$ & 33 & 4 & -2231.8086 & 41.4771 & 0.0000 & 0.0000 & 0.0000 & 0.0000 & 0.0000 & 0.0000 & $4 s^{2.00} 4 p^{3.00}$ \\
\hline $\mathrm{Se}$ & 34 & 3 & -2397.3677 & 41.6200 & -2.8141 & -2.8141 & +5.6285 & -0.0536 & -0.0536 & +0.1072 & $4 s^{2.00} 4 p^{4.00}$ \\
\hline $\mathrm{Br}$ & 35 & 2 & -2569.7593 & 40.7722 & +2.2376 & +2.2376 & -4.4751 & +0.0435 & +0.0435 & -0.0870 & $4 s^{2.00} 4 p^{5.00}$ \\
\hline $\mathrm{Kr}$ & 36 & 1 & -2749.2009 & 39.7020 & 0.0000 & 0.0000 & 0.0000 & 0.0000 & 0.0000 & 0.0000 & $4 s^{2.00} 4 p^{6.00}$ \\
\hline
\end{tabular}


Table C2. Same as Table C1 but with the dAug-CC-pV5Z basis.

\begin{tabular}{|c|c|c|c|c|c|c|c|c|c|c|c|}
\hline Atom & Z & Mult. & Energy & $z\left\langle r^{2}\right\rangle$ & $Q_{x}$ & $Q_{y}$ & $Q_{z}$ & $\beta_{x}$ & $\beta_{y}$ & $\beta_{z}$ & Config. \\
\hline $\mathrm{Li}$ & 3 & 2 & -7.4327 & 18.6252 & 0.0000 & 0.0000 & 0.0000 & 0.0000 & 0.0000 & 0.0000 & $2 s^{1.00}$ \\
\hline $\mathrm{Be}$ & 4 & 1 & -14.5730 & 17.3210 & 0.0000 & 0.0000 & 0.0000 & 0.0000 & 0.0000 & 0.0000 & $2 s^{2.00}$ \\
\hline B & 5 & 2 & -24.5331 & 15.7731 & -2.4912 & -2.4912 & $\begin{array}{r}+.08023 \\
+4.9823\end{array}$ & -0.1252 & -0.1252 & $\begin{array}{r}0.0000 \\
+0.2504\end{array}$ & $2 s^{1.99} 2 p^{1.00} 3 d^{0.01}$ \\
\hline $\mathrm{C}$ & 6 & 3 & -37.6937 & 13.7574 & +1.4846 & +1.4846 & -2.9689 & +0.0855 & +0.0855 & $\begin{array}{l}+0.2504 \\
-0.1711\end{array}$ & $2 s^{1.99} 2 p^{2.00}$ \\
\hline $\mathrm{N}$ & 7 & 4 & -54.4045 & 12.0782 & 0.0000 & 0.0000 & 0.0000 & 0.0000 & 0.0000 & 0.0000 & $2 s^{2.00} 2 p^{3.00}$ \\
\hline $\mathrm{O}$ & 8 & 3 & -74.8188 & 11.2130 & -0.9517 & -0.9517 & +1.9037 & -0.0673 & -0.0673 & +0.1346 & $2 s^{2.00} 2 p^{3.99} 3 p^{0.01}$ \\
\hline $\mathrm{F}$ & 9 & 2 & -99.4161 & 10.2470 & +0.6738 & +0.6738 & -1.3476 & +0.0521 & +0.0521 & -0.1042 & $2 s^{2.00} 2 p^{5.00}$ \\
\hline $\mathrm{Ne}$ & 10 & 1 & -128.5468 & 9.3741 & 0.0000 & 0.0000 & 0.0000 & 0.0000 & 0.0000 & 0.0000 & $2 s^{2.00} 2 p^{6.00}$ \\
\hline $\mathrm{Na}$ & 11 & 2 & -161.8587 & 27.1413 & 0.0000 & 0.0000 & 0.0000 & 0.0000 & 0.0000 & 0.0000 & $3 s^{1.00}$ \\
\hline $\mathrm{Al}$ & 13 & 2 & -241.8807 & 33.1885 & -5.7634 & -5.7634 & +11.5268 & -0.1377 & -0.1377 & +0.2753 & $3 s^{1.99} 3 p^{1.00} 3 d^{0.01}$ \\
\hline $\mathrm{Si}$ & 14 & 3 & -288.8588 & 32.1436 & $\begin{array}{r}+3.6302 \\
\end{array}$ & $\begin{array}{r}-3.6302 \\
\end{array}$ & -7.2605 & +0.0895 & +0.0895 & $\begin{array}{l}+0.2153 \\
-0.1790\end{array}$ & $3 s^{1.99} 3 p^{2.00} 3 d^{0.01}$ \\
\hline $\mathrm{P}$ & 15 & 4 & -340.7192 & 30.2655 & 0.0000 & 0.0000 & 0.0000 & 0.0000 & 0.0000 & $\begin{array}{r}-0.1790 \\
0.0000\end{array}$ & $3 s^{2.00} 3 p^{3.00}$ \\
\hline s & 16 & 3 & -397.5132 & 29.1710 & -2.1695 & -2.1695 & +4.3391 & -0.0590 & -0.0590 & $\begin{array}{r}0.0000 \\
+0.1179\end{array}$ & $3 s^{1.99} 3 p^{4.00} 3 d^{0.01}$ \\
\hline $\mathrm{Cl}$ & 17 & 2 & -459.4897 & 27.6256 & $\begin{array}{l}+1.6258 \\
\end{array}$ & +1.6258 & -3.2517 & +0.0466 & +0.0466 & -0.0933 & $3 s^{2.00} 3 p^{5.00}$ \\
\hline $\mathrm{Ar}$ & 18 & 1 & -526.8173 & 26.0376 & 0.0000 & 0.0000 & 0.0000 & 0.0000 & 0.0000 & 0.0000 & $3 s^{2.00} 3 p^{6.00}$ \\
\hline $\mathrm{Sc}$ & 21 & 2 & -759.7408 & 53.1077 & +0.1976 & +0.1976 & -0.3955 & +0.0029 & +0.0029 & -0.0059 & $4 s^{1.99} 3 d^{1.00}$ \\
\hline $\mathrm{Ti}$ & 22 & 3 & -848.4026 & 50.2023 & -0.1508 & -0.1508 & +0.3016 & -0.0024 & -0.0024 & +0.0048 & $4 s^{2.00} 3 d^{2.00}$ \\
\hline $\mathrm{V}$ & 23 & 4 & -942.8935 & 47.6556 & 0.0000 & 0.0000 & 0.0000 & 0.0000 & 0.0000 & 0.0000 & $4 s^{1.99} 3 d^{3.00} s_{s}^{0.01}$ \\
\hline $\mathrm{Cr}$ & 24 & 7 & -1043.3567 & 37.6585 & 0.0000 & 0.0000 & 0.0000 & 0.0000 & 0.0000 & 0.0000 & $4 s^{1.00} 3 d^{5.00}$ \\
\hline Mn & 25 & 6 & -1149.7533 & 36.7555 & +0.6276 & +0.6276 & -1.2551 & +0.0135 & +0.0135 & -0.0271 & $4 s^{1.00} 3 d^{5.99} 4 d^{0.01}$ \\
\hline $\mathrm{Fe}$ & 26 & 5 & -1262.3743 & 35.4942 & -0.4318 & -0.4318 & +0.8636 & -0.0096 & -0.0096 & $\begin{array}{l}-0.0211 \\
+0.0193\end{array}$ & $4 s^{1.00} 3 d^{6.99} 4 d^{0.01}$ \\
\hline $\mathrm{Co}$ & 27 & 4 & -1381.3674 & 34.2853 & 0.0000 & 0.0000 & 0.0000 & 0.0000 & 0.0000 & 0.0000 & $4 s^{1.00} 3 d^{8.00}$ \\
\hline $\mathrm{Ni}$ & 28 & 3 & -1506.8305 & 33.1827 & +0.2806 & +0.3348 & -0.6154 & +0.0067 & +0.0080 & $\begin{array}{r}0.0000 \\
-0.0147\end{array}$ & $4 s^{1.00} 3 d^{9.00}$ \\
\hline $\mathrm{Cu}$ & 29 & 2 & -1638.9574 & 36.3225 & -0.0156 & $\begin{array}{l}+0.0340 \\
-0.2304\end{array}$ & +0.2462 & 0.0003 & -0.0050 & $\begin{array}{l}-0.0148 \\
+0.0054\end{array}$ & $4 s^{2.00} 3 d^{9.00}$ \\
\hline $\mathrm{Zn}$ & 30 & 1 & -1777.8480 & 34.9792 & 0.0000 & 0.0000 & 0.0000 & 0.0000 & 0.0000 & 0.0000 & $4 s^{2.00} 3 d^{10.00}$ \\
\hline $\mathrm{Ga}$ & 31 & 2 & -1923.2644 & 40.7328 & -5.6952 & -5.6952 & +11.3903 & -0.1108 & -0.1108 & +0.2217 & $4 s^{1.99} 4 p^{1.00} 4 d^{0.01}$ \\
\hline $\mathrm{Ge}$ & 32 & 3 & -2075.3638 & 41.5004 & +3.9233 & +3.9233 & -7.8466 & +0.0749 & +0.0749 & -0.1499 & $4 s^{1.99} 4 p^{2.00} 4 d^{0.01}$ \\
\hline As & 33 & 4 & -2234.2398 & 41.0402 & 0.0000 & 0.0000 & 0.0000 & 0.0000 & 0.0000 & 0.0000 & $4 s^{2.00} 4 p^{3.00}$ \\
\hline $\mathrm{Se}$ & 34 & 3 & -2399.8753 & 41.1769 & -2.5759 & -2.5759 & +5.1518 & -0.0496 & -0.0496 & +0.0992 & $4 s^{2.00} 4 p^{4.00}$ \\
\hline $\mathrm{Br}$ & 35 & 2 & -2572.4483 & 40.5058 & +2.0362 & +2.0362 & -4.0723 & +0.0398 & $\begin{array}{r}0.0398 \\
+0.0398\end{array}$ & -0.0797 & $4 s^{2.00} 4 p^{5.00}$ \\
\hline $\mathrm{Kr}$ & 36 & 1 & -2752.0549 & 39.5314 & 0.0000 & 0.0000 & 0.0000 & 0.0000 & 0.0000 & 0.0000 & $4 s^{2.00} 4 p^{6.00}$ \\
\hline
\end{tabular}


Table C3. Same as Table C1 but with the STO-3G basis.

\begin{tabular}{|c|c|c|c|c|c|c|c|c|c|c|c|}
\hline Atom & $\mathrm{z}$ & Mult. & Energy & $z\left\langle r^{2}\right\rangle$ & $Q_{x}$ & $Q_{y}$ & $Q_{z}$ & $\beta_{x}$ & $\beta_{y}$ & $\beta_{z}$ & Config. \\
\hline $\mathrm{Li}$ & 3 & 2 & -7.3155 & 13.0955 & 0.0000 & 0.0000 & 0.0000 & 0.0000 & 0.0000 & 0.0000 & $2 s^{1.00}$ \\
\hline $\mathrm{Be}$ & 4 & 1 & -14.3519 & 12.3657 & 0.0000 & 0.0000 & 0.0000 & 0.0000 & 0.0000 & 0.0000 & $2 s^{2.00}$ \\
\hline B & 5 & 2 & -24.1490 & 10.6497 & -1.3369 & -1.3369 & +2.6738 & -0.0995 & -0.0995 & +0.1990 & $2 s^{2.00} 2 p^{1.00}$ \\
\hline $\mathrm{C}$ & 6 & 3 & -37.1984 & 10.5788 & +1.0169 & +1.0169 & -2.0335 & +0.0762 & +0.0762 & -0.1524 & $2 s^{2.00} 2 p^{2.00}$ \\
\hline $\mathrm{N}$ & 7 & 4 & -53.7190 & 10.1785 & 0.0000 & 0.0000 & 0.0000 & 0.0000 & 0.0000 & 0.0000 & $2 s^{2.00} 2 p^{3.00}$ \\
\hline $\mathrm{O}$ & 8 & 3 & -73.8042 & 9.1334 & -0.5942 & -0.5942 & +1.1884 & -0.0516 & -0.0516 & +0.1031 & $2 s^{2.00} 2 p^{4.00}$ \\
\hline $\mathrm{F}$ & 9 & 2 & -97.9865 & 8.2689 & +0.4626 & +0.4626 & -0.9252 & +0.0443 & +0.0443 & -0.0887 & $2 s^{2.00} 2 p^{5.00}$ \\
\hline $\mathrm{Ne}$ & 10 & 1 & -126.6045 & 7.3940 & 0.0000 & 0.0000 & 0.0000 & 0.0000 & 0.0000 & 0.0000 & $2 s^{2.00} 2 p^{6.00}$ \\
\hline $\mathrm{Na}$ & 11 & 2 & -159.6684 & 12.1291 & 0.0000 & 0.0000 & 0.0000 & 0.0000 & 0.0000 & 0.0000 & $3 s^{1.00}$ \\
\hline $\mathrm{Mg}$ & 12 & 1 & -197.0074 & 15.6258 & 0.0000 & 0.0000 & 0.0000 & 0.0000 & 0.0000 & 0.0000 & $3 s^{2.00}$ \\
\hline $\mathrm{Al}$ & 13 & 2 & -238.8584 & 19.1823 & -2.0286 & -2.0286 & +4.0571 & -0.0838 & -0.0838 & +0.1677 & $3 s^{2.00} 3 p^{1.00}$ \\
\hline $\mathrm{Si}$ & 14 & 3 & -285.4662 & 22.0780 & +1.9050 & +1.9050 & -3.8102 & +0.0684 & +0.0684 & -0.1368 & $3 s^{2.00} 3 p^{2.00}$ \\
\hline $\mathrm{P}$ & 15 & 4 & -336.8688 & 22.6495 & 0.0000 & 0.0000 & 0.0000 & 0.0000 & 0.0000 & 0.0000 & $3 s^{2.00} 3 p^{3.00}$ \\
\hline $\mathrm{S}$ & 16 & 3 & -393.1302 & 22.8523 & -1.3907 & -1.3907 & +2.7811 & -0.0482 & -0.0482 & +0.0965 & $3 s^{2.00} 3 p^{4.00}$ \\
\hline $\mathrm{Cl}$ & 17 & 2 & -454.5422 & 24.7260 & +1.3180 & +1.3180 & -2.6361 & +0.0423 & +0.0423 & -0.0845 & $3 s^{2.00} 3 p^{5.00}$ \\
\hline $\mathrm{Ar}$ & 18 & 1 & -521.2229 & 22.9344 & 0.0000 & 0.0000 & 0.0000 & 0.0000 & 0.0000 & 0.0000 & $3 s^{2.00} 3 p^{6.00}$ \\
\hline K & 19 & 2 & -593.0778 & 33.1098 & 0.0000 & 0.0000 & 0.0000 & 0.0000 & 0.0000 & 0.0000 & $4 s^{1.00}$ \\
\hline $\mathrm{Ca}$ & 20 & 1 & -669.9889 & 42.2174 & 0.0000 & 0.0000 & 0.0000 & 0.0000 & 0.0000 & 0.0000 & $4 s^{2.00}$ \\
\hline Sc & 21 & 2 & -751.9936 & 44.3275 & +2.4323 & +2.4323 & -4.8643 & +0.0435 & +0.0435 & -0.0870 & $4 s^{1.98} 3 d^{1.02}$ \\
\hline $\mathrm{Ti}$ & 22 & 3 & -839.5552 & 36.4583 & -0.8418 & -0.8418 & +1.6833 & -0.0183 & -0.0183 & +0.0366 & $4 s^{1.99} 3 d^{2.01}$ \\
\hline V & 23 & 4 & -932.3822 & 51.8131 & 0.0000 & 0.0000 & 0.0000 & 0.0000 & 0.0000 & 0.0000 & $4 s^{2.00} 4 p^{3.00}$ \\
\hline $\mathrm{Cr}$ & 24 & 7 & -1031.9037 & 43.3117 & -0.4164 & -0.4164 & +0.8326 & -0.0076 & -0.0076 & +0.0152 & $4 s^{1.00} 3 d^{2.00} 4 p^{3.00}$ \\
\hline $\mathrm{Mn}$ & 25 & 6 & -1137.6484 & 31.6207 & 0.0000 & 0.0000 & 0.0000 & 0.0000 & 0.0000 & 0.0000 & $4 s^{2.00} 3 d^{5.00}$ \\
\hline $\mathrm{Fe}$ & 26 & 5 & -1249.0414 & 41.1316 & -3.8513 & -3.8513 & +7.7028 & -0.0742 & -0.0742 & +0.1484 & $4 s^{2.00} 3 d^{5.00} 4 p^{1.00}$ \\
\hline Co & 27 & 4 & -1366.3895 & 48.9607 & +3.8432 & +3.8432 & -7.6865 & +0.0622 & +0.0622 & -0.1244 & $4 s^{2.00} 3 d^{5.00} 4 p^{2.00}$ \\
\hline $\mathrm{Ni}$ & 28 & 3 & -1490.1299 & 46.3092 & +3.8145 & +3.8145 & -7.6292 & +0.0653 & +0.0653 & -0.1306 & $4 s^{2.00} 3 d^{6.00} 4 p^{2.00}$ \\
\hline $\mathrm{Cu}$ & 29 & 2 & -1620.3878 & 37.6504 & -3.5702 & -3.5702 & +7.1405 & -0.0752 & -0.0752 & +0.1503 & $4 s^{2.00} 3 d^{8.00} 4 p^{1.00}$ \\
\hline $\mathrm{Zn}$ & 30 & 1 & -1757.1763 & 35.4563 & -4.8110 & -4.8110 & +9.6221 & -0.1076 & -0.1076 & +0.2151 & $4 s^{2.00} 3 d^{8.00} 4 p^{2.00}$ \\
\hline $\mathrm{Ga}$ & 31 & 2 & -1900.7285 & 31.3321 & -2.8420 & -2.8420 & +5.6842 & -0.0719 & -0.0719 & +0.1438 & $4 s^{2.00} 4 p^{1.00}$ \\
\hline $\mathrm{Ge}$ & 32 & 3 & -2051.6363 & 32.0788 & +2.3143 & +2.3143 & -4.6288 & +0.0572 & +0.0572 & -0.1144 & $4 s^{2.00} 4 p^{2.00}$ \\
\hline As & 33 & 4 & -2209.2637 & 33.7744 & 0.0000 & 0.0000 & 0.0000 & 0.0000 & 0.0000 & 0.0000 & $4 s^{2.00} 4 p^{3.00}$ \\
\hline $\mathrm{Se}$ & 34 & 3 & -2373.5273 & 35.4117 & -1.8814 & -1.8814 & +3.7627 & -0.0421 & -0.0421 & +0.0842 & $4 s^{2.00} 4 p^{4.00}$ \\
\hline $\mathrm{Br}$ & 35 & 2 & -2544.6368 & 35.2509 & +1.6414 & +1.6414 & -3.2825 & +0.0369 & +0.0369 & -0.0738 & $4 s^{2.00} 4 p^{5.00}$ \\
\hline $\mathrm{Kr}$ & 36 & 1 & -2722.7060 & 34.8481 & 0.0000 & 0.0000 & 0.0000 & 0.0000 & 0.0000 & 0.0000 & $4 s^{2.00} 4 p^{6.00}$ \\
\hline $\mathrm{Rb}$ & 37 & 2 & -2907.6043 & 44.3734 & 0.0000 & 0.0000 & 0.0000 & 0.0000 & 0.0000 & 0.0000 & $5 s^{1.00}$ \\
\hline $\mathrm{Sr}$ & 38 & 1 & -3099.1167 & 51.3762 & 0.0000 & 0.0000 & 0.0000 & 0.0000 & 0.0000 & 0.0000 & $5 s^{2.00}$ \\
\hline Y & 39 & 2 & -3297.3418 & 58.4961 & +0.9769 & +0.9769 & -1.9539 & +0.0132 & +0.0132 & -0.0265 & $5 s^{1.72} 4 d^{1.28}$ \\
\hline $\mathrm{Zr}$ & 40 & 3 & -3503.0360 & 53.2014 & -1.2644 & -1.2644 & +2.5289 & -0.0188 & -0.0188 & +0.0377 & $5 s^{1.81} 4 d^{2.19}$ \\
\hline $\mathrm{Nb}$ & 41 & 6 & -3715.8900 & 45.9028 & +1.1380 & +1.1380 & -2.2759 & +0.0197 & +0.0197 & -0.0393 & $5 s^{1.00} 4 d^{4.00}$ \\
\hline Mo & 42 & 7 & -3935.8966 & 43.4772 & 0.0000 & 0.0000 & 0.0000 & 0.0000 & 0.0000 & 0.0000 & $5 s^{1.00} 4 d^{5.00}$ \\
\hline $\mathrm{Tc}$ & 43 & 6 & -4163.2052 & 49.5440 & 0.0000 & 0.0000 & 0.0000 & 0.0000 & 0.0000 & 0.0000 & $5 s^{2.00} 4 d^{5.00}$ \\
\hline $\mathrm{Ru}$ & 44 & 5 & -4397.7215 & 50.7468 & +0.6444 & +0.6444 & -1.2887 & +0.0101 & +0.0101 & -0.0201 & $5 s^{2.00} 4 d^{6.00}$ \\
\hline $\mathrm{Rh}$ & 45 & 4 & -4639.7207 & 49.6014 & -0.5554 & -0.5554 & +1.1108 & -0.0089 & -0.0089 & +0.0178 & $5 s^{2.00} 4 d^{7.00}$ \\
\hline $\mathrm{Pd}$ & 46 & 1 & -4889.1921 & 48.0030 & +0.9705 & +0.9705 & -1.9409 & +0.0160 & +0.0160 & -0.0321 & $5 s^{2.00} 4 d^{8.00}$ \\
\hline $\mathrm{Ag}$ & 47 & 2 & -5146.5464 & 47.5080 & -0.6013 & -0.3270 & +0.9283 & -0.0100 & -0.0055 & +0.0155 & $5 s^{2.00} 4 d^{9.00}$ \\
\hline $\mathrm{Cd}$ & 48 & 1 & -5411.5327 & 41.7214 & 0.0000 & 0.0000 & 0.0000 & 0.0000 & 0.0000 & 0.0000 & $5 s^{2.00} 4 d^{10.00}$ \\
\hline In & 49 & 2 & -5682.7774 & 47.0137 & -3.2899 & -3.2899 & +6.5798 & -0.0555 & -0.0555 & +0.1109 & $5 s^{2.00} 5 p^{1.00}$ \\
\hline Sn & 50 & 3 & -5963.2061 & 50.2820 & +2.9919 & +2.9919 & -5.9838 & +0.0472 & +0.0472 & -0.0943 & $5 s^{2.00} 5 p^{2.00}$ \\
\hline $\mathrm{Sb}$ & 51 & 4 & -6251.3625 & 54.2873 & 0.0000 & 0.0000 & 0.0000 & 0.0000 & 0.0000 & 0.0000 & $5 s^{2.00} 5 p^{3.00}$ \\
\hline $\mathrm{Te}$ & 52 & 3 & -6547.1224 & 56.9144 & -2.6502 & -2.6502 & +5.3004 & -0.0369 & -0.0369 & +0.0738 & $5 s^{2.00} 5 p^{4.00}$ \\
\hline I & 53 & 2 & -6850.6762 & 57.2039 & +2.3607 & +2.3607 & -4.7214 & +0.0327 & +0.0327 & -0.0654 & $5 s^{2.00} 5 p^{5.00}$ \\
\hline $\mathrm{Xe}$ & 54 & 1 & -7162.1042 & 56.8048 & 0.0000 & 0.0000 & 0.0000 & 0.0000 & 0.0000 & 0.0000 & $5 s^{2.00} 5 p^{6.00}$ \\
\hline
\end{tabular}




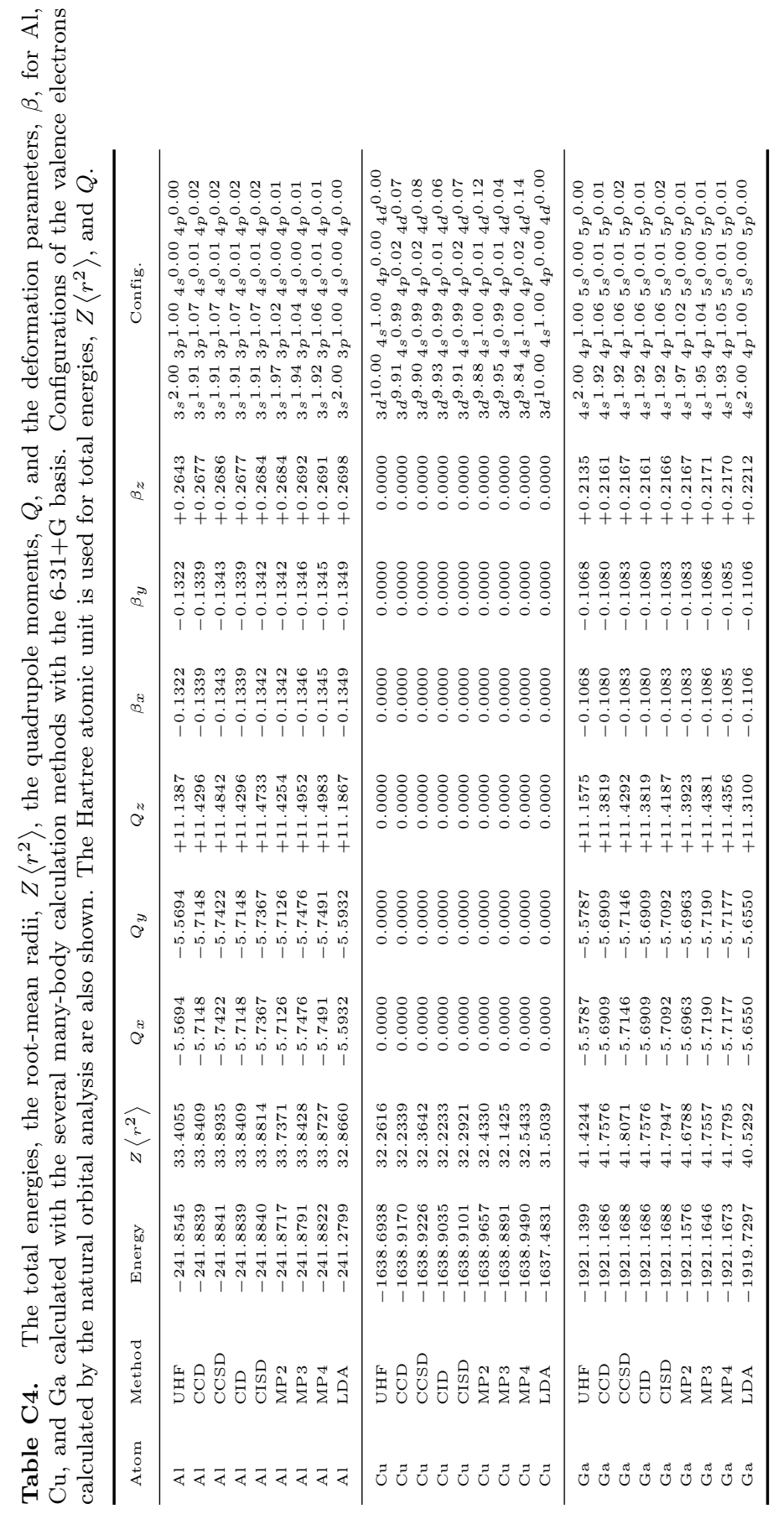




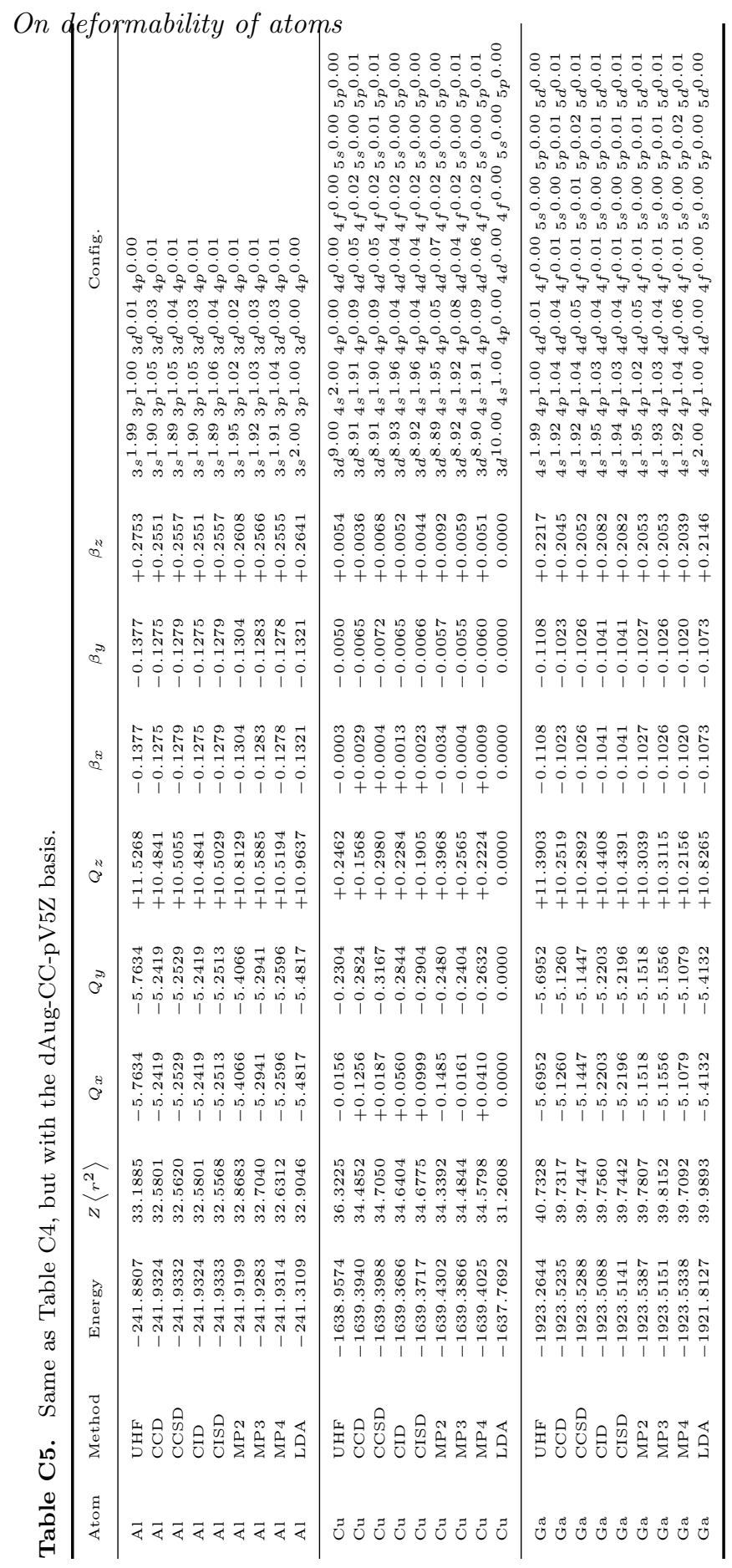


Table C6. The single-particle quadrupole moments $q_{i}^{(\mathrm{sp})}$ and the single-particle root-mean-square radii $\left\langle r^{2}\right\rangle_{n l}$ for the occupied orbitals in the $\mathrm{Al}$ atom calculated by the unrestricted Hartree-Fock method with the $6-31+\mathrm{G}$ basis. The singleparticle energy of each orbital, $\varepsilon_{j}$, and the corresponding orbital are also shown. The Hartree atomic unit is used for $\varepsilon_{j},\left\langle r^{2}\right\rangle_{n l}$, and $q_{z}^{(\mathrm{sp})}$.

\begin{tabular}{rlrrrrr}
\hline Spin & Orbital & \multicolumn{1}{c}{$\varepsilon_{j}$} & $\left\langle r^{2}\right\rangle_{n l}$ & \multicolumn{1}{c}{$q_{x}^{(\mathrm{sp})}$} & \multicolumn{1}{c}{$q_{y}^{(\mathrm{sp})}$} & \multicolumn{1}{c}{$q_{z}^{(\mathrm{sp})}$} \\
\hline$\alpha$ & $1 s$ & -58.4930 & 0.0195 & 0.0000 & 0.0000 & 0.0000 \\
$\alpha$ & $2 s$ & -4.9115 & 0.4591 & 0.0000 & 0.0000 & 0.0000 \\
$\alpha$ & $2 p_{z}$ & -3.2244 & 0.4535 & -0.1814 & -0.1814 & +0.3628 \\
$\alpha$ & $2 p_{x}$ & -3.2186 & 0.4561 & +0.3648 & -0.1824 & -0.1824 \\
$\alpha$ & $2 p_{y}$ & -3.2186 & 0.4561 & -0.1824 & +0.3648 & -0.1824 \\
$\alpha$ & $3 s$ & -0.4238 & 7.9466 & 0.0000 & 0.0000 & 0.0000 \\
$\alpha$ & $3 p_{z}$ & -0.2099 & 13.9269 & -5.5708 & -5.5708 & +11.1416 \\
\hline$\beta$ & $1 s$ & -58.4903 & 0.0195 & 0.0000 & 0.0000 & 0.0000 \\
$\beta$ & $2 s$ & -4.9081 & 0.4588 & 0.0000 & 0.0000 & 0.0000 \\
$\beta$ & $2 p_{x}$ & -3.2173 & 0.4557 & +0.3646 & -0.1823 & -0.1823 \\
$\beta$ & $2 p_{y}$ & -3.2173 & 0.4557 & -0.1823 & +0.3646 & -0.1823 \\
$\beta$ & $2 p_{z}$ & -3.2080 & 0.4547 & -0.1819 & -0.1819 & +0.3637 \\
$\beta$ & $3 s$ & -0.3628 & 7.8434 & 0.0000 & 0.0000 & 0.0000 \\
\hline Total & & 33.4055 & -5.5693 & -5.5693 & +11.1387 \\
\hline
\end{tabular}

Table C7. Same as Table C6 but for the Sc atom.

\begin{tabular}{rlrrrrr}
\hline Spin & Orbital & \multicolumn{1}{c}{$\varepsilon_{j}$} & $\left\langle r^{2}\right\rangle_{n l}$ & \multicolumn{1}{c}{$q_{x}^{(\mathrm{sp})}$} & \multicolumn{1}{c}{$q_{y}^{(\mathrm{sp})}$} & \multicolumn{1}{c}{$q_{z}^{(\mathrm{sp})}$} \\
\hline$\alpha$ & $1 s$ & -165.8952 & 0.0073 & 0.0000 & 0.0000 & 0.0000 \\
$\alpha$ & $2 s$ & -19.0956 & 0.1393 & 0.0000 & 0.0000 & 0.0000 \\
$\alpha$ & $2 p_{z}$ & -15.6861 & 0.1162 & -0.0465 & -0.0465 & +0.0930 \\
$\alpha$ & $2 p_{x}$ & -15.6830 & 0.1164 & +0.0931 & -0.0466 & -0.0466 \\
$\alpha$ & $2 p_{y}$ & -15.6830 & 0.1164 & -0.0466 & +0.0931 & -0.0466 \\
$\alpha$ & $3 s$ & -2.5988 & 1.3396 & +0.0016 & +0.0016 & -0.0031 \\
$\alpha$ & $3 p_{x}$ & -1.6232 & 1.6265 & +1.3012 & -0.6506 & -0.6506 \\
$\alpha$ & $3 p_{y}$ & -1.6232 & 1.6265 & -0.6506 & +1.3012 & -0.6506 \\
$\alpha$ & $3 p_{z}$ & -1.5893 & 1.6107 & -0.6443 & -0.6443 & +1.2886 \\
$\alpha$ & $3 d_{x y}$ & -0.3375 & 3.7873 & +1.0821 & +1.0821 & -2.1642 \\
$\alpha$ & $4 s$ & -0.2171 & 17.4198 & +0.1076 & +0.1076 & -0.2152 \\
\hline$\beta$ & $1 s$ & -165.8951 & 0.0073 & 0.0000 & 0.0000 & 0.0000 \\
$\beta$ & $2 s$ & -19.0832 & 0.1391 & -0.0001 & -0.0001 & +0.0001 \\
$\beta$ & $2 p_{z}$ & -15.6837 & 0.1162 & -0.0465 & -0.0465 & +0.0929 \\
$\beta$ & $2 p_{x}$ & -15.6657 & 0.1161 & +0.0929 & -0.0464 & -0.0464 \\
$\beta$ & $2 p_{y}$ & -15.6657 & 0.1161 & -0.0464 & +0.0929 & -0.0464 \\
$\beta$ & $3 s$ & -2.5451 & 1.3370 & -0.0194 & -0.0194 & +0.0387 \\
$\beta$ & $3 p_{z}$ & -1.5798 & 1.6156 & -0.6462 & -0.6462 & +1.2925 \\
$\beta$ & $3 p_{x}$ & -1.5273 & 1.6196 & +1.2957 & -0.6478 & -0.6478 \\
$\beta$ & $3 p_{y}$ & -1.5273 & 1.6196 & -0.6478 & +1.2957 & -0.6478 \\
$\beta$ & $4 s$ & -0.2051 & 18.5388 & -0.9703 & -0.9703 & +1.9406 \\
\hline Total & & & 53.1312 & +0.2095 & +0.2095 & -0.4189 \\
\hline
\end{tabular}


Table C8. Same as Table C6 but for the Ti atom.

\begin{tabular}{rlrrrrr}
\hline \multirow{2}{*}{ Spin } & Orbital & \multicolumn{1}{c}{$\varepsilon_{j}$} & $\left\langle r^{2}\right\rangle_{n l}$ & \multicolumn{1}{c}{$q_{x}^{(\mathrm{sp})}$} & \multicolumn{1}{c}{$q_{y}^{(\mathrm{sp})}$} & \multicolumn{1}{c}{$q_{z}^{(\mathrm{sp})}$} \\
\hline$\alpha$ & $1 s$ & -183.2759 & 0.0066 & 0.0000 & 0.0000 & 0.0000 \\
$\alpha$ & $2 s$ & -21.4572 & 0.1250 & 0.0000 & 0.0000 & 0.0000 \\
$\alpha$ & $2 p_{x}$ & -17.8273 & 0.1034 & +0.0827 & -0.0414 & -0.0414 \\
$\alpha$ & $2 p_{y}$ & -17.8273 & 0.1034 & -0.0414 & +0.0827 & -0.0414 \\
$\alpha$ & $2 p_{z}$ & -17.8244 & 0.1036 & -0.0414 & -0.0414 & +0.0829 \\
$\alpha$ & $3 s$ & -2.9463 & 1.1792 & -0.0016 & -0.0016 & +0.0032 \\
$\alpha$ & $3 p_{z}$ & -1.9071 & 1.4095 & -0.5638 & -0.5638 & +1.1276 \\
$\alpha$ & $3 p_{x}$ & -1.8680 & 1.4017 & +1.1213 & -0.5607 & -0.5607 \\
$\alpha$ & $3 p_{y}$ & -1.8680 & 1.4017 & -0.5607 & +1.1213 & -0.5607 \\
$\alpha$ & $3 d_{y z}$ & -0.4248 & 2.8534 & -1.6305 & +0.8153 & +0.8153 \\
$\alpha$ & $3 d_{z x}$ & -0.4248 & 2.8534 & +0.8153 & -1.6305 & +0.8153 \\
$\alpha$ & $4 s$ & -0.2338 & 15.4686 & +0.0616 & +0.0616 & -0.1232 \\
\hline$\beta$ & $1 s$ & -183.2754 & 0.0066 & 0.0000 & 0.0000 & 0.0000 \\
$\beta$ & $2 s$ & -21.4247 & 0.1247 & +0.0001 & +0.0001 & -0.0001 \\
$\beta$ & $2 p_{x}$ & -17.8015 & 0.1031 & +0.0825 & -0.0412 & -0.0412 \\
$\beta$ & $2 p_{y}$ & -17.8015 & 0.1031 & -0.0412 & +0.0825 & -0.0412 \\
$\beta$ & $2 p_{z}$ & -17.7791 & 0.1030 & -0.0412 & -0.0412 & +0.0824 \\
$\beta$ & $3 s$ & -2.8211 & 1.1778 & +0.0172 & +0.0172 & -0.0344 \\
$\beta$ & $3 p_{x}$ & -1.7472 & 1.4036 & +1.1229 & -0.5614 & -0.5614 \\
$\beta$ & $3 p_{y}$ & -1.7472 & 1.4036 & -0.5614 & +1.1229 & -0.5614 \\
$\beta$ & $3 p_{z}$ & -1.6867 & 1.4105 & -0.5642 & -0.5642 & +1.1284 \\
$\beta$ & $4 s$ & -0.2112 & 17.3797 & +0.5815 & +0.5815 & -1.1629 \\
\hline Total & & & 50.2249 & -0.1625 & -0.1625 & +0.3249 \\
\hline
\end{tabular}


On deformability of atoms

Table C9. Same as Table C6 but for the Ni atom.

\begin{tabular}{rlrrrrr}
\hline Spin & Orbital & \multicolumn{1}{c}{$\varepsilon_{j}$} & $\left\langle r^{2}\right\rangle_{n l}$ & $q_{x}^{(\mathrm{sp})}$ & \multicolumn{1}{c}{$q_{y}^{(\mathrm{sp})}$} & \multicolumn{1}{c}{$q_{z}^{(\mathrm{sp})}$} \\
\hline$\alpha$ & $1 s$ & -305.4079 & 0.0040 & 0.0000 & 0.0000 & 0.0000 \\
$\alpha$ & $2 s$ & -37.7247 & 0.0720 & 0.0000 & 0.0000 & -0.0001 \\
$\alpha$ & $2 p_{x}$ & -32.7657 & 0.0574 & +0.0459 & -0.0230 & -0.0230 \\
$\alpha$ & $2 p_{y}$ & -32.7657 & 0.0574 & -0.0230 & +0.0459 & -0.0230 \\
$\alpha$ & $2 p_{z}$ & -32.7281 & 0.0574 & -0.0229 & -0.0229 & +0.0459 \\
$\alpha$ & $3 s$ & -4.7265 & 0.6527 & +0.0073 & +0.0073 & -0.0147 \\
$\alpha$ & $3 p_{x}$ & -3.1649 & 0.7371 & +0.5897 & -0.2948 & -0.2948 \\
$\alpha$ & $3 p_{y}$ & -3.1649 & 0.7371 & -0.2948 & +0.5897 & -0.2948 \\
$\alpha$ & $3 p_{z}$ & -3.0843 & 0.7415 & -0.2966 & -0.2966 & +0.5932 \\
$\alpha$ & $3 d_{x} y^{2}$ & -0.5446 & 1.4257 & +0.4073 & +0.4073 & -0.8147 \\
$\alpha$ & $3 d_{x y}$ & -0.4962 & 1.5120 & +0.4320 & +0.4320 & -0.8640 \\
$\alpha$ & $3 d_{y z}$ & -0.4616 & 1.5739 & -0.8994 & +0.4497 & +0.4497 \\
$\alpha$ & $3 d_{z x}$ & -0.4616 & 1.5739 & +0.4497 & -0.8994 & +0.4497 \\
$\alpha$ & $3 d_{z^{2}}$ & -0.4493 & 1.5996 & -0.4427 & -0.4427 & +0.8854 \\
$\alpha$ & $4 s$ & -0.2395 & 13.2134 & +0.1360 & +0.1360 & -0.2720 \\
\hline$\beta$ & $1 s$ & -305.4054 & 0.0040 & 0.0000 & 0.0000 & 0.0000 \\
$\beta$ & $2 s$ & -37.6883 & 0.0719 & 0.0000 & 0.0000 & 0.0000 \\
$\beta$ & $2 p_{x}$ & -32.7198 & 0.0572 & +0.0458 & -0.0229 & -0.0229 \\
$\beta$ & $2 p_{y}$ & -32.7198 & 0.0572 & -0.0229 & +0.0458 & -0.0229 \\
$\beta$ & $2 p_{z}$ & -32.7194 & 0.0573 & -0.0229 & -0.0229 & +0.0459 \\
$\beta$ & $3 s$ & -4.6262 & 0.6549 & -0.0005 & -0.0005 & +0.0010 \\
$\beta$ & $3 p_{z}$ & -3.0570 & 0.7407 & -0.2963 & -0.2963 & +0.5926 \\
$\beta$ & $3 p_{x}$ & -3.0065 & 0.7383 & +0.5907 & -0.2953 & -0.2953 \\
$\beta$ & $3 p_{y}$ & -3.0065 & 0.7383 & -0.2953 & +0.5907 & -0.2953 \\
$\beta$ & $3 d_{x y}$ & -0.4702 & 1.4877 & +0.4251 & +0.4251 & -0.8501 \\
$\beta$ & $3 d_{y z}$ & -0.4160 & 1.5895 & -0.9083 & +0.4541 & +0.4541 \\
$\beta$ & $3 d_{z x}$ & -0.4160 & 1.5895 & +0.4541 & -0.9083 & +0.4541 \\
$\beta$ & $3 d_{z}$ & -0.3977 & 1.6301 & -0.4655 & -0.4655 & +0.9309 \\
\hline Total & & & 33.4318 & -0.4074 & -0.4074 & +0.8148 \\
\hline & & & & & & \\
\hline
\end{tabular}


Table C10. Same as Table C6 but for the Ga atom.

\begin{tabular}{|c|c|c|c|c|c|c|}
\hline Spin & Orbital & $\varepsilon_{j}$ & $\left\langle r^{2}\right\rangle_{n l}$ & $q_{x}^{(\mathrm{sp})}$ & $q_{y}^{(\mathrm{sp})}$ & $q_{z}^{(\mathrm{sp})}$ \\
\hline$\alpha$ & $1 s$ & -378.6802 & 0.0033 & 0.0000 & 0.0000 & 0.0000 \\
\hline$\alpha$ & $2 s$ & -47.5198 & 0.0623 & 0.0000 & 0.0000 & 0.0000 \\
\hline$\alpha$ & $2 p_{z}$ & -42.4832 & 0.0452 & -0.0181 & -0.0181 & +0.0362 \\
\hline$\alpha$ & $2 p_{x}$ & -42.4826 & 0.0452 & +0.0362 & -0.0181 & -0.0181 \\
\hline$\alpha$ & $2 p_{y}$ & -42.4826 & 0.0452 & -0.0181 & +0.0362 & -0.0181 \\
\hline$\alpha$ & $3 s$ & -6.2558 & 0.5350 & +0.0004 & +0.0004 & -0.0008 \\
\hline$\alpha$ & $3 p_{x}$ & -4.4690 & 0.5485 & +0.4388 & -0.2194 & -0.2194 \\
\hline$\alpha$ & $3 p_{y}$ & -4.4690 & 0.5485 & -0.2194 & +0.4388 & -0.2194 \\
\hline$\alpha$ & $3 p_{z}$ & -4.4678 & 0.5474 & -0.2189 & -0.2189 & +0.4379 \\
\hline$\alpha$ & $3 d_{x^{2}-y^{2}}$ & -1.1813 & 0.7753 & +0.2215 & +0.2215 & -0.4430 \\
\hline$\alpha$ & $3 d_{x y}$ & -1.1813 & 0.7753 & +0.2215 & +0.2215 & -0.4430 \\
\hline$\alpha$ & $3 d_{y z}$ & -1.1761 & 0.7753 & -0.4430 & +0.2215 & +0.2215 \\
\hline$\alpha$ & $3 d_{z x}$ & -1.1761 & 0.7753 & +0.2215 & -0.4430 & +0.2215 \\
\hline$\alpha$ & $3 d_{z^{2}}$ & -1.1743 & 0.7757 & -0.2239 & -0.2239 & +0.4478 \\
\hline$\alpha$ & $4 s^{z^{-}}$ & -0.4471 & 7.5754 & +0.0036 & +0.0036 & -0.0072 \\
\hline$\alpha$ & $4 p_{z}$ & -0.2086 & 13.9574 & -5.5830 & -5.5830 & +11.1659 \\
\hline$\beta$ & $1 s$ & -378.6792 & 0.0033 & 0.0000 & 0.0000 & 0.0000 \\
\hline$\beta$ & $2 s$ & -47.5192 & 0.0623 & 0.0000 & 0.0000 & 0.0000 \\
\hline$\beta$ & $2 p_{x}$ & -42.4823 & 0.0452 & +0.0362 & -0.0181 & -0.0181 \\
\hline$\beta$ & $2 p_{y}$ & -42.4823 & 0.0452 & -0.0181 & +0.0362 & -0.0181 \\
\hline$\beta$ & $2 p_{z}$ & -42.4796 & 0.0452 & -0.0181 & -0.0181 & +0.0362 \\
\hline$\beta$ & $3 s$ & -6.2535 & 0.5349 & +0.0007 & +0.0007 & -0.0013 \\
\hline$\beta$ & $3 p_{x}$ & -4.4682 & 0.5483 & +0.4386 & -0.2193 & -0.2193 \\
\hline$\beta$ & $3 p_{y}$ & -4.4682 & 0.5483 & -0.2193 & +0.4386 & -0.2193 \\
\hline$\beta$ & $3 p_{z}$ & -4.4585 & 0.5477 & -0.2191 & -0.2191 & +0.4382 \\
\hline$\beta$ & $3 d_{x^{2}-y^{2}}$ & -1.1808 & 0.7753 & +0.2215 & +0.2215 & -0.4430 \\
\hline$\beta$ & $3 d_{x y}$ & -1.1808 & 0.7753 & +0.2215 & +0.2215 & -0.4430 \\
\hline$\beta$ & $3 d_{y z}$ & -1.1720 & 0.7753 & -0.4430 & +0.2215 & +0.2215 \\
\hline$\beta$ & $3 d_{z x}$ & -1.1720 & 0.7753 & +0.2215 & -0.4430 & +0.2215 \\
\hline$\beta$ & $3 d_{z^{2}}$ & -1.1691 & 0.7756 & -0.2133 & -0.2133 & +0.4267 \\
\hline$\beta$ & $4 s$ & -0.3877 & 7.3770 & -0.0070 & -0.0070 & +0.0139 \\
\hline Total & & & 41.4245 & -5.5788 & -5.5788 & +11.1576 \\
\hline
\end{tabular}


[1] Maruhn J A, Reinhard P G and Suraud E 2010 Simple Models of Many-Fermion Systems (Berlin Heidelberg: Springer-Verlag)

[2] Hagino K and Maeno Y 2020 Found. Chem. 22267

[3] Maeno Y, Hagino K and Ishiguro T 2021 Found. Chem. 23201

[4] Bohr A and Mottelson B R 1975 Nuclear Deformations (Nuclear Structure vol 2) (W. A. Benjamin)

[5] Ring P and Schuck P 1980 The Nuclear Many-Body Problem (Berlin Heidelberg: SpringerVerlag)

[6] Federman P and Pittel S 1977 Phys. Lett. B 69385

[7] Federman P, Pittel S and Campos R 1979 Phys. Lett. B 829

[8] Federman P and Pittel S 1979 Phys. Rev. C 20820

[9] Dobaczewski J, Nazarewicz W, Skalski J and Werner T 1988 Phys. Rev. Lett. 602254

[10] Arima A and Horie H 1954 Prog. Theor. Phys. 11509

[11] Minamisono T, Fukuda S, Ohtsubo T, Kitagawa A, Nakayama Y, Someda Y, Takeda S, Fukuda M, Matsuta K and Nojiri Y 1993 Nucl. Phys. A 559239

[12] Minamisono T, Matsuta K, Minamisono K, Kudo S, Ogura M, Fukuda S, Sato K, Mihara M and Fukuda M 2001 Hyperfine Interact. 136225

[13] Ohtsubo T, Stone N J, Stone J R, Towner I S, Bingham C R, Gaulard C, Köster U, Muto S, Nikolov J, Nishimura K, Simpson G S, Soti G, Veskovic M, Walters W B and Wauters F 2012 Phys Rev Lett 109032504

[14] Sagawa H and Alex Brown B 1984 Nucl. Phys. A 43084

[15] Morinaga H 1956 Phys. Rev. 101254

[16] Morinaga H 1966 Phys. Lett. 2178

[17] Ikeda K, Takigawa N and Horiuchi H 1968 Prog. Theor. Phys. Suppl. E68 464

[18] Kanada-En'yo Y, Horiuchi H and Doté A 1999 Phys. Rev. C 60064304

[19] Itagaki N, Okabe S, Ikeda K and Tanihata I 2001 Phys. Rev. C 64014301

[20] von Oertzen W, Freer M and Kanada-En'yo Y 2006 Phys. Rep. 43243

[21] Itoh M, Akimune H, Fujiwara M, Garg U, Hashimoto N, Kawabata T, Kawase K, Kishi S, Murakami T, Nakanishi K, Nakatsugawa Y, Nayak B K, Okumura S, Sakaguchi H, Takeda H, Terashima S, Uchida M, Yasuda Y, Yosoi M and Zenihiro J 2011 Phys. Rev. C 84054308

[22] Freer M and Fynbo H 2014 Prog. Part. Nucl. Phys. 781

[23] Baba T and Kimura M 2016 Phys. Rev. C 94044303

[24] Baba T and Kimura M 2017 Phys. Rev. C 95064318

[25] Itagaki N and Naito T 2021 Phys. Rev. C 103044303

[26] Johnson R C and Rettew R R 1965 J. Chem. Educ. 42145

[27] Cohen I 1965 J. Chem. Educ. 42397

[28] Friedrich H 2006 Theoretical Atomic Physics (Berlin Heidelberg: Springer-Verlag)

[29] Fertig H A and Kohn W 2000 Phys. Rev. A 62052511

[30] Borgoo A, Scharf O, Gaigalas G and Godefroid M 2010 Comput. Phys. Commun. 181426

[31] Austin B J, Heine V and Sham L J 1962 Phys. Rev. 127276

[32] Louie S G, Froyen S and Cohen M L 1982 Phys. Rev. B 261738

[33] Martin R M 2004 Electronic Structure (Cambridge: Cambridge University Press)

[34] Schwerdtfeger P 2011 ChemPhysChem 123143

[35] Roothaan C C J 1951 Rev. Mod. Phys. 2369

[36] Pople J A and Nesbet R K 1954 J. Chem. Phys. 22571

[37] Berthier G 1954 C. R. Acad. Sci. 23891

[38] McWeeny R and Diercksen G 1968 J. Chem. Phys. 494852

[39] Jensen F 2017 Introduction to Computational Chemistry 3rd ed (Chichester: John Wiley \& Sons)

[40] Møller C and Plesset M S 1934 Phys. Rev. 46618

[41] Coester F 1958 Nucl. Phys. 7421

[42] Čížek J 1966 J. Chem. Phys. 454256

[43] Čížek J and Paldus J 1971 Int. J. Quantum Chem. 5359

[44] Pople J A, Binkley J S and Seeger R 1976 Int. J. Quantum Chem. 101

[45] Pople J A, Seeger R and Krishnan R 1977 Int. J. Quantum Chem. 12149

[46] Raghavachari K and Pople J A 1978 Int. J. Quantum Chem. 1491

[47] Krishnan R, Schlegel H B and Pople J A 1980 J. Chem. Phys. 724654

[48] Trucks G W, Salter E, Sosa C and Bartlett R J 1988 Chem. Phys. Lett. 147359

[49] Head-Gordon M, Pople J A and Frisch M J 1988 Chem. Phys. Lett. 153503

[50] Raghavachari K, Pople J A, Replogle E S and Head-Gordon M 1990 J. Phys. Chem. 945579

[51] Pople J A 1999 Rev. Mod. Phys. 711267 
[52] Hohenberg P and Kohn W 1964 Phys. Rev. 136 B864

[53] Kohn W and Sham L J 1965 Phys. Rev. 140 A1133

[54] Kohn W 1999 Rev. Mod. Phys. 711253

[55] Nilsson S G 1955 Dan. Mat. Fys. Medd. 291

[56] Mottelson B R and Nilsson S G 1955 Phys. Rev. 991615

[57] Ragnarsson I and Nilsson S G 2005 Shapes and Shells in Nuclear Structure (Cambridge: Cambridge University Press)

[58] Blanco M A, Flórez M and Bermejo M 1997 J. Mol. Struct. 41919

[59] Frisch M J, Trucks G W, Schlegel H B, Scuseria G E, Robb M A, Cheeseman J R, Scalmani G, Barone V, Petersson G A, Nakatsuji H, Li X, Caricato M, Marenich A V, Bloino J, Janesko B G, Gomperts R, Mennucci B, Hratchian H P, Ortiz J V, Izmaylov A F, Sonnenberg J L, Williams-Young D, Ding F, Lipparini F, Egidi F, Goings J, Peng B, Petrone A, Henderson T, Ranasinghe D, Zakrzewski V G, Gao J, Rega N, Zheng G, Liang W, Hada M, Ehara M, Toyota K, Fukuda R, Hasegawa J, Ishida M, Nakajima T, Honda Y, Kitao O, Nakai H, Vreven T, Throssell K, Montgomery Jr J A, Peralta J E, Ogliaro F, Bearpark M J, Heyd J J, Brothers E N, Kudin K N, Staroverov V N, Keith T A, Kobayashi R, Normand J, Raghavachari K, Rendell A P, Burant J C, Iyengar S S, Tomasi J, Cossi M, Millam J M, Klene M, Adamo C, Cammi R, Ochterski J W, Martin R L, Morokuma K, Farkas O, Foresman J B and Fox D J 2016 Gaussian 16 Revision A.03 Gaussian Inc. Wallingford CT

[60] Mohr P J, Newell D B and Taylor B N 2016 Rev. Mod. Phys. 88035009

[61] Tiesinga E, Mohr P J, Newell D B and Taylor B N 2019 The 2018 CODATA Recommended Values of the Fundamental Physical Constants

[62] Perdew J P and Zunger A 1981 Phys. Rev. B 235048

[63] Hehre W J, Ditchfield R and Pople J A 1972 J. Chem. Phys. 562257

[64] Clark T, Chandrasekhar J, Spitznagel G W and Schleyer P V R 1983 J. Comput. Chem. 4294

[65] Woon D E and Dunning T H 1993 J. Chem. Phys. 981358

[66] Peterson K A, Woon D E and Dunning T H 1994 J. Chem. Phys. 1007410

[67] Hehre W J, Stewart R F and Pople J A 1969 J. Chem. Phys. 512657

[68] Kramida A, Yu Ralchenko, Reader J and and NIST ASD Team 2019 NIST Atomic Spectra Database (ver. 5.7.1), [Online]. Available: https://physics.nist.gov/asd [2020, July 30]. National Institute of Standards and Technology, Gaithersburg, MD.

[69] Foster J P and Weinhold F $1980 \mathrm{~J}$. Am. Chem. Soc. 1027211

[70] Carpenter J E and Weinhold F 1988 J. Mol. Struct. 16941

[71] Reed A E, Curtiss L A and Weinhold F 1988 Chem. Rev. 88899

[72] Glendening E D, Reed A E, Carpenter J E and Weinhold F NBO Version 3.1 Gaussian Inc. Wallingford CT

[73] Cotton F A, Wilkinson G and Gauss P L 1995 Basic Inorganic Chemistry (Chichester: John Wiley \& Sons)

[74] Hund F 1925 Z. Phys. 34296

[75] Hund F 1925 Z. Phys. 33345

[76] Slater J C 1929 Phys. Rev. 341293

[77] Hongo K, Maezono R, Kawazoe Y, Yasuhara H, Towler M D and Needs R J 2004 J. Chem. Phys. 1217144

[78] Oyamada T, Hongo K, Kawazoe Y and Yasuhara H 2010 J. Chem. Phys. 133164113

[79] Schiff L 1968 Quantum Mechanics 3rd ed (New York: McGraw-Hill)

[80] Parr R G and Weitao Y 1989 Density-Functional Theory of Atoms and Molecules (New York: Oxford University Press)

[81] Serot B D and Walecka J D 1986 The Relativistic Nuclear Many-Body Problem Advances in Nuclear Physics vol 16 ed Negele J and Vogt E (Plenum Press)

[82] Bohigas O, Lane A and Martorell J 1979 Phys. Rep. 51267

[83] Bertsch G F and Feldmeier H 1997 Phys. Rev. C 56839

[84] Yukawa H 1935 Proc. Phys. Math. Soc. Jpn. Third 1748

[85] Stoks V G J, Klomp R A M, Terheggen C P F and de Swart J J 1994 Phys. Rev. C 492950

[86] Wiringa R B, Stoks V G J and Schiavilla R 1995 Phys. Rev. C 5138

[87] Machleidt R 2001 Phys. Rev. C 63024001

[88] Ishii N, Aoki S and Hatsuda T 2007 Phys. Rev. Lett. 99022001

[89] Krane K S 1988 Introductory Nuclear Physics (Chichester: John Wiley \& Sons)

[90] Povh B, Rith K, Scholz C and Zetsche F 2008 Particles and Nuclei (Berlin Heidelberg: SpringerVerlag)

[91] Bender M, Heenen P H and Reinhard P G 2003 Rev. Mod. Phys. 75121

[92] Bohr N and Wheeler J A 1939 Phys. Rev. 56426 
[93] Povh B, Rith K, Scholz C, Zetsche F and Rodejohann W 2015 Particles and Nuclei (Berlin Heidelberg: Springer-Verlag)

[94] Engel E and Dreizler R M 2011 Density Functional Theory-An Advanced Course Theoretical and Mathematical Physics (Berlin, Heidelberg: Springer-Verlag)

[95] Wigner E 1934 Phys. Rev. 461002

[96] Vautherin D and Brink D M 1972 Phys. Rev. C 5626

[97] Vautherin D 1973 Phys. Rev. C 7296

[98] Gallagher T F 2005 Rydberg atoms vol 3 (Cambridge: Cambridge University Press)

[99] Saffman M, Walker T G and Mølmer K 2010 Rev. Mod. Phys. 822313

[100] Luukko P J J and Rost J M 2017 Phys. Rev. Lett. 119203001

[101] Stodolna A S, Rouzée A, Lépine F, Cohen S, Robicheaux F, Gijsbertsen A, Jungmann J H, Bordas C and Vrakking M J J 2013 Phys. Rev. Lett. 110213001

[102] Cohen S, Harb M M, Ollagnier A, Robicheaux F, Vrakking M J J, Barillot T, Lépine F and Bordas C 2016 Phys. Rev. A 94013414

[103] Itatani J, Levesque J, Zeidler D, Niikura H, Pépin H, Kieffer J C, Corkum P B and Villeneuve D M 2004 Nature 432867

[104] Pudliner B S, Smerzi A, Carlson J, Pandharipande V R, Pieper S C and Ravenhall D G 1996 Phys. Rev. Lett. 762416

[105] Smerzi A, Ravenhall D G and Pandharipande V R 1997 Phys. Rev. C 562549

[106] Gandolfi S, Carlson J and Pieper S C 2011 Phys. Rev. Lett. 106012501

[107] Bogner S K, Furnstahl R J, Hergert H, Kortelainen M, Maris P, Stoitsov M and Vary J P 2011 Phys. Rev. C 84044306

[108] Maris P, Vary J P, Gandolfi S, Carlson J and Pieper S C 2013 Phys. Rev. C 87054318

[109] Shen S, Liang H, Meng J, Ring P and Zhang S 2018 Phys. Rev. C 97054312

[110] Shen S, Colò G and Roca-Maza X 2019 Phys. Rev. C 99034322

[111] Wang Z, Naito T, Liang H and Long W H 2021 Chin. Phys. C 45064103

[112] Zhao P W and Gandolfi S 2016 Phys. Rev. C 94041302

[113] Zyla P A, Barnett R M, Beringer J, Dahl O, Dwyer D A, Groom D E, Lin C J, Lugovsky K S, Pianori E, Robinson D J, Wohl C G, Yao W M, Agashe K, Aielli G, Allanach B C, Amsler C, Antonelli M, Aschenauer E C, Asner D M, Baer H, Banerjee S, Baudis L, Bauer C W, Beatty J J, Belousov V I, Bethke S, Bettini A, Biebel O, Black K M, Blucher E, Buchmuller O, Burkert V, Bychkov M A, Cahn R N, Carena M, Ceccucci A, Cerri A, Chakraborty D, Chivukula R S, Cowan G, D'Ambrosio G, Damour T, de Florian D, de Gouvêa A, DeGrand T, de Jong P, Dissertori G, Dobrescu B A, D'Onofrio M, Doser M, Drees M, Dreiner H K, Eerola P, Egede U, Eidelman S, Ellis J, Erler J, Ezhela V V, Fetscher W, Fields B D, Foster B, Freitas A, Gallagher H, Garren L, Gerber H J, Gerbier G, Gershon T, Gershtein Y, Gherghetta T, Godizov A A, Gonzalez-Garcia M C, Goodman M, Grab C, Gritsan A V, Grojean C, Grünewald M, Gurtu A, Gutsche T, Haber H E, Hanhart C, Hashimoto S, Hayato Y, Hebecker A, Heinemeyer S, Heltsley B, Hernández-Rey J J, Hikasa K, Hisano J, Höcker A, Holder J, Holtkamp A, Huston J, Hyodo T, Johnson K F, Kado M, Karliner M, Katz U F, Kenzie M, Khoze V A, Klein S R, Klempt E, Kowalewski R V, Krauss F, Kreps M, Krusche B, Kwon Y, Lahav O, Laiho J, Lellouch L P, Lesgourgues J, Liddle A R, Ligeti Z, Lippmann C, Liss T M, Littenberg L, Lourengo C, Lugovsky S B, Lusiani A, Makida Y, Maltoni F, Mannel T, Manohar A V, Marciano W J, Masoni A, Matthews J, Meißner U G, Mikhasenko M, Miller D J, Milstead D, Mitchell R E, Mönig K, Molaro P, Moortgat F, Moskovic M, Nakamura K, Narain M, Nason P, Navas S, Neubert M, Nevski P, Nir Y, Olive K A, Patrignani C, Peacock J A, Petcov S T, Petrov V A, Pich A, Piepke A, Pomarol A, Profumo S, Quadt A, Rabbertz K, Rademacker J, Raffelt G, Ramani H, Ramsey-Musolf M, Ratcliff B N, Richardson P, Ringwald A, Roesler S, Rolli S, Romaniouk A, Rosenberg L J, Rosner J L, Rybka G, Ryskin M, Ryutin R A, Sakai Y, Salam G P, Sarkar S, Sauli F, Schneider O, Scholberg K, Schwartz A J, Schwiening J, Scott D, Sharma V, Sharpe S R, Shutt T, Silari M, Sjöstrand T, Skands P, Skwarnicki T, Smoot G F, Soffer A, Sozzi M S, Spanier S, Spiering C, Stahl A, Stone S L, Sumino Y, Sumiyoshi T, Syphers M J, Takahashi F, Tanabashi M, Tanaka J, Taševský M, Terashi K, Terning J, Thoma U, Thorne R S, Tiator L, Titov M, Tkachenko N P, Tovey D R, Trabelsi K, Urquijo P, Valencia G, Van de Water R, Varelas N, Venanzoni G, Verde L, Vincter M G, Vogel P, Vogelsang W, Vogt A, Vorobyev V, Wakely S P, Walkowiak W, Walter C W, Wands D, Wascko M O, Weinberg D H, Weinberg E J, White M, Wiencke L R, Willocq S, Woody C L, Workman R L, Yokoyama M, Yoshida R, Zanderighi G, Zeller G P, Zenin O V, Zhu R Y, Zhu S L, Zimmermann F, Anderson J, Basaglia T, Lugovsky V S, Schaffner P and Zheng W (Particle Data Group) 2020 Prog. Theor. Exp. Phys. $2020083 \mathrm{C} 01$ 
[114] Bartel J, Quentin P, Brack M, Guet C and Håkansson H B 1982 Nucl. Phys. A 38679 